\title{
Iterative Algorithms for Systems of Generalized Equilibrium Problems with the Constraints of Variational Inclusion and Fixed Point Problems
}

\author{
Lu-Chuan Ceng, ${ }^{1}$ Abdul Latif, ${ }^{2}$ and Abdullah E. Al-Mazrooei ${ }^{2}$ \\ ${ }^{1}$ Department of Mathematics, Shanghai Normal University, and Scientific Computing Key Laboratory of Shanghai Universities, \\ Shanghai 200234, China \\ ${ }^{2}$ Department of Mathematics, King Abdulaziz University, P.O. Box 80203, Jeddah 21589, Saudi Arabia
}

Correspondence should be addressed to Abdul Latif; alatif@kau.edu.sa

Received 6 December 2013; Accepted 11 January 2014; Published 12 March 2014

Academic Editor: Jen-Chih Yao

Copyright (C) 2014 Lu-Chuan Ceng et al. This is an open access article distributed under the Creative Commons Attribution License, which permits unrestricted use, distribution, and reproduction in any medium, provided the original work is properly cited.

We introduce and analyze a hybrid extragradient-like viscosity iterative algorithm for finding a common solution of a systems of generalized equilibrium problems and a generalized mixed equilibrium problem with the constraints of two problems: a finite family of variational inclusions for maximal monotone and inverse strongly monotone mappings and a fixed point problem of infinitely many nonexpansive mappings in a real Hilbert space. Under some suitable conditions, we prove the strong convergence of the sequence generated by the proposed algorithm to a common solution of these problems.

\section{Introduction}

Let $H$ be a real Hilbert space with inner product $\langle\cdot, \cdot\rangle$ and norm $\|\cdot\|, C$ be a nonempty closed convex subset of $H$ and $P_{C}$ be the metric projection of $H$ onto $C$. Let $S: C \rightarrow H$ be a nonlinear mapping on $C$. We denote by $\operatorname{Fix}(S)$ the set of fixed points of $S$ and by $\mathbf{R}$ the set of all real numbers. A mapping $V$ is called strongly positive on $H$ if there exists a constant $\bar{\gamma} \in(0,1]$ such that

$$
\langle V x, x\rangle \geq \bar{\gamma}\|x\|^{2}, \quad \forall x \in H .
$$

A mapping $S: C \rightarrow H$ is called $L$-Lipschitz continuous if there exists a constant $L \geq 0$ such that

$$
\|S x-S y\| \leq L\|x-y\|, \quad \forall x, y \in C .
$$

In particular, if $L=1$ then $S$ is called a nonexpansive mapping; if $L \in[0,1)$ then $A$ is called a contraction.

Let $A: C \rightarrow H$ be a nonlinear mapping on $C$. We consider the following variational inequality problem (VIP) [1] which is to find a point $x \in C$ such that

$$
\langle A x, y-x\rangle \geq 0, \quad \forall y \in C .
$$

The solution set of $\operatorname{VIP}(3)$ is denoted by $\operatorname{VI}(C, A)$.
Let $\varphi: C \rightarrow \mathbf{R}$ be a real-valued function, $A: H \rightarrow H$ be a nonlinear mapping and $\Theta: C \times C \rightarrow \mathbf{R}$ be a bifunction. In 2008, Peng and Yao [2] introduced the following generalized mixed equilibrium problem (GMEP) of finding $x \in C$ such that

$$
\Theta(x, y)+\varphi(y)-\varphi(x)+\langle A x, y-x\rangle \geq 0, \quad \forall y \in C .
$$

We denote the set of solutions of GMEP (4) by $\operatorname{GMEP}(\Theta, \varphi, A)$. The system of equilibrium problems or generalized equilibrium problems is a tool to study Nash eequilibrium problems, see for example [3-8]. In fact, the GMEP (4) is very general in the sense that it includes, as special cases, optimization problems, variational inequalities, minimax problems, Nash equilibrium problems in noncooperative games and others. The GMEP is further considered and studied; see for example, [9-15]. Here we also consider a system of two generalized equilibrium problem that could be usefull to study the Two players game problem, see [16].

Throughout this paper, it is assumed as in [2] that $\Theta: C \times$ $C \rightarrow \mathbf{R}$ is a bifunction satisfying conditions (H1)-(H4) and 
$\varphi: C \rightarrow \mathbf{R}$ is a lower semicontinuous and convex function with restriction (H5), where

(H1) $\Theta(x, x)=0$ for all $x \in C$;

$(\mathrm{H} 2) \Theta$ is monotone, that is, $\Theta(x, y)+\Theta(y, x) \leq 0$ for any $x, y \in C$;

(H3) $\Theta$ is upper-hemicontinuous, that is, for each $x, y, z \in$ C,

$$
\limsup _{t \rightarrow 0^{+}} \Theta(t z+(1-t) x, y) \leq \Theta(x, y) ;
$$

(H4) $\Theta(x, \cdot)$ is convex and lower semicontinuous for each $x \in C$;

(H5) for each $x \in H$ and $r>0$, there exists a bounded subset $D_{x} \subset C$ and $y_{x} \in C$ such that for any $z \in C \backslash D_{x}$,

$$
\Theta\left(z, y_{x}\right)+\varphi\left(y_{x}\right)-\varphi(z)+\frac{1}{r}\left\langle y_{x}-z, z-x\right\rangle<0
$$

Given a positive number $r>0$. Let $S_{r}^{(\Theta, \varphi)}: H \rightarrow C$ is the solution set of the auxiliary mixed equilibrium problem, that is, for each $x \in H$,

$$
\begin{aligned}
S_{r}^{(\Theta, \varphi)}(x):=\{y \in C: \Theta(y, z)+\varphi(z)-\varphi(y) \\
\left.\quad+\frac{1}{r}\left\langle K^{\prime}(y)-K^{\prime}(x), z-y\right\rangle \geq 0, \forall z \in C\right\} .
\end{aligned}
$$

In particular, whenever $K(x)=(1 / 2)\|x\|^{2}, \forall x \in H, S_{r}^{(\Theta, \varphi)}$ is rewritten as $T_{r}^{(\Theta, \varphi)}$.

Let $\Theta_{1}, \Theta_{2}: C \times C \rightarrow \mathbf{R}$ be two bifunctions, and $A_{1}, A_{2}: C \rightarrow H$ be two nonlinear mappings. Consider the following system of generalized equilibrium problems (SGEP): find $\left(x^{*}, y^{*}\right) \in C \times C$ such that

$$
\begin{array}{r}
\Theta_{1}\left(x^{*}, x\right)+\left\langle A_{1} y^{*}, x-x^{*}\right\rangle+\frac{1}{v_{1}}\left\langle x^{*}-y^{*}, x-x^{*}\right\rangle \geq 0, \\
\forall x \in C, \\
\Theta_{2}\left(y^{*}, y\right)+\left\langle A_{2} x^{*}, y-y^{*}\right\rangle+\frac{1}{v_{2}}\left\langle y^{*}-x^{*}, y-y^{*}\right\rangle \geq 0, \\
\forall y \in C,
\end{array}
$$

where $v_{1}>0$ and $v_{2}>0$ are two constants. It is introduced and studied in [17]. Whenever $\Theta_{1} \equiv \Theta_{2} \equiv 0$, the SGEP reduces to a system of variational inequalities, which is considered and studied in [18]. It is worth to mention that the system of variational inequalities is a tool to solve the Nash equilibrium problem for noncooperative games.

In 2010, Ceng and Yao [17] transformed the SGEP into a fixed point problem in the following way.

Proposition CY (see [17]). Let $\Theta_{1}, \Theta_{2}: C \times C \rightarrow \mathbf{R}$ be two bifunctions satisfying conditions $(\mathrm{H1})-(\mathrm{H} 4)$ and let $A_{k}$ :
$C \rightarrow H$ be $\zeta_{k}$-inverse-strongly monotone for $k=1,2$. Let $\nu_{k} \in\left(0,2 \zeta_{k}\right)$ for $k=1,2$. Then,$\left(x^{*}, y^{*}\right) \in C \times C$ is a solution of SGEP (8) if and only if $x^{*}$ is a fixed point of the mapping $G: C \rightarrow C$ defined by $G=T_{\nu_{1}}^{\Theta_{1}}\left(I-\nu_{1} A_{1}\right) T_{\nu_{2}}^{\Theta_{2}}\left(I-\nu_{2} A_{2}\right)$ where $y^{*}=T_{v_{2}}^{\Theta_{2}}\left(I-v_{2} A_{2}\right) x^{*}$. Here, we denote the fixed point set of $G$ by $\operatorname{SGEP}(G)$.

Let $\left\{T_{n}\right\}_{n=1}^{\infty}$ be an infinite family of nonexpansive mappings on $H$ and $\left\{\lambda_{n}\right\}_{n=1}^{\infty}$ be a sequence of nonnegative numbers in $[0,1]$. For any $n \geq 1$, define a mapping $W_{n}$ on $H$ as follows:

$$
\begin{gathered}
U_{n, n+1}=I, \\
U_{n, n}=\lambda_{n} T_{n} U_{n, n+1}+\left(1-\lambda_{n}\right) I, \\
U_{n, n-1}=\lambda_{n-1} T_{n-1} U_{n, n}+\left(1-\lambda_{n-1}\right) I, \\
\ldots \\
U_{n, k}=\lambda_{k} T_{k} U_{n, k+1}+\left(1-\lambda_{k}\right) I, \\
U_{n, k-1}=\lambda_{k-1} T_{k-1} U_{n, k}+\left(1-\lambda_{k-1}\right) I, \\
\ldots \\
U_{n, 2}=\lambda_{2} T_{2} U_{n, 3}+\left(1-\lambda_{2}\right) I, \\
W_{n}=U_{n, 1}=\lambda_{1} T_{1} U_{n, 2}+\left(1-\lambda_{1}\right) I .
\end{gathered}
$$

Such a mapping $W_{n}$ is called the $W$-mapping generated by $T_{n}, T_{n-1}, \ldots, T_{1}$ and $\lambda_{n}, \lambda_{n-1}, \ldots, \lambda_{1}$.

In 2011, for the case where $C=H$, Yao et al. [14] proposed the following hybrid iterative algorithm

$$
\begin{gathered}
\Theta\left(y_{n}, z\right)+\varphi(z)-\varphi\left(y_{n}\right) \\
\quad+\frac{1}{r}\left\langle K^{\prime}\left(y_{n}\right)-K^{\prime}\left(x_{n}\right), z-y_{n}\right\rangle \geq 0, \quad z \in H, \\
x_{n+1}=\alpha_{n}\left(u+\gamma f\left(x_{n}\right)\right)+\beta_{n} x_{n} \\
+\left(\left(1-\beta_{n}\right) I-\alpha_{n}(I+\mu V)\right) W_{n} y_{n}, \quad \forall n \geq 1,
\end{gathered}
$$

where $f: H \rightarrow H$ be a contraction, $K: H \rightarrow \mathbf{R}$ is differentiable and strongly convex, $\left\{\alpha_{n}\right\},\left\{\beta_{n}\right\} \subset(0,1)$ and $x_{0}, u \in H$ are given, for finding a common element of the set $\operatorname{MEP}(\Theta, \varphi)$ and the fixed point set $\cap_{n=1}^{\infty} \operatorname{Fix}\left(T_{n}\right)$ of an infinite family of nonexpansive mappings $\left\{T_{n}\right\}_{n=1}^{\infty}$ on $H$. They proved the strong convergence of the sequence generated by the hybrid iterative algorithm (10) to a point $x^{*} \in \cap_{n=1}^{\infty} \operatorname{Fix}\left(T_{n}\right) \cap$ $\operatorname{MEP}(\Theta, \varphi)$ under some appropriate conditions. This point $x^{*}$ also solves the following optimization problem:

$$
\min _{x \in \cap_{n=1}^{\infty}} \underset{\operatorname{Fix}\left(T_{n}\right) \cap \operatorname{MEP}(\Theta, \varphi)}{ } \frac{\mu}{2}\langle V x, x\rangle+\frac{1}{2}\|x-u\|^{2}-h(x),
$$

where $h: H \rightarrow \mathbf{R}$ is the potential function of $\gamma f$. 
Let $f: H \rightarrow H$ be a contraction and $V$ be a strongly positive bounded linear operator on $H$. Assume that $\varphi$ : $H \rightarrow \mathbf{R}$ is a lower semicontinuous and convex functional, that $\Theta, \Theta_{1}, \Theta_{2}: H \times H \rightarrow \mathbf{R}$ satisfy conditions (H1)(H4), and that $A, A_{1}, A_{2}: H \rightarrow H$ are inverse-strongly monotone. Let the mapping $G$ be defined as in Proposition CY. Very recently, Ceng et al. [11] introduced the following hybrid extragradient-like iterative algorithm

$$
\begin{gathered}
z_{n}=S_{r_{n}}^{(\Theta, \varphi)}\left(x_{n}-r_{n} A x_{n}\right), \\
x_{n+1}=\alpha_{n}\left(u+\gamma f\left(x_{n}\right)\right)+\beta_{n} x_{n} \\
+\left(\left(1-\beta_{n}\right) I-\alpha_{n}(I+\mu V)\right) W_{n} G z_{n}, \quad \forall n \geq 0,
\end{gathered}
$$

for finding a common solution of GMEP (4), SGEP (8) and the fixed point problem of an infinite family of nonexpansive mappings $\left\{T_{n}\right\}_{n=1}^{\infty}$ on $H$, where $\left\{r_{n}\right\} \subset(0, \infty),\left\{\alpha_{n}\right\},\left\{\beta_{n}\right\} \subset$ $(0,1), v_{k} \in\left(0,2 \zeta_{k}\right), k=1,2$, and $x_{0}, u \in H$ are given. The authors proved the strong convergence of the sequence generated by the hybrid iterative algorithm (11) to a point $x^{*} \in \cap_{n=1}^{\infty} \operatorname{Fix}\left(T_{n}\right) \cap \operatorname{GMEP}(\Theta, \varphi, A) \cap \operatorname{SGEP}(G)$ under some suitable conditions. This point $x^{*}$ also solves the following optimization problem:

$$
\begin{gathered}
\min _{x \in \cap_{n=1}^{\infty} \operatorname{Fix}\left(T_{n}\right) \cap G M E P(\Theta, \varphi, A) \cap S G E P(G)} \frac{\mu}{2}\langle V x, x\rangle \\
+\frac{1}{2}\|x-u\|^{2}-h(x),
\end{gathered}
$$

where $h: H \rightarrow \mathbf{R}$ is the potential function of $\gamma f$.

On the other hand, let $B$ be a single-valued mapping of $C$ into $H$ and $R$ be a set-valued mapping with $D(R)=C$. Consider the following variational inclusion: find a point $x \in$ $C$ such that

$$
0 \in B x+R x .
$$

We denote by $I(B, R)$ the solution set of the variational inclusion (12). In particular, if $B=R=0$, then $I(B, R)=$ $C$. If $B=0$, then problem (12) becomes the inclusion problem introduced by Rockafellar [19]. It is known that problem (12) provides a convenient framework for the unified study of optimal solutions in many optimization related areas including mathematical programming, complementarity problems, variational inequalities, optimal control, mathematical economics, equilibria and game theory, and so forth. Let a set-valued mapping $R: D(R) \subset H \rightarrow 2^{H}$ be maximal monotone. We define the resolvent operator $J_{R, \lambda}$ : $H \rightarrow \overline{D(R)}$ associated with $R$ and $\lambda$ as follows:

$$
J_{R, \lambda}=(I+\lambda R)^{-1}, \quad \forall x \in H,
$$

where $\lambda$ is a positive number.

In this paper, we will introduce and analyze an iterative algorithm by hybrid extragradient-like viscosity method for finding a common solution of a systems of generalized equilibrium problems and a generalized mixed equilibrium problem with the constraints of two problems: a finite family of variational inclusions for maximal monotone and inverse strongly monotone mappings and a fixed point problem of infinitely many nonexpansive mappings in a real Hilbert space. Under some suitable conditions, we prove the strong convergence of the sequence generated by the proposed algorithm to a common solution of these problems. Such solution also solves an optimization problem. Several special cases are also discussed. The results presented in this paper are the supplement, extension, improvement and generalization of the previously known results in this area.

\section{Preliminaries}

Throughout this paper, we assume that $H$ is a real Hilbert space whose inner product and norm are denoted by $\langle\cdot, \cdot\rangle$ and $\|\cdot\|$, respectively. Let $C$ be a nonempty closed convex subset of $H$. We write $x_{n} \rightarrow x$ to indicate that the sequence $\left\{x_{n}\right\}$ converges weakly to $x$ and $x_{n} \rightarrow x$ to indicate that the sequence $\left\{x_{n}\right\}$ converges strongly to $x$. Moreover, we use $\omega_{w}\left(x_{n}\right)$ to denote the weak $\omega$-limit set of the sequence $\left\{x_{n}\right\}$, that is,

$$
\begin{aligned}
\omega_{w}\left(x_{n}\right):= & \left\{x \in H: x_{n_{i}}-x\right. \\
& \text { for some subsequence } \left.\left\{x_{n_{i}}\right\} \text { of }\left\{x_{n}\right\}\right\} .
\end{aligned}
$$

Definition 1. A mapping $A: C \rightarrow H$ is called

(i) monotone if

$$
\langle A x-A y, x-y\rangle \geq 0, \quad \forall x, y \in C,
$$

(ii) $\eta$-strongly monotone if there exists a constant $\eta>0$ such that

$$
\langle A x-A y, x-y\rangle \geq \eta\|x-y\|^{2}, \quad \forall x, y \in C,
$$

(iii) $\zeta$-inverse-strongly monotone if there exists a constant $\zeta>0$ such that

$$
\langle A x-A y, x-y\rangle \geq \zeta\|A x-A y\|^{2}, \quad \forall x, y \in C .
$$

It is easy to see that the projection $P_{C}$ is 1 -ism. Inverse strongly monotone (also referred to as co-coercive) operators have been applied widely in solving practical problems in various fields.

Definition 2. A differentiable function $K: H \rightarrow \mathbf{R}$ is called: (i) convex, if

$$
K(y)-K(x) \geq\left\langle K^{\prime}(x), y-x\right\rangle, \quad \forall x, y \in H,
$$

where $K^{\prime}(x)$ is the Frechet derivative of $K$ at $x$;

(ii) strongly convex, if there exists a constant $\sigma>0$ such that

$$
K(y)-K(x)-\left\langle K^{\prime}(x), y-x\right\rangle \geq \frac{\sigma}{2}\|x-y\|^{2}, \quad \forall x, y \in H .
$$

It is easy to see that if $K: H \rightarrow \mathbf{R}$ is a differentiable strongly convex function with constant $\sigma>0$ then $K^{\prime}: H \rightarrow$ $H$ is strongly monotone with constant $\sigma>0$. 
The metric (or nearest point) projection from $H$ onto $C$ is the mapping $P_{C}: H \rightarrow C$ which assigns to each point $x \in H$ the unique point $P_{C} x \in C$ satisfying the property

$$
\left\|x-P_{C} x\right\|=\inf _{y \in C}\|x-y\|=: d(x, C) .
$$

Some important properties of projections are gathered in the following proposition.

Proposition 3. For given $x \in H$ and $z \in C$ :

(i) $z=P_{C} x \Leftrightarrow\langle x-z, y-z\rangle \leq 0, \forall y \in C$;

(ii) $z=P_{C} x \Leftrightarrow\|x-z\|^{2} \leq\|x-y\|^{2}-\|y-z\|^{2}, \forall y \in C$;

(iii) $\left\langle P_{C} x-P_{C} y, x-y\right\rangle \geq\left\|P_{C} x-P_{C} y\right\|^{2}, \forall y \in H$. (This implies that $P_{C}$ is nonexpansive and monotone.)

By using the technique of [20], we can readily obtain the following elementary result.

Proposition 4 (see [11, Lemma 1 and Proposition 1]). Let $C$ be a nonempty closed convex subset of a real Hilbert space $H$ and let $\varphi: C \rightarrow \mathbf{R}$ be a lower semicontinuous and convex function. Let $\Theta: C \times C \rightarrow \mathbf{R}$ be a bifunction satisfying the conditions (H1)-(H4). Assume that

(i) $K: H \rightarrow \mathbf{R}$ is strongly convex with constant $\sigma>0$ and the function $x \mapsto\left\langle y-x, K^{\prime}(x)\right\rangle$ is weakly upper semicontinuous for each $y \in H$;

(ii) for each $x \in H$ and $r>0$, there exists a bounded subset $D_{x} \subset C$ and $y_{x} \in C$ such that for any $z \in C \backslash D_{x}$,

$\Theta\left(z, y_{x}\right)+\varphi\left(y_{x}\right)-\varphi(z)+\frac{1}{r}\left\langle K^{\prime}(z)-K^{\prime}(x), y_{x}-z\right\rangle<0$.

Then the following hold:

(a) for each $x \in H, S_{r}^{(\Theta, \varphi)}(x) \neq \emptyset$;

(b) $S_{r}^{(\Theta, \varphi)}$ is single-valued;

(c) $S_{r}^{(\Theta, \varphi)}$ is nonexpansive if $K^{\prime}$ is Lipschitz continuous with constant $\nu>0$ and

$$
\begin{aligned}
& \left\langle K^{\prime}\left(x_{1}\right)-K^{\prime}\left(x_{2}\right), u_{1}-u_{2}\right\rangle \\
& \quad \geq\left\langle K^{\prime}\left(u_{1}\right)-K^{\prime}\left(u_{2}\right), u_{1}-u_{2}\right\rangle, \quad \forall\left(x_{1}, x_{2}\right) \in H \times H,
\end{aligned}
$$

where $u_{i}=S_{r}^{(\Theta, \varphi)}\left(x_{i}\right)$ for $i=1,2$;

(d) for all $s, t>0$ and $x \in H$

$$
\begin{aligned}
& \left\langle K^{\prime}\left(S_{s}^{(\Theta, \varphi)} x\right)-K^{\prime}\left(S_{t}^{(\Theta, \varphi)} x\right), S_{s}^{(\Theta, \varphi)} x-S_{t}^{(\Theta, \varphi)} x\right\rangle \\
& \quad \leq \frac{s-t}{s}\left\langle K^{\prime}\left(S_{s}^{(\Theta, \varphi)} x\right)-K^{\prime}(x), S_{s}^{(\Theta, \varphi)} x-S_{t}^{(\Theta, \varphi)} x\right\rangle ;
\end{aligned}
$$

(e) $\operatorname{Fix}\left(S_{r}^{(\Theta, \varphi)}\right)=\operatorname{MEP}(\Theta, \varphi)$;

(f) $\operatorname{MEP}(\Theta, \varphi)$ is closed and convex.
In particular, whenever $\Theta: C \times C \rightarrow \mathbf{R}$ is a bifunction satisfying the conditions (H1)-(H4) and $K(x)=$ $(1 / 2)\|x\|^{2}, \forall x \in H$, then that is, for any $x, y \in H$,

$$
\left\|S_{r}^{(\Theta, \varphi)} x-S_{r}^{(\Theta, \varphi)} y\right\|^{2} \leq\left\langle S_{r}^{(\Theta, \varphi)} x-S_{r}^{(\Theta, \varphi)} y, x-y\right\rangle
$$

$\left(S_{r}^{(\Theta, \varphi)}\right.$ is firmly nonexpansive) and

$$
\begin{array}{r}
\left\|S_{s}^{(\Theta, \varphi)} x-S_{t}^{(\Theta, \varphi)} x\right\| \leq \frac{|s-t|}{s}\left\|S_{s}^{(\Theta, \varphi)} x-x\right\|, \\
\forall s, t>0, \quad x \in H .
\end{array}
$$

In this case, $S_{r}^{(\Theta, \varphi)}$ is rewritten as $T_{r}^{(\Theta, \varphi)}$. If, in addition, $\varphi \equiv 0$, then $T_{r}^{(\Theta, \varphi)}$ is rewritten as $T_{r}^{\Theta}$.

Remark 5. Suppose $K: H \rightarrow \mathbf{R}$ is strongly convex with constant $\sigma>0$ and $K^{\prime}: H \rightarrow H$ is Lipschitz continuous with constant $\nu>0$. Then $K^{\prime}: H \rightarrow H$ is $\sigma$-strongly monotone and $\nu$-Lipschitz continuous with positive constants $\sigma, \nu>0$. Utilizing Proposition 4 (d) we can show that for all $s, t>0$ and $x \in H$,

$$
\left\|S_{s}^{(\Theta, \varphi)} x-S_{t}^{(\Theta, \varphi)} x\right\| \leq \frac{|s-t|}{s} \cdot \frac{v}{\sigma}\left\|S_{s}^{(\Theta, \varphi)} x-x\right\| .
$$

We need some facts and tools in a real Hilbert space $H$ which are listed as lemmas below.

Lemma 6. Let $X$ be a real inner product space. Then there holds the following inequality

$$
\|x+y\|^{2} \leq\|x\|^{2}+2\langle y, x+y\rangle, \quad \forall x, y \in X .
$$

Lemma 7. Let $H$ be a real Hilbert space. Then the following hold:

(a) $\|x-y\|^{2}=\|x\|^{2}-\|y\|^{2}-2\langle x-y, y\rangle$ for all $x, y \in H$;

(b) $\|\lambda x+\mu y\|^{2}=\lambda\|x\|^{2}+\mu\|y\|^{2}-\lambda \mu\|x-y\|^{2}$ for all $x, y \in$ $H$ and $\lambda, \mu \in[0,1]$ with $\lambda+\mu=1$;

(c) If $\left\{x_{n}\right\}$ is a sequence in $H$ such that $x_{n} \rightarrow x$, it follows that

$\limsup _{n \rightarrow \infty}\left\|x_{n}-y\right\|^{2}=\limsup _{n \rightarrow \infty}\left\|x_{n}-x\right\|^{2}+\|x-y\|^{2}, \quad \forall y \in H$.

We have the following crucial lemmas concerning the $W$ mappings defined by (9).

Lemma 8 (see [21, Lemma 3.2]). Let $\left\{T_{n}\right\}_{n=1}^{\infty}$ be a sequence of nonexpansive self-mappings on $H$ such that $\cap_{n=1}^{\infty} \operatorname{Fix}\left(T_{n}\right) \neq \emptyset$ and let $\left\{\lambda_{n}\right\}$ be a sequence in $(0, b]$ for some $b \in(0,1)$. Then, for every $x \in H$ and $k \geq 1$ the limit $\lim _{n \rightarrow \infty} U_{n, k} x$ exists, where $U_{n, k}$ is defined by (9).

Remark 9 (see [22, Remark 3.1]). It can be known from Lemma 8 that if $D$ is a nonempty bounded subset of $H$, then for $\epsilon>0$ there exists $n_{0} \geq k$ such that for all $n>n_{0}$

$$
\sup _{x \in D}\left\|U_{n, k} x-U_{k} x\right\| \leq \epsilon .
$$


Remark 10 (see [22, Remark 3.2]). Utilizing Lemma 8, we define a mapping $W: H \rightarrow H$ as follows:

$$
W x=\lim _{n \rightarrow \infty} W_{n} x=\lim _{n \rightarrow \infty} U_{n, 1} x, \quad \forall x \in H .
$$

Such a $W$ is called the $W$-mapping generated by $T_{1}, T_{2}, \ldots$ and $\lambda_{1}, \lambda_{2}, \ldots$. Since $W_{n}$ is nonexpansive, $W: H \rightarrow H$ is also nonexpansive. Indeed, observe that for each $x, y \in H$

$$
\|W x-W y\|=\lim _{n \rightarrow \infty}\left\|W_{n} x-W_{n} y\right\| \leq\|x-y\| .
$$

If $\left\{x_{n}\right\}$ is a bounded sequence in $H$, then we put $D=\left\{x_{n}\right.$ : $n \geq 1\}$. Hence, it is clear from Remark 5 that for an arbitrary $\epsilon>0$ there exists $N_{0} \geq 1$ such that for all $n>N_{0}$

$$
\left\|W_{n} x_{n}-W x_{n}\right\|=\left\|U_{n, 1} x_{n}-U_{1} x_{n}\right\| \leq \sup _{x \in D}\left\|U_{n, 1} x-U_{1} x\right\| \leq \epsilon .
$$

This implies that

$$
\lim _{n \rightarrow \infty}\left\|W_{n} x_{n}-W x_{n}\right\|=0
$$

Lemma 11 (see [21, Lemma 3.3]). Let $\left\{T_{n}\right\}_{n=1}^{\infty}$ be a sequence of nonexpansive self-mappings on $H$ such that $\cap_{n=1}^{\infty} \operatorname{Fix}\left(T_{n}\right) \neq \emptyset$, and let $\left\{\lambda_{n}\right\}$ be a sequence in $(0, b]$ for some $b \in(0,1)$. Then, $\operatorname{Fix}(W)=\cap_{n=1}^{\infty} \operatorname{Fix}\left(T_{n}\right)$.

Lemma 12 (see [23, Demiclosedness principle]). Let $C$ be a nonempty closed convex subset of a real Hilbert space H. Let $T$ be a nonexpansive self-mapping on $C$. Then $I-T$ is demiclosed. That is, whenever $\left\{x_{n}\right\}$ is a sequence in $C$ weakly converging to some $x \in C$ and the sequence $\left\{(I-T) x_{n}\right\}$ strongly converges to some $y$, it follows that $(I-T) x=y$. Here $I$ is the identity operator of $H$.

Lemma 13. Let $A: C \rightarrow H$ be a monotone mapping. In the context of the variational inequality problem the characterization of the projection (see Proposition 3 (i)) implies

$$
u \in \mathrm{VI}(C, A) \Longleftrightarrow u=P_{C}(u-\lambda A u), \quad \lambda>0 .
$$

Lemma 14 (see [24]). Let $\left\{x_{n}\right\}$ and $\left\{w_{n}\right\}$ be bounded sequences in a real Banach space $X$ and $\left\{\beta_{n}\right\}$ be a sequence in $[0,1]$ with $0<\liminf _{n \rightarrow \infty} \beta_{n} \leq \lim \sup _{n \rightarrow \infty} \beta_{n}<1$. Suppose

$$
\begin{gathered}
x_{n+1}=\beta_{n} x_{n}+\left(1-\beta_{n}\right) w_{n}, \quad \forall n \geq 0, \\
\limsup _{n \rightarrow \infty}\left(\left\|w_{n+1}-w_{n}\right\|-\left\|x_{n+1}-x_{n}\right\|\right) \leq 0 .
\end{gathered}
$$

Then, $\lim _{n \rightarrow \infty}\left\|w_{n}-x_{n}\right\|=0$.

Lemma 15 (see [25]). Assume that $\left\{a_{n}\right\}$ is a sequence of nonnegative real numbers such that

$$
a_{n+1} \leq\left(1-\gamma_{n}\right) a_{n}+\sigma_{n} \gamma_{n}, \quad \forall n \geq 1,
$$

where $\left\{\gamma_{n}\right\}$ is a sequence in $[0,1]$ and $\left\{\sigma_{n}\right\}$ is a real sequence such that

$$
\text { (i) } \sum_{n=1}^{\infty} \gamma_{n}=\infty \text {; }
$$

(ii) $\lim \sup _{n \rightarrow \infty} \sigma_{n} \leq 0$ or $\sum_{n=1}^{\infty}\left|\sigma_{n} \gamma_{n}\right|<\infty$.

Then $\lim _{n \rightarrow \infty} a_{n}=0$.

Recall that a set-valued mapping $T: D(T) \subset H \rightarrow 2^{H}$ is called monotone if for all $x, y \in D(T), f \in T x$ and $g \in T y$ imply

$$
\langle f-g, x-y\rangle \geq 0
$$

A set-valued mapping $T$ is called maximal monotone if $T$ is monotone and $(I+\lambda T) D(T)=H$ for each $\lambda>0$, where $I$ is the identity mapping of $H$. We denote by $G(T)$ the graph of $T$. It is known that a monotone mapping $T$ is maximal if and only if, for $(x, f) \in H \times H,\langle f-g, x-y\rangle \geq 0$ for every $(y, g) \in G(T)$ implies $f \in T x$. Next we provide an example to illustrate the concept of maximal monotone mapping.

Let $A: C \rightarrow H$ be a monotone, $k$-Lipschitz-continuous mapping and let $N_{C} v$ be the normal cone to $C$ at $v \in C$, that is,

$$
N_{C} v=\{w \in H:\langle v-u, w\rangle \geq 0, \forall u \in C\}
$$

Define

$$
T v= \begin{cases}A v+N_{C} v, & \text { if } v \in C, \\ \emptyset, & \text { if } v \notin C .\end{cases}
$$

Then, $T$ is maximal monotone and $0 \in T v$ if and only if $v \in$ $\mathrm{VI}(C, A)$; see [19].

Assume that $R: D(R) \subset H \rightarrow 2^{H}$ is a maximal monotone mapping. Let $\lambda>0$. In terms of Huang [26] (see also [27]), there holds the following property for the resolvent operator $J_{R, \lambda}: H \rightarrow \overline{D(R)}$.

Lemma 16. $J_{R, \lambda}$ is single-valued and firmly nonexpansive, that is,

$$
\left\langle J_{R, \lambda} x-J_{R, \lambda} y, x-y\right\rangle \geq\left\|J_{R, \lambda} x-J_{R, \lambda} y\right\|^{2}, \quad \forall x, y \in H .
$$

Consequently, $J_{R, \lambda}$ is nonexpansive and monotone.

Lemma 17 (see [28]). Let $R$ be a maximal monotone mapping with $D(R)=C$. Then for any given $\lambda>0, u \in C$ is a solution of problem (12) if and only if $u \in C$ satisfies

$$
u=J_{R, \lambda}(u-\lambda B u) .
$$

Lemma 18 (see [27]). Let $R$ be a maximal monotone mapping with $D(R)=C$ and let $B: C \rightarrow H$ be a strongly monotone, continuous and single-valued mapping. Then for each $z \in H$, the equation $z \in(B+\lambda R) x$ has a unique solution $x_{\lambda}$ for $\lambda>0$.

Lemma 19 (see [28]). Let $R$ be a maximal monotone mapping with $D(R)=C$ and $B: C \rightarrow H$ be a monotone, continuous and single-valued mapping. Then $(I+\lambda(R+B)) C=H$ for each $\lambda>0$. In this case, $R+B$ is maximal monotone.

Lemma 20 (see [29]). Let $C$ be a nonempty closed convex subset of a real Hilbert space $H$, and $g: C \rightarrow \mathbf{R} \cup+\infty$ be 
a proper lower semicontinuous differentiable convex function. If $x^{*}$ is a solution the minimization problem

$$
g\left(x^{*}\right)=\inf _{x \in C} g(x)
$$

then,

$$
\left\langle g^{\prime}(x), x-x^{*}\right\rangle \geq 0, \quad \forall x \in C .
$$

In particular, if $x^{*}$ solves $(O P)$, then

$$
\left\langle u+(\gamma f-(I+\mu V)) x^{*}, x-x^{*}\right\rangle \leq 0 .
$$

\section{Main Results}

In this section, we introduce and analyze an iterative algorithm by hybrid extragradient-like viscosity method for finding a common solution of a systems of generalized equilibrium problems and a generalized mixed equilibrium problem with the constraints of two problems: a finite family of variational inclusions for maximal monotone and inverse strongly monotone mappings and a fixed point problem of infinitely many nonexpansive mappings in a real Hilbert space. Under appropriate conditions imposed on the parameter sequences we will prove strong convergence of the proposed algorithm.

Theorem 21. Let $C$ be a nonempty closed convex subset of a real Hilbert space $H$. Let $N$ be an integer. Let $\Theta, \Theta_{1}, \Theta_{2}$ be three bifunctions from $\mathrm{C} \times \mathrm{C}$ to $\mathbf{R}$ satisfying ( $\mathrm{H1})-(\mathrm{H} 4)$ and $\varphi: C \rightarrow \mathbf{R}$ be a lower semicontinuous and convex functional. Let $R_{i}: C \rightarrow 2^{H}$ be a maximal monotone mapping and let $A, A_{k}: H \rightarrow H$ and $B_{i}: C \rightarrow H$ be $\zeta$ inverse strongly monotone, $\zeta_{k}$-inverse strongly monotone and $\eta_{i}$-inverse strongly monotone, respectively, where $k \in\{1,2\}$ and $i \in\{1,2, \ldots, N\}$. Let $\left\{T_{n}\right\}_{n=1}^{\infty}$ be a sequence of nonexpansive mappings on $H$ and $\left\{\lambda_{n}\right\}$ be a sequence in $(0, b]$ for some $b \in(0,1)$. Let $V$ be a $\bar{\gamma}$-strongly positive bounded linear operator and $f: H \rightarrow H$ be an l-Lipschitzian mapping with $\gamma l<(1+\mu) \bar{\gamma}$. Let $W_{n}$ be the $W$-mapping defined by (9). Assume that $\Omega:=\cap_{n=1}^{\infty} \operatorname{Fix}\left(T_{n}\right) \cap \operatorname{GMEP}(\Theta, \varphi, A) \cap \operatorname{SGEP}(G) \cap$ $\cap_{i=1}^{N} I\left(B_{i}, R_{i}\right) \neq \emptyset$ where $G$ is defined as in Proposition CY. Let $\left\{\alpha_{n}\right\},\left\{\beta_{n}\right\}$ and $\left\{\delta_{n}\right\}$ be three sequences in $[0,1]$. Assume that:

(i) $K: H \rightarrow \mathbf{R}$ is strongly convex with constant $\sigma>0$ and its derivative $K^{\prime}$ is Lipschitz continuous with constant $v>0$ such that the function $x \mapsto\left\langle y-x, K^{\prime}(x)\right\rangle$ is weakly upper semicontinuous for each $y \in H$;

(ii) for each $x \in H$, there exist a bounded subset $D_{x} \subset C$ and $z_{x} \in C$ such that for any $y \notin D_{x}$,

$$
\begin{aligned}
& \Theta\left(y, z_{x}\right)+\varphi\left(z_{x}\right)-\varphi(y) \\
& +\frac{1}{r}\left\langle K^{\prime}(y)-K^{\prime}(x), z_{x}-y\right\rangle<0 ;
\end{aligned}
$$

(iii) $\lim _{n \rightarrow \infty} \alpha_{n}=0, \sum_{n=1}^{\infty} \alpha_{n}=\infty, 0<\liminf _{n \rightarrow \infty} \beta_{n} \leq$ $\limsup _{n \rightarrow \infty} \beta_{n}<1$ and $0<\liminf _{n \rightarrow \infty} \delta_{n} \leq$ $\lim _{\sup _{n \rightarrow \infty}} \delta_{n}<1$; (iv) $\nu_{k} \in\left(0,2 \zeta_{k}\right), k \in\{1,2\}, \mu_{i} \in\left(0,2 \eta_{i}\right), i \in\{1,2, \ldots, N\}$, and $\left\{r_{n}\right\} \subset[0,2 \zeta]$ satisfies

$$
0<\liminf _{n \rightarrow \infty} r_{n} \leq \limsup _{n \rightarrow \infty} r_{n}<2 \zeta
$$

(v) $\lim _{n \rightarrow \infty}\left(\left|\delta_{n+1}-\delta_{n}\right|+\left|r_{n+1}-r_{n}\right|\right)=0$.

Given $x_{1} \in H$ arbitrarily, then the sequence $\left\{x_{n}\right\}$ generated iteratively by

$$
\begin{gathered}
u_{n}=S_{r_{n}}^{(\Theta, \varphi)}\left(I-r_{n} A\right) x_{n}, \\
z_{n}=J_{R_{N}, \mu_{N}}\left(I-\mu_{N} B_{N}\right) J_{R_{N-1}, \mu_{N-1}} \\
\times\left(I-\mu_{N-1} B_{N-1}\right) \cdots J_{R_{1}, \mu_{1}}\left(I-\mu_{1} B_{1}\right) u_{n}, \\
y_{n}=\delta_{n} G z_{n}+\left(1-\delta_{n}\right) W_{n} z_{n}, \\
x_{n+1}=\alpha_{n}\left(u+\gamma f\left(x_{n}\right)\right)+\beta_{n} x_{n} \\
+\left(\left(1-\beta_{n}\right) I-\alpha_{n}(I+\mu V)\right) W_{n} y_{n}, \quad \forall n \geq 1,
\end{gathered}
$$

converges strongly to $x^{*} \in \Omega$ which solves the following optimization problem provided $S_{r}^{(\Theta, \varphi)}$ is firmly nonexpansive:

$$
\min _{x \in \Omega} \frac{\mu}{2}\langle A x, x\rangle+\frac{1}{2}\|x-u\|^{2}-h(x),
$$

where $h$ is the potential function of $\gamma f$.

Proof. Since $\lim _{n \rightarrow \infty} \alpha_{n}=0$ and $0<\liminf _{n \rightarrow \infty} \beta_{n} \leq$ $\limsup \sup _{n \rightarrow \infty} \beta_{n}<1$, we may assume, without loss of generality, that $\alpha_{n} \leq\left(1-\beta_{n}\right)(1+\mu\|V\|)^{-1}$. Since $V$ is a $\bar{\gamma}$ strongly positive bounded linear operator on $H$, we know that

$$
\|V\|=\sup \{\langle V u, u\rangle: u \in H,\|u\|=1\} .
$$

Observe that

$$
\begin{aligned}
\left\langle\left(\left(1-\beta_{n}\right) I-\alpha_{n}(I+\mu V)\right) u, u\right\rangle & =1-\beta_{n}-\alpha_{n}-\alpha_{n} \mu\langle V u, u\rangle \\
& \geq 1-\beta_{n}-\alpha_{n}-\alpha_{n} \mu\|V\| \\
& \geq 0
\end{aligned}
$$

that is, $\left(1-\beta_{n}\right) I-\alpha_{n}(I+\mu V)$ is positive. It follows that

$$
\begin{aligned}
& \left\|\left(1-\beta_{n}\right) I-\alpha_{n}(I+\mu V)\right\| \\
& \quad=\sup \left\{\left\langle\left(\left(1-\beta_{n}\right) I-\alpha_{n}(I+\mu V)\right) u, u\right\rangle: u \in H,\|u\|=1\right\} \\
& \quad=\sup \left\{1-\beta_{n}-\alpha_{n}-\alpha_{n} \mu\langle V u, u\rangle: u \in H,\|u\|=1\right\} \\
& \quad \leq 1-\beta_{n}-\alpha_{n}-\alpha_{n} \mu \bar{\gamma} .
\end{aligned}
$$

Put

$$
\begin{aligned}
\Lambda^{i}= & J_{R_{i}, \mu_{i}}\left(I-\mu_{i} B_{i}\right) J_{R_{i-1}, \mu_{i-1}} \\
& \times\left(I-\mu_{i-1} B_{i-1}\right) \cdots J_{R_{1}, \mu_{1}}\left(I-\mu_{1} B_{1}\right)
\end{aligned}
$$


for all $i \in\{1,2, \ldots, N\}$, and $\Lambda^{0}=I$, where $I$ is the identity mapping on $H$. Then we have that $z_{n}=\Lambda^{N} u_{n}$.

We divide the rest of the proof into several steps.

Step 1. We show that $\left\{x_{n}\right\}$ is bounded. Indeed, take $p \in \Omega$ arbitrarily. Since $p=S_{r_{n}}^{(\Theta, \varphi)}\left(p-r_{n} A p\right), A$ is $\zeta$-inverse strongly monotone and $0 \leq r_{n} \leq 2 \zeta$, we have, for any $n \geq 1$,

$$
\begin{aligned}
\left\|u_{n}-p\right\|^{2}= & \left\|S_{r_{n}}^{(\Theta, \varphi)}\left(I-r_{n} A\right) x_{n}-S_{r_{n}}^{(\Theta, \varphi)}\left(I-r_{n} A\right) p\right\|^{2} \\
\leq & \left\|\left(I-r_{n} A\right) x_{n}-\left(I-r_{n} A\right) p\right\|^{2} \\
= & \left\|\left(x_{n}-p\right)-r_{n}\left(A x_{n}-A p\right)\right\|^{2} \\
= & \left\|x_{n}-p\right\|^{2}-2 r_{n}\left\langle x_{n}-p, A x_{n}-A p\right\rangle \\
& +r_{n}^{2}\left\|A x_{n}-A p\right\|^{2} \\
\leq & \left\|x_{n}-p\right\|^{2}-2 r_{n} \zeta\left\|A x_{n}-A p\right\|^{2}+r_{n}^{2}\left\|A x_{n}-A p\right\|^{2} \\
= & \left\|x_{n}-p\right\|^{2}+r_{n}\left(r_{n}-2 \zeta\right)\left\|A x_{n}-A p\right\|^{2} \\
\leq & \left\|x_{n}-p\right\|^{2} .
\end{aligned}
$$

Since $p=J_{R_{i}, \mu_{i}}\left(I-\mu_{i} B_{i}\right) p, \Lambda^{i} p=p$ and $B_{i}$ is $\eta_{i}$-inverse strongly monotone, where $\mu_{i} \in\left(0,2 \eta_{i}\right), i \in\{1,2, \ldots, N\}$, by Lemma 16 we deduce that for each $n \geq 1$

$$
\begin{aligned}
\| z_{n}- & p \|^{2} \\
= & \| J_{R_{N}, \mu_{N}}\left(I-\mu_{N} B_{N}\right) \Lambda^{N-1} u_{n} \\
& \quad-J_{R_{N}, \mu_{N}}\left(I-\mu_{N} B_{N}\right) \Lambda^{N-1} p \|^{2} \\
\leq & \left\|\left(I-\mu_{N} B_{N}\right) \Lambda^{N-1} u_{n}-\left(I-\mu_{N} B_{N}\right) \Lambda^{N-1} p\right\|^{2} \\
= & \left\|\left(\Lambda^{N-1} u_{n}-\Lambda^{N-1} p\right)-\mu_{N}\left(B_{N} \Lambda^{N-1} u_{n}-B_{N} \Lambda^{N-1} p\right)\right\|^{2} \\
\leq & \left\|\Lambda^{N-1} u_{n}-\Lambda^{N-1} p\right\|^{2} \\
& +\mu_{N}\left(\mu_{N}-2 \eta_{N}\right)\left\|B_{N} \Lambda^{N-1} u_{n}-B_{N} \Lambda^{N-1} p\right\|^{2} \\
\leq & \left\|\Lambda^{N-1} u_{n}-\Lambda^{N-1} p\right\|^{2} \\
\cdots & \\
\leq & \left\|\Lambda^{0} u_{n}-\Lambda^{0} p\right\|^{2}=\left\|u_{n}-p\right\|^{2} .
\end{aligned}
$$

Combining (52) and (53), we have

$$
\left\|z_{n}-p\right\| \leq\left\|x_{n}-p\right\| .
$$

Since $p=G p=T_{v_{1}}^{\Theta_{1}}\left(I-v_{1} A_{1}\right) T_{v_{2}}^{\Theta_{2}}\left(I-v_{2} A_{2}\right) p, A_{k}$ is $\zeta_{k}$ inverse-strongly monotone for $k=1,2$, and $0 \leq \nu_{k} \leq 2 \zeta_{k}$ for $k=1,2$, we deduce that, for any $n \geq 1$,

$$
\begin{aligned}
& \left\|G z_{n}-p\right\|^{2} \\
& =\| T_{v_{1}}^{\Theta_{1}}\left(I-v_{1} A_{1}\right) T_{v_{2}}^{\Theta_{2}}\left(I-v_{2} A_{2}\right) z_{n} \\
& -T_{\nu_{1}}^{\Theta_{1}}\left(I-v_{1} A_{1}\right) T_{v_{2}}^{\Theta_{2}}\left(I-v_{2} A_{2}\right) p \|^{2} \\
& \leq \|\left(I-v_{1} A_{1}\right) T_{v_{2}}^{\Theta_{2}}\left(I-v_{2} A_{2}\right) z_{n} \\
& -\left(I-v_{1} A_{1}\right) T_{v_{2}}^{\Theta_{2}}\left(I-v_{2} A_{2}\right) p \|^{2} \\
& =\|\left[T_{v_{2}}^{\Theta_{2}}\left(I-v_{2} A_{2}\right) z_{n}-T_{v_{2}}^{\Theta_{2}}\left(I-v_{2} A_{2}\right) p\right] \\
& -v_{1}\left[A_{1} T_{\nu_{2}}^{\Theta_{2}}\left(I-v_{2} A_{2}\right) z_{n}-A_{1} T_{v_{2}}^{\Theta_{2}}\left(I-v_{2} A_{2}\right) p\right] \|^{2} \\
& \leq\left\|T_{v_{2}}^{\Theta_{2}}\left(I-v_{2} A_{2}\right) z_{n}-T_{v_{2}}^{\Theta_{2}}\left(I-v_{2} A_{2}\right) p\right\|^{2} \\
& +\nu_{1}\left(\nu_{1}-2 \zeta_{1}\right) \\
& \times\left\|A_{1} T_{v_{2}}^{\Theta_{2}}\left(I-v_{2} A_{2}\right) z_{n}-A_{1} T_{v_{2}}^{\Theta_{2}}\left(I-v_{2} A_{2}\right) p\right\|^{2} \\
& \leq\left\|T_{\nu_{2}}^{\Theta_{2}}\left(I-v_{2} A_{2}\right) z_{n}-T_{\nu_{2}}^{\Theta_{2}}\left(I-v_{2} A_{2}\right) p\right\|^{2} \\
& \leq\left\|\left(I-v_{2} A_{2}\right) z_{n}-\left(I-v_{2} A_{2}\right) p\right\|^{2} \\
& =\left\|\left(z_{n}-p\right)-v_{2}\left(A_{2} z_{n}-A_{2} p\right)\right\|^{2} \\
& \leq\left\|z_{n}-p\right\|^{2}+v_{2}\left(\nu_{2}-2 \zeta_{2}\right)\left\|A_{2} z_{n}-A_{2} p\right\|^{2} \leq\left\|z_{n}-p\right\|^{2} \text {. }
\end{aligned}
$$

(This shows that $G$ is nonexpansive.) Thus, from (54), we get

$$
\begin{aligned}
\left\|y_{n}-p\right\| & =\left\|\delta_{n}\left(G z_{n}-p\right)+\left(1-\delta_{n}\right)\left(W_{n} z_{n}-p\right)\right\| \\
& \leq \delta_{n}\left\|G z_{n}-p\right\|+\left(1-\delta_{n}\right)\left\|W_{n} z_{n}-p\right\| \\
& \leq \delta_{n}\left\|z_{n}-p\right\|+\left(1-\delta_{n}\right)\left\|z_{n}-p\right\| \\
& =\left\|z_{n}-p\right\| \leq\left\|x_{n}-p\right\| .
\end{aligned}
$$

Set $\bar{V}=I+\mu V$. Then from (47) we have

$$
\begin{aligned}
& \left\|x_{n+1}-p\right\| \\
& =\| \alpha_{n}\left(u+\gamma f\left(x_{n}\right)\right)+\beta_{n} x_{n} \\
& \quad+\left(\left(1-\beta_{n}\right) I-\alpha_{n}(I+\mu V)\right) W_{n} y_{n}-p \| \\
& =\| \alpha_{n} u+\alpha_{n}\left(\gamma f\left(x_{n}\right)-\bar{V} p\right)+\beta_{n}\left(x_{n}-p\right) \\
& +\left(\left(1-\beta_{n}\right) I-\alpha_{n} \bar{V}\right)\left(W_{n} y_{n}-p\right) \|
\end{aligned}
$$




$$
\begin{aligned}
& \leq\left\|\left(1-\beta_{n}\right) I-\alpha_{n} \bar{V}\right\|\left\|W_{n} y_{n}-p\right\| \\
& +\beta_{n}\left\|x_{n}-p\right\|+\alpha_{n}\|u\|+\alpha_{n}\left\|\gamma f\left(x_{n}\right)-\bar{V} p\right\| \\
& \leq\left(1-\beta_{n}-\alpha_{n}-\alpha_{n} \mu \bar{\gamma}\right)\left\|y_{n}-p\right\| \\
& +\beta_{n}\left\|x_{n}-p\right\|+\alpha_{n}\|u\|+\alpha_{n}\left\|\gamma f\left(x_{n}\right)-\bar{V} p\right\| \\
& \leq\left(1-\alpha_{n}-\alpha_{n} \mu \bar{\gamma}\right)\left\|x_{n}-p\right\|+\alpha_{n}\|u\| \\
& +\alpha_{n}\left(\gamma\left\|f\left(x_{n}\right)-f(p)\right\|+\|\gamma f(p)-\bar{V} p\|\right) \\
& \leq\left(1-\alpha_{n}-\alpha_{n} \mu \bar{\gamma}\right)\left\|x_{n}-p\right\|+\alpha_{n}\|u\| \\
& +\alpha_{n}\left(\gamma l\left\|x_{n}-p\right\|+\|\gamma f(p)-\bar{V} p\|\right) \\
& \leq\left[1-((1+\mu) \bar{\gamma}-\gamma l) \alpha_{n}\right]\left\|x_{n}-p\right\| \\
& +\alpha_{n}(\|\gamma f(p)-\bar{V} p\|+\|u\|) \\
& =\left[1-((1+\mu) \bar{\gamma}-\gamma l) \alpha_{n}\right]\left\|x_{n}-p\right\|+((1+\mu) \bar{\gamma}-\gamma l) \alpha_{n} \\
& \times \frac{\|\gamma f(p)-\bar{V} p\|+\|u\|}{(1+\mu) \bar{\gamma}-\gamma l} \\
& \leq \max \left\{\left\|x_{n}-p\right\|, \frac{\|\gamma f(p)-\bar{V} p\|+\|u\|}{(1+\mu) \bar{\gamma}-\gamma l}\right\} \text {. }
\end{aligned}
$$

By induction, we get

$$
\left\|x_{n}-p\right\| \leq \max \left\{\left\|x_{0}-p\right\|, \frac{\|\gamma f(p)-\bar{V} p\|+\|u\|}{(1+\mu) \bar{\gamma}-\gamma l}\right\} \text {. }
$$

Therefore, $\left\{x_{n}\right\}$ is bounded and so are the sequences $\left\{u_{n}\right\},\left\{z_{n}\right\},\left\{y_{n}\right\},\left\{f\left(x_{n}\right)\right\}$ and $\left\{W_{n} y_{n}\right\}$.

Step 2. We show that $\left\|x_{n+1}-x_{n}\right\| \rightarrow 0$ as $n \rightarrow \infty$. Indeed, define

$$
x_{n+1}=\beta_{n} x_{n}+\left(1-\beta_{n}\right) w_{n}, \quad \forall n \geq 1 .
$$

Then from the definition of $w_{n}$, we obtain

$$
\begin{aligned}
w_{n+1} & -w_{n} \\
= & \frac{x_{n+2}-\beta_{n+1} x_{n+1}}{1-\beta_{n+1}}-\frac{x_{n+1}-\beta_{n} x_{n}}{1-\beta_{n}} \\
= & \frac{\alpha_{n+1}\left(u+\gamma f\left(x_{n+1}\right)\right)+\left(\left(1-\beta_{n+1}\right) I-\alpha_{n+1} \bar{V}\right) W_{n+1} y_{n+1}}{1-\beta_{n+1}} \\
& -\frac{\alpha_{n}\left(u+\gamma f\left(x_{n}\right)\right)+\left(\left(1-\beta_{n}\right) I-\alpha_{n} \bar{V}\right) W_{n} y_{n}}{1-\beta_{n}}
\end{aligned}
$$

$$
\begin{aligned}
= & \frac{\alpha_{n+1}}{1-\beta_{n+1}}\left(u+\gamma f\left(x_{n+1}\right)\right) \\
& -\frac{\alpha_{n}}{1-\beta_{n}}\left(u+\gamma f\left(x_{n}\right)\right)+W_{n+1} y_{n+1}-W_{n} y_{n} \\
& +\frac{\alpha_{n}}{1-\beta_{n}} \bar{V} W_{n} y_{n}-\frac{\alpha_{n+1}}{1-\beta_{n+1}} \bar{V} W_{n+1} y_{n+1} \\
= & \frac{\alpha_{n+1}}{1-\beta_{n+1}}\left[u+\gamma f\left(x_{n+1}\right)-\bar{V} W_{n+1} y_{n+1}\right] \\
& +\frac{\alpha_{n}}{1-\beta_{n}}\left[\bar{V} W_{n} y_{n}-u-\gamma f\left(x_{n}\right)\right] \\
& +W_{n+1} y_{n+1}-W_{n+1} y_{n}+W_{n+1} y_{n}-W_{n} y_{n} .
\end{aligned}
$$

It follows that

$$
\begin{aligned}
\left\|w_{n+1}-w_{n}\right\|-\left\|x_{n+1}-x_{n}\right\| \\
\leq \frac{\alpha_{n+1}}{1-\beta_{n+1}}\left(\|u\|+\left\|\gamma f\left(x_{n+1}\right)\right\|+\left\|\bar{V} W_{n+1} y_{n+1}\right\|\right) \\
\quad+\frac{\alpha_{n}}{1-\beta_{n}}\left(\left\|\bar{V} W_{n} y_{n}\right\|+\|u\|+\left\|\gamma f\left(x_{n}\right)\right\|\right) \\
\quad+\left\|W_{n+1} y_{n+1}-W_{n+1} y_{n}\right\|+\left\|W_{n+1} y_{n}-W_{n} y_{n}\right\| \\
\quad-\left\|x_{n+1}-x_{n}\right\| \\
\leq \frac{\alpha_{n+1}}{1-\beta_{n+1}}\left(\|u\|+\left\|\gamma f\left(x_{n+1}\right)\right\|+\left\|\bar{V} W_{n+1} y_{n+1}\right\|\right) \\
\quad+\frac{\alpha_{n}}{1-\beta_{n}}\left(\left\|\bar{V} W_{n} y_{n}\right\|+\|u\|+\left\|\gamma f\left(x_{n}\right)\right\|\right) \\
\quad+\left\|W_{n+1} y_{n}-W_{n} y_{n}\right\|+\left\|y_{n+1}-y_{n}\right\|-\left\|x_{n+1}-x_{n}\right\| .
\end{aligned}
$$

From (9), since $W_{n}, T_{n}$ and $U_{n, i}$ are all nonexpansive, we have

$$
\begin{aligned}
\left\|W_{n+1} z_{n}-W_{n} z_{n}\right\| & =\left\|\lambda_{1} T_{1} U_{n+1,2} z_{n}-\lambda_{1} T_{1} U_{n, 2} z_{n}\right\| \\
& \leq \lambda_{1}\left\|U_{n+1,2} z_{n}-U_{n, 2} z_{n}\right\| \\
& =\lambda_{1}\left\|\lambda_{2} T_{2} U_{n+1,3} z_{n}-\lambda_{2} T_{2} U_{n, 3} z_{n}\right\| \\
& \leq \lambda_{1} \lambda_{2}\left\|U_{n+1,3} z_{n}-U_{n, 3} z_{n}\right\| \\
& \ldots \\
& \leq \lambda_{1} \lambda_{2} \cdots \lambda_{n}\left\|U_{n+1, n+1} z_{n}-U_{n, n+1} z_{n}\right\| \\
& \leq M \prod_{i=1}^{n} \lambda_{i}, \\
\left\|W_{n+1} y_{n}-W_{n} y_{n}\right\| & =\left\|\lambda_{1} T_{1} U_{n+1,2} y_{n}-\lambda_{1} T_{1} U_{n, 2} y_{n}\right\| \\
& \leq \lambda_{1}\left\|U_{n+1,2} y_{n}-U_{n, 2} y_{n}\right\| \\
& =\lambda_{1}\left\|\lambda_{2} T_{2} U_{n+1,3} y_{n}-\lambda_{2} T_{2} U_{n, 3} y_{n}\right\| \\
& \leq \lambda_{1} \lambda_{2}\left\|U_{n+1,3} y_{n}-U_{n, 3} y_{n}\right\|
\end{aligned}
$$




$$
\begin{aligned}
& \cdots \\
& \leq \lambda_{1} \lambda_{2} \cdots \lambda_{n}\left\|U_{n+1, n+1} y_{n}-U_{n, n+1} y_{n}\right\| \\
& \leq M \prod_{i=1}^{n} \lambda_{i}
\end{aligned}
$$

where $M$ is a constant such that

$$
\begin{aligned}
& \sup _{n \geq 1}\left\{\left\|U_{n+1, n+1} z_{n}\right\|+\left\|U_{n, n+1} z_{n}\right\|\right\} \leq M, \\
& \sup _{n \geq 1}\left\{\left\|U_{n+1, n+1} y_{n}\right\|+\left\|U_{n, n+1} y_{n}\right\|\right\} \leq M .
\end{aligned}
$$

On the other hand, we estimate $\left\|y_{n+1}-y_{n}\right\|$. Taking into account that $0<\liminf _{n \rightarrow \infty} \beta_{n} \leq \limsup _{n \rightarrow \infty} \beta_{n}<$ $1,0<\liminf _{n \rightarrow \infty} \delta_{n} \leq \limsup _{n \rightarrow \infty} \delta_{n}<1$ and $0<$ $\liminf _{n \rightarrow \infty} r_{n} \leq \limsup _{n \rightarrow \infty} r_{n}<2 \zeta$, we may assume, without loss of generality, that $\left\{r_{n}\right\} \subset[c, d] \subset(0,2 \zeta)$ and $\left\{\beta_{n}\right\},\left\{\delta_{n}\right\} \subset[\widehat{c}, \widehat{d}] \subset(0,1)$. Utilizing Remark 5 and Lemma 16, we have

$$
\begin{aligned}
\left\|z_{n+1}-z_{n}\right\|^{2} & \\
= & \| J_{R_{N}, \mu_{N}}\left(I-\mu_{N} B_{N}\right) \Lambda^{N-1} u_{n+1} \\
& \quad-J_{R_{N}, \mu_{N}}\left(I-\mu_{N} B_{N}\right) \Lambda^{N-1} u_{n} \|^{2} \\
\leq & \left\|\left(I-\mu_{N} B_{N}\right) \Lambda^{N-1} u_{n+1}-\left(I-\mu_{N} B_{N}\right) \Lambda^{N-1} u_{n}\right\|^{2} \\
= & \|\left(\Lambda^{N-1} u_{n+1}-\Lambda^{N-1} u_{n}\right) \\
& \quad-\mu_{N}\left(B_{N} \Lambda^{N-1} u_{n+1}-B_{N} \Lambda^{N-1} u_{n}\right) \|^{2} \\
\leq & \left\|\Lambda^{N-1} u_{n+1}-\Lambda^{N-1} u_{n}\right\|^{2} \\
& +\mu_{N}\left(\mu_{N}-2 \eta_{N}\right)\left\|B_{N} \Lambda^{N-1} u_{n+1}-B_{N} \Lambda^{N-1} u_{n}\right\|^{2} \\
\leq & \left\|\Lambda^{N-1} u_{n+1}-\Lambda^{N-1} u_{n}\right\|^{2} \\
& . \\
\leq & \left\|\Lambda^{0} u_{n+1}-\Lambda^{0} u_{n}\right\|^{2}=\left\|u_{n+1}-u_{n}\right\|^{2}, \\
\|(I- & \left.r_{n+1} A\right) x_{n+1}-\left(I-r_{n} A\right) x_{n} \| \\
= & \left\|x_{n+1}-x_{n}-r_{n+1}\left(A x_{n+1}-A x_{n}\right)+\left(r_{n}-r_{n+1}\right) A x_{n}\right\| \\
\leq & \left\|x_{n+1}-x_{n}-r_{n+1}\left(A x_{n+1}-A x_{n}\right)\right\|+\left|r_{n+1}-r_{n}\right|\left\|A x_{n}\right\| \\
\leq & \left\|x_{n+1}-x_{n}\right\|+\left|r_{n+1}-r_{n}\right|\left\|A x_{n}\right\|,
\end{aligned}
$$

$$
\begin{aligned}
& \left\|u_{n+1}-u_{n}\right\| \\
& =\left\|S_{r_{n+1}}^{(\Theta, \varphi)}\left(I-r_{n+1} A\right) x_{n+1}-S_{r_{n}}^{(\Theta, \varphi)}\left(I-r_{n} A\right) x_{n}\right\| \\
& =\| S_{r_{n+1}}^{(\Theta, \varphi)}\left(I-r_{n+1} A\right) x_{n+1}-S_{r_{n+1}}^{(\Theta, \varphi)}\left(I-r_{n} A\right) x_{n} \\
& +S_{r_{n+1}}^{(\Theta, \varphi)}\left(I-r_{n} A\right) x_{n}-S_{r_{n}}^{(\Theta, \varphi)}\left(I-r_{n} A\right) x_{n} \| \\
& \leq\left\|S_{r_{n+1}}^{(\Theta, \varphi)}\left(I-r_{n+1} A\right) x_{n+1}-S_{r_{n+1}}^{(\Theta, \varphi)}\left(I-r_{n} A\right) x_{n}\right\| \\
& +\left\|S_{r_{n+1}}^{(\Theta, \varphi)}\left(I-r_{n} A\right) x_{n}-S_{r_{n}}^{(\Theta, \varphi)}\left(I-r_{n} A\right) x_{n}\right\| \\
& \leq\left\|\left(I-r_{n+1} A\right) x_{n+1}-\left(I-r_{n} A\right) x_{n}\right\| \\
& +\left\|S_{r_{n+1}}^{(\Theta, \varphi)}\left(I-r_{n} A\right) x_{n}-S_{r_{n}}^{(\Theta, \varphi)}\left(I-r_{n} A\right) x_{n}\right\| \\
& \leq\left\|x_{n+1}-x_{n}\right\|+\left|r_{n+1}-r_{n}\right|\left\|A x_{n}\right\|
\end{aligned}
$$

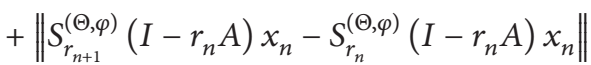

$$
\begin{aligned}
& \leq\left\|x_{n+1}-x_{n}\right\|+\left|r_{n+1}-r_{n}\right|\left\|A x_{n}\right\|+\frac{\left|r_{n+1}-r_{n}\right|}{r_{n+1}} \\
& \cdot \frac{\nu}{\sigma}\left\|S_{r_{n+1}}^{(\Theta, \varphi)}\left(I-r_{n} A\right) x_{n}-\left(I-r_{n} A\right) x_{n}\right\| \\
& \leq\left\|x_{n+1}-x_{n}\right\|+\left|r_{n+1}-r_{n}\right| \\
& \times\left(\left\|A x_{n}\right\|+\frac{\nu}{c \sigma}\left\|S_{r_{n+1}}^{(\Theta, \varphi)}\left(I-r_{n} A\right) x_{n}-\left(I-r_{n} A\right) x_{n}\right\|\right) \\
& \leq\left\|x_{n+1}-x_{n}\right\|+\left|r_{n+1}-r_{n}\right| M_{1} \text {, }
\end{aligned}
$$

where $\sup _{n \geq 1}\left\{\left\|A x_{n}\right\|+(\nu / c \sigma) \| S_{r_{n+1}}^{(\Theta, \varphi)}\left(I-r_{n} A\right) x_{n}-(I-\right.$ $\left.\left.r_{n} A\right) x_{n} \|\right\} \leq M_{1}$ for some $M_{1}>0$.

Note that

$$
\begin{aligned}
y_{n+1} & -y_{n} \\
= & \delta_{n}\left(G z_{n+1}-G z_{n}\right)+\left(\delta_{n+1}-\delta_{n}\right)\left(G z_{n+1}-W_{n+1} z_{n+1}\right) \\
& +\left(1-\delta_{n}\right)\left(W_{n+1} z_{n+1}-W_{n} z_{n}\right) .
\end{aligned}
$$

Since $G$ is nonexpansive, from (62), (64) and (66) it follows that

$$
\begin{aligned}
& \left\|y_{n+1}-y_{n}\right\| \\
& \leq \delta_{n}\left\|G z_{n+1}-G z_{n}\right\|+\left|\delta_{n+1}-\delta_{n}\right|\left\|G z_{n+1}-W_{n+1} z_{n+1}\right\| \\
& \quad+\left(1-\delta_{n}\right)\left\|W_{n+1} z_{n+1}-W_{n} z_{n}\right\| \\
& \leq \delta_{n}\left\|z_{n+1}-z_{n}\right\|+\left|\delta_{n+1}-\delta_{n}\right|\left\|G z_{n+1}-W_{n+1} z_{n+1}\right\| \\
& \quad+\left(1-\delta_{n}\right)\left(\left\|W_{n+1} z_{n+1}-W_{n+1} z_{n}\right\|\right.
\end{aligned}
$$




$$
\begin{aligned}
& \left.+\left\|W_{n+1} z_{n}-W_{n} z_{n}\right\|\right) \\
& \leq \delta_{n}\left\|z_{n+1}-z_{n}\right\|+\left|\delta_{n+1}-\delta_{n}\right|\left\|G z_{n+1}-W_{n+1} z_{n+1}\right\| \\
& +\left(1-\delta_{n}\right)\left(\left\|z_{n+1}-z_{n}\right\|+M \prod_{i=1}^{n} \lambda_{i}\right) \\
& \leq\left\|z_{n+1}-z_{n}\right\|+\left|\delta_{n+1}-\delta_{n}\right|\left\|G z_{n+1}-W_{n+1} z_{n+1}\right\| \\
& \quad+M \prod_{i=1}^{n} \lambda_{i} \\
& \leq\left\|u_{n+1}-u_{n}\right\|+\left|\delta_{n+1}-\delta_{n}\right|\left\|G z_{n+1}-W_{n+1} z_{n+1}\right\| \\
& \quad+M \prod_{i=1}^{n} \lambda_{i} \\
& \leq\left\|x_{n+1}-x_{n}\right\|+\left|r_{n+1}-r_{n}\right| M_{1} \\
& \quad+\left|\delta_{n+1}-\delta_{n}\right|\left\|G z_{n+1}-W_{n+1} z_{n+1}\right\|+M \prod_{i=1}^{n} \lambda_{i} .
\end{aligned}
$$

Utilizing (61), (62)' and (68), we have

$$
\begin{aligned}
&\left\|w_{n+1}-w_{n}\right\|-\left\|x_{n+1}-x_{n}\right\| \\
& \leq \frac{\alpha_{n+1}}{1-\beta_{n+1}}\left(\|u\|+\left\|\gamma f\left(x_{n+1}\right)\right\|+\left\|\bar{V} W_{n+1} y_{n+1}\right\|\right) \\
&+\frac{\alpha_{n}}{1-\beta_{n}}\left(\left\|\bar{V} W_{n} y_{n}\right\|+\|u\|+\left\|\gamma f\left(x_{n}\right)\right\|\right) \\
&+\left\|W_{n+1} y_{n}-W_{n} y_{n}\right\|+\left\|y_{n+1}-y_{n}\right\|-\left\|x_{n+1}-x_{n}\right\| \\
& \leq \frac{\alpha_{n+1}}{1-\beta_{n+1}}\left(\|u\|+\left\|\gamma f\left(x_{n+1}\right)\right\|+\left\|\bar{V} W_{n+1} y_{n+1}\right\|\right) \\
&+\frac{\alpha_{n}}{1-\beta_{n}}\left(\left\|\bar{V} W_{n} y_{n}\right\|+\|u\|+\left\|\gamma f\left(x_{n}\right)\right\|\right) \\
&+M \prod_{i=1}^{n} \lambda_{i}+\left\|x_{n+1}-x_{n}\right\|+\left|r_{n+1}-r_{n}\right| M_{1} \\
&+\left|\delta_{n+1}-\delta_{n}\right|\left\|G z_{n+1}-W_{n+1} z_{n+1}\right\| \\
&+M \prod_{i=1}^{n} \lambda_{i}-\left\|x_{n+1}-x_{n}\right\| \\
& \leq \frac{\alpha_{n+1}}{1-\widehat{d}}\left(\|u\|+\left\|\gamma f\left(x_{n+1}\right)\right\|+\left\|\bar{V} W_{n+1} y_{n+1}\right\|\right) \\
&+\frac{\alpha_{n}}{1-\widehat{d}}\left(\left\|\bar{V} W_{n} y_{n}\right\|+\|u\|+\left\|\gamma f\left(x_{n}\right)\right\|\right) \\
&+2 M \prod_{i=1}^{n} \lambda_{i}+\left|r_{n+1}-r_{n}\right| M_{1} \\
&+\left|\delta_{n+1}-\delta_{n}\right|\left\|G z_{n+1}-W_{n+1} z_{n+1}\right\|
\end{aligned}
$$

$$
\begin{aligned}
& \leq M_{2}\left(\alpha_{n+1}+\alpha_{n}+\prod_{i=1}^{n} \lambda_{i}\right. \\
& \left.\quad+\left|r_{n+1}-r_{n}\right|+\left|\delta_{n+1}-\delta_{n}\right|\right) \\
& \leq M_{2}\left(\alpha_{n+1}+\alpha_{n}+b^{n}+\left|r_{n+1}-r_{n}\right|+\left|\delta_{n+1}-\delta_{n}\right|\right),
\end{aligned}
$$

where $\sup _{n \geq 1}\left\{(1 /(1-\widehat{d}))\left(\|u\|+\left\|\gamma f\left(x_{n}\right)\right\|+\left\|\bar{V} W_{n} y_{n}\right\|\right)+\| G z_{n}-\right.$ $\left.W_{n} z_{n} \|+M_{1}+2 M\right\} \leq M_{2}$ for some $M_{2}>0$. Since $b \in$ $(0,1), \lim _{n \rightarrow \infty} \alpha_{n}=0$ and $\lim _{n \rightarrow \infty}\left(\left|\delta_{n+1}-\delta_{n}\right|+\left|r_{n+1}-r_{n}\right|\right)=0$, we deduce from (69) that

$$
\limsup _{n \rightarrow \infty}\left(\left\|w_{n+1}-w_{n}\right\|-\left\|x_{n+1}-x_{n}\right\|\right)=0 .
$$

Since $x_{n+1}=\beta_{n} x_{n}+\left(1-\beta_{n}\right) w_{n}$ for all $n \geq 1$, by Lemma 14 we obtain from $0<\liminf \inf _{n \rightarrow \infty} \beta_{n} \leq \lim \sup _{n \rightarrow \infty} \beta_{n}<1$ that

$$
\lim _{n \rightarrow \infty}\left\|w_{n}-x_{n}\right\|=0,
$$

which immediately yields

$$
\lim _{n \rightarrow \infty}\left\|x_{n+1}-x_{n}\right\|=\lim _{n \rightarrow \infty}\left(1-\beta_{n}\right)\left\|w_{n}-x_{n}\right\|=0 .
$$

Step 3. $\left\|y_{n}-G z_{n}\right\| \rightarrow 0$ as $n \rightarrow \infty$.

Indeed, utilizing Lemmas 6 and 7(b) we obtain from (47) and (54) that

$$
\begin{aligned}
\left\|x_{n+1}-p\right\|^{2} & \| \alpha_{n}\left(\left(u+\gamma f\left(x_{n}\right)\right)-\bar{V} W_{n} y_{n}\right) \\
& +\beta_{n}\left(x_{n}-p\right)+\left(1-\beta_{n}\right)\left(W_{n} y_{n}-p\right) \|^{2} \\
\leq & \left\|\beta_{n}\left(x_{n}-p\right)+\left(1-\beta_{n}\right)\left(W_{n} y_{n}-p\right)\right\|^{2} \\
& +2 \alpha_{n}\left\langle\left(u+\gamma f\left(x_{n}\right)\right)-\bar{V} W_{n} y_{n}, x_{n+1}-p\right\rangle \\
= & \beta_{n}\left\|x_{n}-p\right\|^{2}+\left(1-\beta_{n}\right)\left\|W_{n} y_{n}-p\right\|^{2} \\
& -\beta_{n}\left(1-\beta_{n}\right)\left\|x_{n}-W_{n} y_{n}\right\|^{2} \\
& +2 \alpha_{n}\left\|\left(u+\gamma f\left(x_{n}\right)\right)-\bar{V} W_{n} y_{n}\right\|\left\|x_{n+1}-p\right\| \\
\leq & \beta_{n}\left\|x_{n}-p\right\|^{2}+\left(1-\beta_{n}\right)\left\|y_{n}-p\right\|^{2} \\
& -\beta_{n}\left(1-\beta_{n}\right)\left\|x_{n}-W_{n} y_{n}\right\|^{2} \\
& +2 \alpha_{n}\left\|\left(u+\gamma f\left(x_{n}\right)\right)-\bar{V} W_{n} y_{n}\right\|\left\|x_{n+1}-p\right\| \\
\leq & \beta_{n}\left\|x_{n}-p\right\|^{2}+\left(1-\beta_{n}\right)\left\|x_{n}-p\right\|^{2} \\
& -\beta_{n}\left(1-\beta_{n}\right)\left\|x_{n}-W_{n} y_{n}\right\|^{2} \\
& +2 \alpha_{n}\left\|\left(u+\gamma f\left(x_{n}\right)\right)-\bar{V} W_{n} y_{n}\right\|\left\|x_{n+1}-p\right\| \\
= & \left\|x_{n}-p\right\|^{2}-\beta_{n}\left(1-\beta_{n}\right)\left\|x_{n}-W_{n} y_{n}\right\|^{2} \\
& +2 \alpha_{n}\left\|\left(u+\gamma f\left(x_{n}\right)\right)-\bar{V} W_{n} y_{n}\right\|\left\|x_{n+1}-p\right\|,
\end{aligned}
$$


which leads to

$$
\begin{aligned}
\widehat{c}(1-\widehat{d}) & \left\|x_{n}-W_{n} y_{n}\right\|^{2} \\
\leq & \beta_{n}\left(1-\beta_{n}\right)\left\|x_{n}-W_{n} y_{n}\right\|^{2} \\
\leq & \left\|x_{n}-p\right\|^{2}-\left\|x_{n+1}-p\right\|^{2} \\
& +2 \alpha_{n}\left\|\left(u+\gamma f\left(x_{n}\right)\right)-\bar{V} W_{n} y_{n}\right\|\left\|x_{n+1}-p\right\| \\
\leq & \left\|x_{n}-x_{n+1}\right\|\left(\left\|x_{n}-p\right\|+\left\|x_{n+1}-p\right\|\right) \\
& +2 \alpha_{n}\left\|\left(u+\gamma f\left(x_{n}\right)\right)-\bar{V} W_{n} y_{n}\right\|\left\|x_{n+1}-p\right\| .
\end{aligned}
$$

Since $\lim _{n \rightarrow \infty}\left\|x_{n}-x_{n+1}\right\|=0$ and $\lim _{n \rightarrow \infty} \alpha_{n}=0$, we deduce from the boundedness of $\left\{x_{n}\right\},\left\{y_{n}\right\},\left\{f\left(x_{n}\right)\right\}$ and $\left\{W_{n} y_{n}\right\}$ that

$$
\lim _{n \rightarrow \infty}\left\|x_{n}-W_{n} y_{n}\right\|=0
$$

Also, by Lemma 7(b) we deduce from (47) and (54) that

$$
\begin{aligned}
\left\|y_{n}-p\right\|^{2}= & \delta_{n}\left\|G z_{n}-p\right\|^{2}+\left(1-\delta_{n}\right)\left\|W_{n} z_{n}-p\right\|^{2} \\
& -\delta_{n}\left(1-\delta_{n}\right)\left\|G z_{n}-W_{n} z_{n}\right\|^{2} \\
\leq & \delta_{n}\left\|z_{n}-p\right\|^{2}+\left(1-\delta_{n}\right)\left\|z_{n}-p\right\|^{2} \\
& -\delta_{n}\left(1-\delta_{n}\right)\left\|G z_{n}-W_{n} z_{n}\right\|^{2} \\
= & \left\|z_{n}-p\right\|^{2}-\delta_{n}\left(1-\delta_{n}\right)\left\|G z_{n}-W_{n} z_{n}\right\|^{2} \\
\leq & \left\|x_{n}-p\right\|^{2}-\delta_{n}\left(1-\delta_{n}\right)\left\|G z_{n}-W_{n} z_{n}\right\|^{2} .
\end{aligned}
$$

From (73) and (76) we get

$$
\begin{aligned}
&\left\|x_{n+1}-p\right\|^{2} \\
& \leq \beta_{n}\left\|x_{n}-p\right\|^{2}+\left(1-\beta_{n}\right)\left\|y_{n}-p\right\|^{2} \\
&-\beta_{n}\left(1-\beta_{n}\right)\left\|x_{n}-W_{n} y_{n}\right\|^{2} \\
&+2 \alpha_{n}\left\|\left(u+\gamma f\left(x_{n}\right)\right)-\bar{V} W_{n} y_{n}\right\|\left\|x_{n+1}-p\right\| \\
& \leq \beta_{n}\left\|x_{n}-p\right\|^{2}+\left(1-\beta_{n}\right)\left\|y_{n}-p\right\|^{2} \\
&+2 \alpha_{n}\left\|\left(u+\gamma f\left(x_{n}\right)\right)-\bar{V} W_{n} y_{n}\right\|\left\|x_{n+1}-p\right\| \\
& \leq \beta_{n}\left\|x_{n}-p\right\|^{2}+\left(1-\beta_{n}\right) \\
& \times\left[\left\|x_{n}-p\right\|^{2}-\delta_{n}\left(1-\delta_{n}\right)\left\|G z_{n}-W_{n} z_{n}\right\|^{2}\right] \\
&+2 \alpha_{n}\left\|\left(u+\gamma f\left(x_{n}\right)\right)-\bar{V} W_{n} y_{n}\right\|\left\|x_{n+1}-p\right\| \\
&=\left\|x_{n}-p\right\|^{2}-\left(1-\beta_{n}\right) \delta_{n}\left(1-\delta_{n}\right)\left\|G z_{n}-W_{n} z_{n}\right\|^{2} \\
&+2 \alpha_{n}\left\|\left(u+\gamma f\left(x_{n}\right)\right)-\bar{V} W_{n} y_{n}\right\|\left\|x_{n+1}-p\right\|,
\end{aligned}
$$

Since $\lim _{n \rightarrow \infty} \alpha_{n}=0$ and $\lim _{n \rightarrow \infty}\left\|x_{n+1}-x_{n}\right\|=0$, we deduce from the boundedness of $\left\{x_{n}\right\},\left\{y_{n}\right\},\left\{f\left(x_{n}\right)\right\}$ and $\left\{W_{n} y_{n}\right\}$ that

$$
\lim _{n \rightarrow \infty}\left\|G z_{n}-W_{n} z_{n}\right\|=0 .
$$

So, it follows that

$$
\lim _{n \rightarrow \infty}\left\|y_{n}-G z_{n}\right\|=\lim _{n \rightarrow \infty}\left(1-\delta_{n}\right)\left\|W_{n} z_{n}-G z_{n}\right\|=0 .
$$

Step 4. $\left\|x_{n}-u_{n}\right\| \rightarrow 0,\left\|u_{n}-z_{n}\right\| \rightarrow 0,\left\|z_{n}-G z_{n}\right\| \rightarrow 0$ and $\left\|z_{n}-W z_{n}\right\| \rightarrow 0$ as $n \rightarrow \infty$.

Indeed, for $p \in \Omega$, we find that

$$
\begin{aligned}
\| u_{n} & -p \|^{2} \\
& =\left\|S_{r_{n}}^{(\Theta, \varphi)}\left(I-r_{n} A\right) x_{n}-S_{r_{n}}^{(\Theta, \varphi)}\left(I-r_{n} A\right) p\right\|^{2} \\
& \leq\left\|\left(I-r_{n} A\right) x_{n}-\left(I-r_{n} A\right) p\right\|^{2} \\
& =\left\|x_{n}-p-r_{n}\left(A x_{n}-A p\right)\right\|^{2} \\
& \leq\left\|x_{n}-p\right\|^{2}+r_{n}\left(r_{n}-2 \zeta\right)\left\|A x_{n}-A p\right\|^{2} .
\end{aligned}
$$

From (47), (53) and (81), we obtain

$$
\begin{aligned}
\left\|y_{n}-p\right\|^{2} & \leq \delta_{n}\left\|G z_{n}-p\right\|^{2}+\left(1-\delta_{n}\right)\left\|W_{n} z_{n}-p\right\|^{2} \\
& \leq\left\|z_{n}-p\right\|^{2} \leq\left\|u_{n}-p\right\|^{2} \\
& \leq\left\|x_{n}-p\right\|^{2}+r_{n}\left(r_{n}-2 \zeta\right)\left\|A x_{n}-A p\right\|^{2},
\end{aligned}
$$


which together with (73), implies that

$$
\begin{aligned}
\| x_{n+1} & -p \|^{2} \\
\leq & \beta_{n}\left\|x_{n}-p\right\|^{2}+\left(1-\beta_{n}\right)\left\|y_{n}-p\right\|^{2} \\
& -\beta_{n}\left(1-\beta_{n}\right)\left\|x_{n}-W_{n} y_{n}\right\|^{2} \\
& +2 \alpha_{n}\left\|\left(u+\gamma f\left(x_{n}\right)\right)-\bar{V} W_{n} y_{n}\right\|\left\|x_{n+1}-p\right\| \\
\leq & \beta_{n}\left\|x_{n}-p\right\|^{2}+\left(1-\beta_{n}\right)\left\|y_{n}-p\right\|^{2} \\
& +2 \alpha_{n}\left\|\left(u+\gamma f\left(x_{n}\right)\right)-\bar{V} W_{n} y_{n}\right\|\left\|x_{n+1}-p\right\| \\
\leq & \beta_{n}\left\|x_{n}-p\right\|^{2}+\left(1-\beta_{n}\right) \\
& \times\left[\left\|x_{n}-p\right\|^{2}+r_{n}\left(r_{n}-2 \zeta\right)\left\|A x_{n}-A p\right\|^{2}\right] \\
& +2 \alpha_{n}\left\|\left(u+\gamma f\left(x_{n}\right)\right)-\bar{V} W_{n} y_{n}\right\|\left\|x_{n+1}-p\right\| \\
= & \left\|x_{n}-p\right\|^{2}+\left(1-\beta_{n}\right) r_{n}\left(r_{n}-2 \zeta\right)\left\|A x_{n}-A p\right\|^{2} \\
& +2 \alpha_{n}\left\|\left(u+\gamma f\left(x_{n}\right)\right)-\bar{V} W_{n} y_{n}\right\|\left\|x_{n+1}-p\right\| .
\end{aligned}
$$

So, it follows that

$$
\begin{aligned}
(1-\widehat{d}) & c(2 \zeta-d)\left\|A x_{n}-A p\right\|^{2} \\
\leq & \left(1-\beta_{n}\right) r_{n}\left(2 \zeta-r_{n}\right)\left\|A x_{n}-A p\right\|^{2} \\
\leq & \left\|x_{n}-p\right\|^{2}-\left\|x_{n+1}-p\right\|^{2} \\
& +2 \alpha_{n}\left\|\left(u+\gamma f\left(x_{n}\right)\right)-\bar{V} W_{n} y_{n}\right\|\left\|x_{n+1}-p\right\| \\
\leq & \left\|x_{n}-x_{n+1}\right\|\left(\left\|x_{n}-p\right\|+\left\|x_{n+1}-p\right\|\right) \\
& +2 \alpha_{n}\left\|\left(u+\gamma f\left(x_{n}\right)\right)-\bar{V} W_{n} y_{n}\right\|\left\|x_{n+1}-p\right\| .
\end{aligned}
$$

Since $\lim _{n \rightarrow \infty} \alpha_{n}=0$ and $\lim _{n \rightarrow \infty}\left\|x_{n}-x_{n+1}\right\|=0$, from the boundedness of $\left\{x_{n}\right\},\left\{y_{n}\right\},\left\{W_{n} y_{n}\right\}$ and $\left\{f\left(x_{n}\right)\right\}$ we get

$$
\lim _{n \rightarrow \infty}\left\|A x_{n}-A p\right\|=0
$$

Furthermore, from the firm nonexpansivity of $S_{r_{n}}^{(\Theta, \varphi)}$, we have

$$
\begin{aligned}
\| u_{n}- & p \|^{2} \\
& =\left\|S_{r_{n}}^{(\Theta, \varphi)}\left(I-r_{n} A\right) x_{n}-S_{r_{n}}^{(\Theta, \varphi)}\left(I-r_{n} A\right) p\right\|^{2}
\end{aligned}
$$

$$
\begin{aligned}
& \leq\left\langle\left(I-r_{n} A\right) x_{n}-\left(I-r_{n} A\right) p, u_{n}-p\right\rangle \\
& =\frac{1}{2}\left[\left\|\left(I-r_{n} A\right) x_{n}-\left(I-r_{n} A\right) p\right\|^{2}+\left\|u_{n}-p\right\|^{2}\right. \\
& \left.-\left\|\left(I-r_{n} A\right) x_{n}-\left(I-r_{n} A\right) p-\left(u_{n}-p\right)\right\|^{2}\right] \\
& \leq \frac{1}{2}\left[\left\|x_{n}-p\right\|^{2}+\left\|u_{n}-p\right\|^{2}\right. \\
& \left.-\left\|x_{n}-u_{n}-r_{n}\left(A x_{n}-A p\right)\right\|^{2}\right] \\
& =\frac{1}{2}\left[\left\|x_{n}-p\right\|^{2}+\left\|u_{n}-p\right\|^{2}-\left\|x_{n}-u_{n}\right\|^{2}\right. \\
& \left.+2 r_{n}\left\langle A x_{n}-A p, x_{n}-u_{n}\right\rangle-r_{n}^{2}\left\|A x_{n}-A p\right\|^{2}\right],
\end{aligned}
$$

which implies that

$$
\begin{aligned}
\left\|u_{n}-p\right\|^{2} \leq & \left\|x_{n}-p\right\|^{2}-\left\|x_{n}-u_{n}\right\|^{2} \\
& +2 r_{n}\left\|A x_{n}-A p\right\|\left\|x_{n}-u_{n}\right\| .
\end{aligned}
$$

From (47) and (87), we have

$$
\begin{aligned}
\left\|y_{n}-p\right\|^{2} \leq & \delta_{n}\left\|G z_{n}-p\right\|^{2}+\left(1-\delta_{n}\right)\left\|W_{n} z_{n}-p\right\|^{2} \\
\leq & \left\|z_{n}-p\right\|^{2} \leq\left\|u_{n}-p\right\|^{2} \\
\leq & \left\|x_{n}-p\right\|^{2}-\left\|x_{n}-u_{n}\right\|^{2} \\
& +2 r_{n}\left\|A x_{n}-A p\right\|\left\|x_{n}-u_{n}\right\|
\end{aligned}
$$

which together with (73), implies that

$$
\begin{aligned}
\| x_{n+1} & -p \|^{2} \\
\leq & \beta_{n}\left\|x_{n}-p\right\|^{2}+\left(1-\beta_{n}\right)\left\|y_{n}-p\right\|^{2} \\
& +2 \alpha_{n}\left\|\left(u+\gamma f\left(x_{n}\right)\right)-\bar{V} W_{n} y_{n}\right\|\left\|x_{n+1}-p\right\| \\
\leq & \beta_{n}\left\|x_{n}-p\right\|^{2}+\left(1-\beta_{n}\right) \\
& \times\left[\left\|x_{n}-p\right\|^{2}-\left\|x_{n}-u_{n}\right\|^{2}+2 r_{n}\left\|A x_{n}-A p\right\|\left\|x_{n}-u_{n}\right\|\right] \\
& +2 \alpha_{n}\left\|\left(u+\gamma f\left(x_{n}\right)\right)-\bar{V} W_{n} y_{n}\right\|\left\|x_{n+1}-p\right\| \\
\leq & \left\|x_{n}-p\right\|^{2}-\left(1-\beta_{n}\right)\left\|x_{n}-u_{n}\right\|^{2} \\
& +2 r_{n}\left\|A x_{n}-A p\right\|\left\|x_{n}-u_{n}\right\| \\
& +2 \alpha_{n}\left\|\left(u+\gamma f\left(x_{n}\right)\right)-\bar{V} W_{n} y_{n}\right\|\left\|x_{n+1}-p\right\| .
\end{aligned}
$$

So, it follows that

$$
\begin{aligned}
& (1-\hat{d})\left\|x_{n}-u_{n}\right\|^{2} \\
& \quad \leq\left(1-\beta_{n}\right)\left\|x_{n}-u_{n}\right\|^{2}
\end{aligned}
$$




$$
\begin{aligned}
\leq & \left\|x_{n}-p\right\|^{2}-\left\|x_{n+1}-p\right\|^{2} \\
& +2 r_{n}\left\|A x_{n}-A p\right\|\left\|x_{n}-u_{n}\right\| \\
& +2 \alpha_{n}\left\|\left(u+\gamma f\left(x_{n}\right)\right)-\bar{V} W_{n} y_{n}\right\|\left\|x_{n+1}-p\right\| \\
\leq & \left\|x_{n}-x_{n+1}\right\|\left(\left\|x_{n}-p\right\|+\left\|x_{n+1}-p\right\|\right) \\
& +2 r_{n}\left\|A x_{n}-A p\right\|\left\|x_{n}-u_{n}\right\| \\
& +2 \alpha_{n}\left\|\left(u+\gamma f\left(x_{n}\right)\right)-\bar{V} W_{n} y_{n}\right\|\left\|x_{n+1}-p\right\| .
\end{aligned}
$$

Since $\lim _{n \rightarrow \infty} \alpha_{n}=0$ and $\lim _{n \rightarrow \infty}\left\|x_{n}-x_{n+1}\right\|=0$, from (85) and the boundedness of $\left\{x_{n}\right\},\left\{y_{n}\right\},\left\{W_{n} y_{n}\right\}$ and $\left\{f\left(x_{n}\right)\right\}$ we get

$$
\lim _{n \rightarrow \infty}\left\|x_{n}-u_{n}\right\|=0 \text {. }
$$

Next we show that $\lim _{n \rightarrow \infty}\left\|A_{i} \Lambda^{i} u_{n}-A_{i} p\right\|=0, i=$ $1,2, \ldots, N$. Observe that

$$
\begin{aligned}
\| \Lambda^{i} u_{n} & -p \|^{2} \\
& =\left\|J_{R_{i}, \mu_{i}}\left(I-\mu_{i} B_{i}\right) \Lambda^{i-1} u_{n}-J_{R_{i}, \mu_{i}}\left(I-\mu_{i} B_{i}\right) p\right\|^{2} \\
& \leq\left\|\left(I-\mu_{i} B_{i}\right) \Lambda^{i-1} u_{n}-\left(I-\mu_{i} B_{i}\right) p\right\|^{2} \\
& \leq\left\|\Lambda^{i-1} u_{n}-p\right\|^{2}+\mu_{i}\left(\mu_{i}-2 \eta_{i}\right)\left\|B_{i} \Lambda^{i-1} u_{n}-B_{i} p\right\|^{2} \\
& \leq\left\|u_{n}-p\right\|^{2}+\mu_{i}\left(\mu_{i}-2 \eta_{i}\right)\left\|B_{i} \Lambda^{i-1} u_{n}-B_{i} p\right\|^{2} \\
& \leq\left\|x_{n}-p\right\|^{2}+\mu_{i}\left(\mu_{i}-2 \eta_{i}\right)\left\|B_{i} \Lambda^{i-1} u_{n}-B_{i} p\right\|^{2} .
\end{aligned}
$$

From (47) and (92), we have

$$
\begin{aligned}
\left\|y_{n}-p\right\|^{2} & \leq \delta_{n}\left\|G z_{n}-p\right\|^{2}+\left(1-\delta_{n}\right)\left\|W_{n} z_{n}-p\right\|^{2} \\
& \leq\left\|z_{n}-p\right\|^{2} \leq\left\|\Lambda^{i} u_{n}-p\right\|^{2} \\
& \leq\left\|x_{n}-p\right\|^{2}+\mu_{i}\left(\mu_{i}-2 \eta_{i}\right)\left\|B_{i} \Lambda^{i-1} u_{n}-B_{i} p\right\|^{2},
\end{aligned}
$$

which together with (73), implies that

$$
\begin{aligned}
&\left\|x_{n+1}-p\right\|^{2} \\
& \leq \beta_{n}\left\|x_{n}-p\right\|^{2}+\left(1-\beta_{n}\right)\left\|y_{n}-p\right\|^{2} \\
&+2 \alpha_{n}\left\|\left(u+\gamma f\left(x_{n}\right)\right)-\bar{V} W_{n} y_{n}\right\|\left\|x_{n+1}-p\right\| \\
& \leq \beta_{n}\left\|x_{n}-p\right\|^{2}+\left(1-\beta_{n}\right) \\
& \times\left[\left\|x_{n}-p\right\|^{2}+\mu_{i}\left(\mu_{i}-2 \eta_{i}\right)\left\|B_{i} \Lambda^{i-1} u_{n}-B_{i} p\right\|^{2}\right] \\
&+2 \alpha_{n}\left\|\left(u+\gamma f\left(x_{n}\right)\right)-\bar{V} W_{n} y_{n}\right\|\left\|x_{n+1}-p\right\| \\
&=\left\|x_{n}-p\right\|^{2}+\left(1-\beta_{n}\right) \mu_{i}\left(\mu_{i}-2 \eta_{i}\right)\left\|B_{i} \Lambda^{i-1} u_{n}-B_{i} p\right\|^{2} \\
&+2 \alpha_{n}\left\|\left(u+\gamma f\left(x_{n}\right)\right)-\bar{V} W_{n} y_{n}\right\|\left\|x_{n+1}-p\right\| .
\end{aligned}
$$

So, it follows that

$$
\begin{aligned}
(1-\widehat{d}) & \mu_{i}\left(2 \eta_{i}-\mu_{i}\right)\left\|B_{i} \Lambda^{i-1} u_{n}-B_{i} p\right\|^{2} \\
\leq & \left(1-\beta_{n}\right) \mu_{i}\left(2 \eta_{i}-\mu_{i}\right)\left\|B_{i} \Lambda^{i-1} u_{n}-B_{i} p\right\|^{2} \\
\leq & \left\|x_{n}-p\right\|^{2}-\left\|x_{n+1}-p\right\|^{2} \\
& +2 \alpha_{n}\left\|\left(u+\gamma f\left(x_{n}\right)\right)-\bar{V} W_{n} y_{n}\right\|\left\|x_{n+1}-p\right\| \\
\leq & \left\|x_{n}-x_{n+1}\right\|\left(\left\|x_{n}-p\right\|+\left\|x_{n+1}-p\right\|\right) \\
& +2 \alpha_{n}\left\|\left(u+\gamma f\left(x_{n}\right)\right)-\bar{V} W_{n} y_{n}\right\|\left\|x_{n+1}-p\right\| .
\end{aligned}
$$

Since $\mu_{i} \in\left(0,2 \eta_{i}\right), \quad i=1,2, \ldots, N, \lim _{n \rightarrow \infty} \alpha_{n}=0$ and $\lim _{n \rightarrow \infty}\left\|x_{n}-x_{n+1}\right\|=0$, from the boundedness of $\left\{x_{n}\right\},\left\{y_{n}\right\},\left\{W_{n} y_{n}\right\}$ and $\left\{f\left(x_{n}\right)\right\}$ we get

$$
\lim _{n \rightarrow \infty}\left\|B_{i} \Lambda^{i-1} u_{n}-B_{i} p\right\|=0, \quad \forall i \in\{1,2, \ldots, N\}
$$

By Lemmas 7 (a) and 16, we obtain

$$
\begin{aligned}
\left\|\Lambda^{i} u_{n}-p\right\|^{2} & \\
= & \left\|J_{R_{i}, \mu_{i}}\left(I-\mu_{i} B_{i}\right) \Lambda^{i-1} u_{n}-J_{R_{i}, \mu_{i}}\left(I-\mu_{i} B_{i}\right) p\right\|^{2} \\
\leq & \left\langle\left(I-\mu_{i} B_{i}\right) \Lambda^{i-1} u_{n}-\left(I-\mu_{i} B_{i}\right) p, \Lambda^{i} u_{n}-p\right\rangle \\
= & \frac{1}{2}\left(\left\|\left(I-\mu_{i} B_{i}\right) \Lambda^{i-1} u_{n}-\left(I-\mu_{i} B_{i}\right) p\right\|^{2}+\left\|\Lambda^{i} u_{n}-p\right\|^{2}\right. \\
& \left.\quad-\left\|\left(I-\mu_{i} B_{i}\right) \Lambda^{i-1} u_{n}-\left(I-\mu_{i} B_{i}\right) p-\left(\Lambda^{i} u_{n}-p\right)\right\|^{2}\right) \\
& \frac{1}{2}\left(\left\|\Lambda^{i-1} u_{n}-p\right\|^{2}+\left\|\Lambda^{i} u_{n}-p\right\|^{2}\right. \\
& \left.\quad-\left\|\Lambda^{i-1} u_{n}-\Lambda^{i} u_{n}-\mu_{i}\left(B_{i} \Lambda^{i-1} u_{n}-B_{i} p\right)\right\|^{2}\right) \\
\leq & \frac{1}{2}\left(\left\|u_{n}-p\right\|^{2}+\left\|\Lambda^{i} u_{n}-p\right\|^{2}\right. \\
& \left.\quad-\left\|\Lambda^{i-1} u_{n}-\Lambda^{i} u_{n}-\mu_{i}\left(B_{i} \Lambda^{i-1} u_{n}-B_{i} p\right)\right\|^{2}\right) \\
\leq & \frac{1}{2}\left(\left\|x_{n}-p\right\|^{2}+\left\|\Lambda^{i} u_{n}-p\right\|_{n}^{2}\right. \\
&
\end{aligned}
$$


which implies that

$$
\begin{aligned}
\left\|\Lambda^{i} u_{n}-p\right\|^{2} \leq & \left\|x_{n}-p\right\|^{2} \\
& -\left\|\Lambda^{i-1} u_{n}-\Lambda^{i} u_{n}-\mu_{i}\left(B_{i} \Lambda^{i-1} u_{n}-B_{i} p\right)\right\|^{2} \\
= & \left\|x_{n}-p\right\|^{2}-\left\|\Lambda^{i-1} u_{n}-\Lambda^{i} u_{n}\right\|^{2} \\
& -\mu_{i}^{2}\left\|B_{i} \Lambda^{i-1} u_{n}-B_{i} p\right\|^{2} \\
& +2 \mu_{i}\left\langle\Lambda^{i-1} u_{n}-\Lambda^{i} u_{n}, B_{i} \Lambda^{i-1} u_{n}-B_{i} p\right\rangle \\
\leq & \left\|x_{n}-p\right\|^{2}-\left\|\Lambda^{i-1} u_{n}-\Lambda^{i} u_{n}\right\|^{2} \\
& +2 \mu_{i}\left\|\Lambda^{i-1} u_{n}-\Lambda^{i} u_{n}\right\|\left\|B_{i} \Lambda^{i-1} u_{n}-B_{i} p\right\|
\end{aligned}
$$

From (47) and (98), we have

$$
\begin{aligned}
\left\|y_{n}-p\right\|^{2} \leq & \delta_{n}\left\|G z_{n}-p\right\|^{2}+\left(1-\delta_{n}\right)\left\|W_{n} z_{n}-p\right\|^{2} \\
\leq & \left\|z_{n}-p\right\|^{2} \leq\left\|\Lambda^{i} u_{n}-p\right\|^{2} \\
\leq & \left\|x_{n}-p\right\|^{2}-\left\|\Lambda^{i-1} u_{n}-\Lambda^{i} u_{n}\right\|^{2} \\
& +2 \mu_{i}\left\|\Lambda^{i-1} u_{n}-\Lambda^{i} u_{n}\right\|\left\|B_{i} \Lambda^{i-1} u_{n}-B_{i} p\right\|,
\end{aligned}
$$

which together with (73), implies that

$$
\begin{aligned}
\| x_{n+1}- & p \|^{2} \\
\leq & \beta_{n}\left\|x_{n}-p\right\|^{2}+\left(1-\beta_{n}\right)\left\|y_{n}-p\right\|^{2} \\
& +2 \alpha_{n}\left\|\left(u+\gamma f\left(x_{n}\right)\right)-\bar{V} W_{n} y_{n}\right\|\left\|x_{n+1}-p\right\| \\
\leq & \beta_{n}\left\|x_{n}-p\right\|^{2}+\left(1-\beta_{n}\right) \\
& \times\left[\left\|x_{n}-p\right\|^{2}-\left\|\Lambda^{i-1} u_{n}-\Lambda^{i} u_{n}\right\|^{2}\right. \\
& \left.+2 \mu_{i}\left\|\Lambda^{i-1} u_{n}-\Lambda^{i} u_{n}\right\|\left\|B_{i} \Lambda^{i-1} u_{n}-B_{i} p\right\|\right] \\
& +2 \alpha_{n}\left\|\left(u+\gamma f\left(x_{n}\right)\right)-\bar{V} W_{n} y_{n}\right\|\left\|x_{n+1}-p\right\| \\
\leq & \left\|x_{n}-p\right\|^{2}-\left(1-\beta_{n}\right)\left\|\Lambda^{i-1} u_{n}-\Lambda^{i} u_{n}\right\|^{2} \\
& +2 \mu_{i}\left\|\Lambda^{i-1} u_{n}-\Lambda^{i} u_{n}\right\|\left\|B_{i} \Lambda^{i-1} u_{n}-B_{i} p\right\| \\
& +2 \alpha_{n}\left\|\left(u+\gamma f\left(x_{n}\right)\right)-\bar{V} W_{n} y_{n}\right\|\left\|x_{n+1}-p\right\| .
\end{aligned}
$$

So, it follows that

$$
\begin{aligned}
(1-\hat{d}) & \left\|\Lambda^{i-1} u_{n}-\Lambda^{i} u_{n}\right\|^{2} \\
\leq & \left(1-\beta_{n}\right)\left\|\Lambda^{i-1} u_{n}-\Lambda^{i} u_{n}\right\|^{2} \\
\leq & \left\|x_{n}-p\right\|^{2}-\left\|x_{n+1}-p\right\|^{2} \\
& +2 \mu_{i}\left\|\Lambda^{i-1} u_{n}-\Lambda^{i} u_{n}\right\|\left\|B_{i} \Lambda^{i-1} u_{n}-B_{i} p\right\| \\
& +2 \alpha_{n}\left\|\left(u+\gamma f\left(x_{n}\right)\right)-\bar{V} W_{n} y_{n}\right\|\left\|x_{n+1}-p\right\| \\
\leq & \left\|x_{n}-x_{n+1}\right\|\left(\left\|x_{n}-p\right\|+\left\|x_{n+1}-p\right\|\right) \\
& +2 \mu_{i}\left\|\Lambda^{i-1} u_{n}-\Lambda^{i} u_{n}\right\|\left\|B_{i} \Lambda^{i-1} u_{n}-B_{i} p\right\| \\
& +2 \alpha_{n}\left\|\left(u+\gamma f\left(x_{n}\right)\right)-\bar{V} W_{n} y_{n}\right\|\left\|x_{n+1}-p\right\| .
\end{aligned}
$$

Since $\lim _{n \rightarrow \infty} \alpha_{n}=0$ and $\lim _{n \rightarrow \infty}\left\|x_{n}-x_{n+1}\right\|=0$, from (96) and the boundedness of $\left\{x_{n}\right\},\left\{y_{n}\right\},\left\{W_{n} y_{n}\right\}$ and $\left\{f\left(x_{n}\right)\right\}$ we get

$$
\lim _{n \rightarrow \infty}\left\|\Lambda^{i-1} u_{n}-\Lambda^{i} u_{n}\right\|=0, \quad \forall i \in\{1,2, \ldots, N\}
$$

From (102) we get

$$
\begin{aligned}
\left\|u_{n}-z_{n}\right\|= & \left\|\Lambda^{0} u_{n}-\Lambda^{N} u_{n}\right\| \\
\leq & \left\|\Lambda^{0} u_{n}-\Lambda^{1} u_{n}\right\|+\left\|\Lambda^{1} u_{n}-\Lambda^{2} u_{n}\right\| \\
& +\cdots+\left\|\Lambda^{N-1} u_{n}-\Lambda^{N} u_{n}\right\|
\end{aligned}
$$$$
\longrightarrow 0 \quad \text { as } n \longrightarrow \infty \text {. }
$$

By (91) and (103), we have

$$
\begin{aligned}
\left\|x_{n}-z_{n}\right\| & \leq\left\|x_{n}-u_{n}\right\|+\left\|u_{n}-z_{n}\right\| \\
& \longrightarrow 0 \text { as } n \longrightarrow \infty .
\end{aligned}
$$

On the other hand, for simplicity, we write $\tilde{p}=T_{v_{2}}^{\Theta_{2}}(I-$ $\left.v_{2} A_{2}\right) p, v_{n}=T_{v_{2}}^{\Theta_{2}}\left(I-v_{2} A_{2}\right) z_{n}$ and $\widetilde{v}_{n}=G z_{n}=T_{v_{1}}^{\Theta_{1}}\left(I-v_{1} A_{1}\right) v_{n}$ for all $n \geq 1$. Then

$$
\begin{aligned}
p & =G p=T_{v_{1}}^{\Theta_{1}}\left(I-v_{1} A_{1}\right) \tilde{p} \\
& =T_{v_{1}}^{\Theta_{1}}\left(I-v_{1} A_{1}\right) T_{v_{2}}^{\Theta_{2}}\left(I-v_{2} A_{2}\right) p .
\end{aligned}
$$


We now show that $\lim _{n \rightarrow \infty}\left\|G z_{n}-z_{n}\right\|=0$, that is, $\lim _{n \rightarrow \infty}\left\|\widetilde{v}_{n}-z_{n}\right\|=0$. As a matter of fact, for $p \in \Omega$, it follows from (47), (54) and (55) that

$$
\begin{aligned}
\left\|y_{n}-p\right\|^{2} \leq & \delta_{n}\left\|G z_{n}-p\right\|^{2}+\left(1-\delta_{n}\right)\left\|W_{n} z_{n}-p\right\|^{2} \\
\leq & \delta_{n}\left\|\widetilde{v}_{n}-p\right\|^{2}+\left(1-\delta_{n}\right)\left\|z_{n}-p\right\|^{2} \\
\leq & \delta_{n}\left[\left\|v_{n}-\tilde{p}\right\|^{2}+v_{1}\left(v_{1}-2 \zeta_{1}\right)\left\|A_{1} v_{n}-A_{1} \tilde{p}\right\|^{2}\right] \\
& +\left(1-\delta_{n}\right)\left\|z_{n}-p\right\|^{2} \\
\leq & \delta_{n}\left[\left\|z_{n}-p\right\|^{2}+v_{2}\left(v_{2}-2 \zeta_{2}\right)\left\|A_{2} z_{n}-A_{2} p\right\|^{2}\right. \\
& \left.\quad+v_{1}\left(v_{1}-2 \zeta_{1}\right)\left\|A_{1} v_{n}-A_{1} \tilde{p}\right\|^{2}\right] \\
& +\left(1-\delta_{n}\right)\left\|z_{n}-p\right\|^{2} \\
= & \left\|z_{n}-p\right\|^{2}+\delta_{n}\left(v_{2}\left(v_{2}-2 \zeta_{2}\right)\left\|A_{2} z_{n}-A_{2} p\right\|^{2}\right. \\
\quad & \left.+v_{1}\left(v_{1}-2 \zeta_{1}\right)\left\|A_{1} v_{n}-A_{1} \tilde{p}\right\|^{2}\right) \\
\leq & \left\|x_{n}-p\right\|^{2} \\
& +\delta_{n}\left(v_{2}\left(v_{2}-2 \zeta_{2}\right)\left\|A_{2} z_{n}-A_{2} p\right\|^{2}\right. \\
& \left.+v_{1}\left(v_{1}-2 \zeta_{1}\right)\left\|A_{1} v_{n}-A_{1} \tilde{p}\right\|^{2}\right),
\end{aligned}
$$

which together with (73), implies that

$$
\begin{aligned}
\| x_{n+1}- & p \|^{2} \\
\leq & \beta_{n}\left\|x_{n}-p\right\|^{2}+\left(1-\beta_{n}\right)\left\|y_{n}-p\right\|^{2} \\
& +2 \alpha_{n}\left\|\left(u+\gamma f\left(x_{n}\right)\right)-\bar{V} W_{n} y_{n}\right\|\left\|x_{n+1}-p\right\| \\
\leq & \beta_{n}\left\|x_{n}-p\right\|^{2}+\left(1-\beta_{n}\right) \\
& \times\left[\left\|x_{n}-p\right\|^{2}\right. \\
& \quad+\delta_{n}\left(v_{2}\left(v_{2}-2 \zeta_{2}\right)\left\|A_{2} z_{n}-A_{2} p\right\|^{2}\right. \\
& \left.\left.\quad+v_{1}\left(v_{1}-2 \zeta_{1}\right)\left\|A_{1} v_{n}-A_{1} \tilde{p}\right\|^{2}\right)\right] \\
& +2 \alpha_{n}\left\|\left(u+\gamma f\left(x_{n}\right)\right)-\bar{V} W_{n} y_{n}\right\|\left\|x_{n+1}-p\right\| \\
= & \left\|x_{n}-p\right\|^{2} \quad \\
& +\left(1-\beta_{n}\right) \delta_{n}\left(v_{2}\left(v_{2}-2 \zeta_{2}\right)\left\|A_{2} z_{n}-A_{2} p\right\|^{2}\right. \\
& \left.\quad+v_{1}\left(v_{1}-2 \zeta_{1}\right)\left\|A_{1} v_{n}-A_{1} \tilde{p}\right\|^{2}\right) \\
& +2 \alpha_{n}\left\|\left(u+\gamma f\left(x_{n}\right)\right)-\bar{V} W_{n} y_{n}\right\|\left\|x_{n+1}-p\right\| .
\end{aligned}
$$

So, it follows that

$$
\begin{gathered}
(1-\hat{d}) \hat{c}\left(v_{2}\left(2 \zeta_{2}-v_{2}\right)\left\|A_{2} z_{n}-A_{2} p\right\|^{2}\right. \\
\left.+v_{1}\left(2 \zeta_{1}-v_{1}\right)\left\|A_{1} v_{n}-A_{1} \tilde{p}\right\|^{2}\right) \\
\leq\left(1-\beta_{n}\right) \delta_{n}\left(v_{2}\left(2 \zeta_{2}-v_{2}\right)\left\|A_{2} z_{n}-A_{2} p\right\|^{2}\right. \\
\left.+v_{1}\left(2 \zeta_{1}-v_{1}\right)\left\|A_{1} v_{n}-A_{1} \tilde{p}\right\|^{2}\right) \\
\leq\left\|x_{n}-p\right\|^{2}-\left\|x_{n+1}-p\right\|^{2} \\
+2 \alpha_{n}\left\|\left(u+\gamma f\left(x_{n}\right)\right)-\bar{V} W_{n} y_{n}\right\|\left\|x_{n+1}-p\right\| \\
\leq\left\|x_{n}-x_{n+1}\right\|\left(\left\|x_{n}-p\right\|+\left\|x_{n+1}-p\right\|\right) \\
+2 \alpha_{n}\left\|\left(u+\gamma f\left(x_{n}\right)\right)-\bar{V} W_{n} y_{n}\right\|\left\|x_{n+1}-p\right\| .
\end{gathered}
$$

Since $v_{k} \in\left(0,2 \zeta_{k}\right), k=1,2, \lim _{n \rightarrow \infty} \alpha_{n}=0$ and $\lim _{n \rightarrow \infty}\left\|x_{n}-x_{n+1}\right\|=0$, from the boundedness of $\left\{x_{n}\right\},\left\{y_{n}\right\},\left\{W_{n} y_{n}\right\}$ and $\left\{f\left(x_{n}\right)\right\}$ we get

$$
\lim _{n \rightarrow \infty}\left\|A_{2} z_{n}-A_{2} p\right\|=0, \quad \lim _{n \rightarrow \infty}\left\|A_{1} v_{n}-A_{1} \tilde{p}\right\|=0 .
$$

Also, in terms of the firm nonexpansivity of $T_{\gamma_{k}}^{\Theta_{k}}$ and the $\zeta_{k^{-}}$ inverse strong monotonicity of $A_{k}$ for $k=1$, 2, we obtain from $v_{k} \in\left(0,2 \zeta_{k}\right), k \in\{1,2\}$ and (54)-(55) that

$$
\begin{aligned}
& \left\|v_{n}-\tilde{p}\right\|^{2} \\
& =\left\|T_{v_{2}}^{\Theta_{2}}\left(I-v_{2} A_{2}\right) z_{n}-T_{v_{2}}^{\Theta_{2}}\left(I-v_{2} A_{2}\right) p\right\|^{2} \\
& \leq\left\langle\left(I-v_{2} A_{2}\right) z_{n}-\left(I-v_{2} A_{2}\right) p, v_{n}-\tilde{p}\right\rangle \\
& =\frac{1}{2}\left[\left\|\left(I-v_{2} A_{2}\right) z_{n}-\left(I-v_{2} A_{2}\right) p\right\|^{2}+\left\|v_{n}-\tilde{p}\right\|^{2}\right. \\
& \left.\quad-\left\|\left(I-v_{2} A_{2}\right) z_{n}-\left(I-v_{2} A_{2}\right) p-\left(v_{n}-\tilde{p}\right)\right\|^{2}\right] \\
& \leq \frac{1}{2}\left[\left\|z_{n}-p\right\|^{2}+\left\|v_{n}-\tilde{p}\right\|^{2}\right. \\
& \left.\quad-\left\|\left(z_{n}-v_{n}\right)-v_{2}\left(A_{2} z_{n}-A_{2} p\right)-(p-\widetilde{p})\right\|^{2}\right] \\
& \leq \frac{1}{2}\left[\left\|x_{n}-p\right\|^{2}+\left\|v_{n}-\widetilde{p}\right\|^{2}-\left\|\left(z_{n}-v_{n}\right)-(p-\tilde{p})\right\|^{2}\right. \\
& \quad+2 v_{2}\left\langle\left(z_{n}-v_{n}\right)-(p-\widetilde{p}), A_{2} z_{n}-A_{2} p\right\rangle \\
& \left.\quad-v_{2}^{2}\left\|A_{2} z_{n}-A_{2} p\right\|^{2}\right], \\
& \left\|\widetilde{v}_{n}-p\right\|^{2} \\
& =\left\|T_{v_{1}}^{\Theta_{1}}\left(I-v_{1} A_{1}\right) v_{n}-T_{v_{1}}^{\Theta_{1}}\left(I-v_{1} A_{1}\right) \tilde{p}\right\|^{2} \\
& \leq\left\langle\left(I-v_{1} A_{1}\right) v_{n}-\left(I-v_{1} A_{1}\right) \tilde{p}, \widetilde{v}_{n}-p\right\rangle \\
& =\frac{1}{2}\left[\left\|\left(I-v_{1} A_{1}\right) v_{n}-\left(I-v_{1} A_{1}\right) \tilde{p}\right\|^{2}+\left\|\widetilde{v}_{n}-p\right\|^{2}\right. \\
& \left.\quad-\left\|\left(I-v_{1} A_{1}\right) v_{n}-\left(I-v_{1} A_{1}\right) \widetilde{p}-\left(\widetilde{v}_{n}-p\right)\right\|^{2}\right]
\end{aligned}
$$




$$
\begin{gathered}
\leq \frac{1}{2}\left[\left\|v_{n}-\tilde{p}\right\|^{2}+\left\|\widetilde{v}_{n}-p\right\|^{2}-\left\|\left(v_{n}-\widetilde{v}_{n}\right)+(p-\tilde{p})\right\|^{2}\right. \\
+2 v_{1}\left\langle A_{1} v_{n}-A_{1} \tilde{p},\left(v_{n}-\widetilde{v}_{n}\right)+(p-\tilde{p})\right\rangle \\
\left.\quad-v_{1}^{2}\left\|A_{1} v_{n}-A_{1} \tilde{p}\right\|^{2}\right] \\
\leq \frac{1}{2}\left[\left\|x_{n}-p\right\|^{2}+\left\|\widetilde{v}_{n}-p\right\|^{2}-\left\|\left(v_{n}-\widetilde{v}_{n}\right)+(p-\tilde{p})\right\|^{2}\right. \\
\left.+2 v_{1}\left\langle A_{1} v_{n}-A_{1} \tilde{p},\left(v_{n}-\widetilde{v}_{n}\right)+(p-\widetilde{p})\right\rangle\right] .
\end{gathered}
$$

Thus, we have

$$
\begin{aligned}
\left\|v_{n}-\tilde{p}\right\|^{2} \leq & \left\|x_{n}-p\right\|^{2}-\left\|\left(z_{n}-v_{n}\right)-(p-\widetilde{p})\right\|^{2} \\
& +2 v_{2}\left\langle\left(z_{n}-v_{n}\right)-(p-\tilde{p}), A_{2} z_{n}-A_{2} p\right\rangle \\
& -v_{2}^{2}\left\|A_{2} z_{n}-A_{2} p\right\|^{2}, \\
\left\|\widetilde{v}_{n}-p\right\|^{2} \leq & \left\|x_{n}-p\right\|^{2}-\left\|\left(v_{n}-\widetilde{v}_{n}\right)+(p-\widetilde{p})\right\|^{2} \\
& +2 v_{1}\left\|A_{1} v_{n}-A_{1} \tilde{p}\right\|\left\|\left(v_{n}-\widetilde{v}_{n}\right)+(p-\tilde{p})\right\| .
\end{aligned}
$$

Consequently, from (47), (54), (55) and (111) it follows that

$$
\begin{aligned}
& \left\|y_{n}-p\right\|^{2} \\
& \leq \delta_{n}\left\|G z_{n}-p\right\|^{2}+\left(1-\delta_{n}\right)\left\|W_{n} z_{n}-p\right\|^{2} \\
& \leq \delta_{n}\left\|\widetilde{v}_{n}-p\right\|^{2}+\left(1-\delta_{n}\right)\left\|z_{n}-p\right\|^{2} \\
& \leq \delta_{n}\left\|v_{n}-\widetilde{p}\right\|^{2}+\left(1-\delta_{n}\right)\left\|z_{n}-p\right\|^{2} \\
& \leq \delta_{n}\left[\left\|x_{n}-p\right\|^{2}-\left\|\left(z_{n}-v_{n}\right)-(p-\widetilde{p})\right\|^{2}\right. \\
& \left.\quad+2 v_{2}\left\langle\left(z_{n}-v_{n}\right)-(p-\widetilde{p}), A_{2} z_{n}-A_{2} p\right\rangle\right] \\
& \quad+\left(1-\delta_{n}\right)\left\|x_{n}-p\right\|^{2} \\
& \leq\left\|x_{n}-p\right\|^{2}-\delta_{n}\left\|\left(z_{n}-v_{n}\right)-(p-\widetilde{p})\right\|^{2} \\
& \quad+2 v_{2}\left\|\left(z_{n}-v_{n}\right)-(p-\tilde{p})\right\|\left\|A_{2} z_{n}-A_{2} p\right\|,
\end{aligned}
$$

So, it follows that

$$
\begin{aligned}
(1-\widehat{d}) & \hat{c}\left\|\left(z_{n}-v_{n}\right)-(p-\tilde{p})\right\|^{2} \\
\leq & \left(1-\beta_{n}\right) \delta_{n}\left\|\left(z_{n}-v_{n}\right)-(p-\tilde{p})\right\|^{2} \\
\leq & \left\|x_{n}-p\right\|^{2}-\left\|x_{n+1}-p\right\|^{2} \\
& +2 v_{2}\left\|\left(z_{n}-v_{n}\right)-(p-\tilde{p})\right\|\left\|A_{2} z_{n}-A_{2} p\right\| \\
& +2 \alpha_{n}\left\|\left(u+\gamma f\left(x_{n}\right)\right)-\bar{V} W_{n} y_{n}\right\|\left\|x_{n+1}-p\right\| \\
\leq & \left\|x_{n}-x_{n+1}\right\|\left(\left\|x_{n}-p\right\|+\left\|x_{n+1}-p\right\|\right) \\
& +2 v_{2}\left\|\left(z_{n}-v_{n}\right)-(p-\tilde{p})\right\|\left\|A_{2} z_{n}-A_{2} p\right\| \\
& +2 \alpha_{n}\left\|\left(u+\gamma f\left(x_{n}\right)\right)-\bar{V} W_{n} y_{n}\right\|\left\|x_{n+1}-p\right\| .
\end{aligned}
$$

Since $\lim _{n \rightarrow \infty} \alpha_{n}=0$ and $\lim _{n \rightarrow \infty}\left\|x_{n}-x_{n+1}\right\|=0$, from (109) and the boundedness of $\left\{x_{n}\right\},\left\{y_{n}\right\},\left\{W_{n} y_{n}\right\}$ and $\left\{f\left(x_{n}\right)\right\}$ we get

$$
\lim _{n \rightarrow \infty}\left\|\left(z_{n}-v_{n}\right)-(p-\tilde{p})\right\|=0 .
$$

Furthermore, from (47), (54) and (112) it follows that

$$
\begin{aligned}
\left\|y_{n}-p\right\|^{2} \leq & \delta_{n}\left\|G z_{n}-p\right\|^{2}+\left(1-\delta_{n}\right)\left\|W_{n} z_{n}-p\right\|^{2} \\
\leq & \delta_{n}\left\|\widetilde{v}_{n}-p\right\|^{2}+\left(1-\delta_{n}\right)\left\|z_{n}-p\right\|^{2} \\
\leq & \delta_{n}\left[\left\|x_{n}-p\right\|^{2}-\left\|\left(v_{n}-\widetilde{v}_{n}\right)+(p-\widetilde{p})\right\|^{2}\right. \\
& \left.\quad+2 v_{1}\left\|A_{1} v_{n}-A_{1} \tilde{p}\right\|\left\|\left(v_{n}-\widetilde{v}_{n}\right)+(p-\widetilde{p})\right\|\right] \\
& +\left(1-\delta_{n}\right)\left\|x_{n}-p\right\|^{2} \\
\leq & \left\|x_{n}-p\right\|^{2}-\delta_{n}\left\|\left(v_{n}-\widetilde{v}_{n}\right)+(p-\widetilde{p})\right\|^{2} \\
& +2 v_{1}\left\|A_{1} v_{n}-A_{1} \tilde{p}\right\|\left\|\left(v_{n}-\widetilde{v}_{n}\right)+(p-\widetilde{p})\right\|,
\end{aligned}
$$


which together with (73), implies that

$$
\begin{aligned}
\| x_{n+1}- & p \|^{2} \\
\leq & \beta_{n}\left\|x_{n}-p\right\|^{2}+\left(1-\beta_{n}\right)\left\|y_{n}-p\right\|^{2} \\
& +2 \alpha_{n}\left\|\left(u+\gamma f\left(x_{n}\right)\right)-\bar{V} W_{n} y_{n}\right\|\left\|x_{n+1}-p\right\| \\
\leq & \beta_{n}\left\|x_{n}-p\right\|^{2}+\left(1-\beta_{n}\right) \\
& \times\left[\left\|x_{n}-p\right\|^{2}-\delta_{n}\left\|\left(v_{n}-\widetilde{v}_{n}\right)+(p-\widetilde{p})\right\|^{2}\right. \\
& \left.\quad+2 v_{1}\left\|A_{1} v_{n}-A_{1} \widetilde{p}\right\|\left\|\left(v_{n}-\widetilde{v}_{n}\right)+(p-\widetilde{p})\right\|\right] \\
& +2 \alpha_{n}\left\|\left(u+\gamma f\left(x_{n}\right)\right)-\bar{V} W_{n} y_{n}\right\|\left\|x_{n+1}-p\right\| \\
\leq & \left\|x_{n}-p\right\|^{2}-\left(1-\beta_{n}\right) \delta_{n}\left\|\left(v_{n}-\widetilde{v}_{n}\right)+(p-\widetilde{p})\right\|^{2} \\
& +2 v_{1}\left\|A_{1} v_{n}-A_{1} \tilde{p}\right\|\left\|\left(v_{n}-\widetilde{v}_{n}\right)+(p-\widetilde{p})\right\| \\
& +2 \alpha_{n}\left\|\left(u+\gamma f\left(x_{n}\right)\right)-\bar{V} W_{n} y_{n}\right\|\left\|x_{n+1}-p\right\| .
\end{aligned}
$$

So, it follows that

$$
\begin{aligned}
(1-\hat{d}) & \hat{c}\left\|\left(v_{n}-\widetilde{v}_{n}\right)+(p-\tilde{p})\right\|^{2} \\
\leq & \left(1-\beta_{n}\right) \delta_{n}\left\|\left(v_{n}-\widetilde{v}_{n}\right)+(p-\tilde{p})\right\|^{2} \\
\leq & \left\|x_{n}-p\right\|^{2}-\left\|x_{n+1}-p\right\|^{2} \\
& +2 v_{1}\left\|A_{1} v_{n}-A_{1} \tilde{p}\right\|\left\|\left(v_{n}-\widetilde{v}_{n}\right)+(p-\tilde{p})\right\| \\
& +2 \alpha_{n}\left\|\left(u+\gamma f\left(x_{n}\right)\right)-\bar{V} W_{n} y_{n}\right\|\left\|x_{n+1}-p\right\| \\
\leq & \left\|x_{n}-x_{n+1}\right\|\left(\left\|x_{n}-p\right\|+\left\|x_{n+1}-p\right\|\right) \\
& +2 v_{1}\left\|A_{1} v_{n}-A_{1} \tilde{p}\right\|\left\|\left(v_{n}-\widetilde{v}_{n}\right)+(p-\tilde{p})\right\| \\
& +2 \alpha_{n}\left\|\left(u+\gamma f\left(x_{n}\right)\right)-\bar{V} W_{n} y_{n}\right\|\left\|x_{n+1}-p\right\| .
\end{aligned}
$$

Since $\lim _{n \rightarrow \infty} \alpha_{n}=0$ and $\lim _{n \rightarrow \infty}\left\|x_{n}-x_{n+1}\right\|=0$, from (109) and the boundedness of $\left\{x_{n}\right\},\left\{y_{n}\right\},\left\{W_{n} y_{n}\right\}$ and $\left\{f\left(x_{n}\right)\right\}$ we get

$$
\lim _{n \rightarrow \infty}\left\|\left(v_{n}-\widetilde{v}_{n}\right)+(p-\tilde{p})\right\|=0 .
$$

Note that

$$
\left\|z_{n}-\widetilde{v}_{n}\right\| \leq\left\|\left(z_{n}-v_{n}\right)-(p-\tilde{p})\right\|+\left\|\left(v_{n}-\widetilde{v}_{n}\right)+(p-\tilde{p})\right\| .
$$

Hence from (116) and (120) we get

$$
\lim _{n \rightarrow \infty}\left\|z_{n}-\widetilde{v}_{n}\right\|=\lim _{n \rightarrow \infty}\left\|z_{n}-G z_{n}\right\|=0,
$$

which together with (79), implies that

$$
\begin{aligned}
\left\|z_{n}-W_{n} z_{n}\right\| & \leq\left\|z_{n}-G z_{n}\right\|+\left\|G z_{n}-W_{n} z_{n}\right\| \\
& \longrightarrow 0 \text { as } n \longrightarrow \infty .
\end{aligned}
$$

Also, observe that

$$
\left\|z_{n}-W z_{n}\right\| \leq\left\|z_{n}-W_{n} z_{n}\right\|+\left\|W_{n} z_{n}-W z_{n}\right\| .
$$

From (122), Remark 10 and the boundedness of $\left\{z_{n}\right\}$ we immediately obtain

$$
\lim _{n \rightarrow \infty}\left\|z_{n}-W z_{n}\right\|=0
$$

Step 5. We show that

$$
\limsup _{n \rightarrow \infty}\left\langle u+(\gamma f-\bar{V}) x^{*}, x_{n}-x^{*}\right\rangle \leq 0,
$$

where $x^{*}$ is a solution of (OP2).

Indeed, we note that $V$ is a $\bar{\gamma}$-strongly positive bounded linear operator and $f: H \rightarrow H$ is an $l$-Lipschitzian mapping with $\gamma l<(1+\mu) \bar{\gamma}$. It is clear that

$$
\begin{gathered}
\langle(\bar{V} x-(u+\gamma f(x)))-(\bar{V} y-(u+\gamma f(y))), x-y\rangle \\
\geq((1+\mu) \bar{\gamma}-\gamma l)\|x-y\|^{2}, \quad \forall x, y \in H .
\end{gathered}
$$

Hence we deduce that $\bar{V} x-(u+\gamma f(x))$ is $((1+\mu) \bar{\gamma}-\gamma l)$-strongly monotone. In the meantime, it is easy to see that $\bar{V} x-(u+$ $\gamma f(x))$ is $(\|\bar{V}\|+\gamma l)$-Lipschitzian with constant $\|\bar{V}\|+\gamma l>0$. Thus, there exists a unique solution $x^{*}$ in $\Omega$ to the VIP

$$
\left\langle u+(\gamma f-\bar{V}) x^{*}, u-x^{*}\right\rangle \leq 0, \quad \forall u \in \Omega
$$

Equivalently, $x^{*} \in \Omega$ solves (OP2) (due to Lemma 20).

First, we observe that there exists a subsequence $\left\{x_{n_{i}}\right\}$ of $\left\{x_{n}\right\}$ such that

$$
\begin{aligned}
\limsup _{n \rightarrow \infty} & \left\langle u+(\gamma f-\bar{V}) x^{*}, x_{n}-x^{*}\right\rangle \\
& =\lim _{i \rightarrow \infty}\left\langle u+(\gamma f-\bar{V}) x^{*}, x_{n_{i}}-x^{*}\right\rangle .
\end{aligned}
$$

Since $\left\{x_{n_{i}}\right\}$ is bounded, there exists a subsequence $\left\{x_{n_{i_{j}}}\right\}$ of $\left\{x_{n_{i}}\right\}$ which converges weakly to some $w$. Without loss of generality, we may assume that $x_{n_{i}} \rightarrow w$. From (91) and (102)(104), we have that $u_{n_{i}} \rightarrow w, \Lambda^{m} u_{n_{i}} \rightarrow w$ and $z_{n_{i}} \rightarrow w$, where $m \in\{1,2, \ldots, N\}$. By (122) and (125) we have that $\left\|G z_{n}-z_{n}\right\| \rightarrow 0$ and $\left\|W z_{n}-z_{n}\right\| \rightarrow 0$ as $n \rightarrow \infty$. Utilizing the similar arguments to those of (55), we know that $G$ is nonexpansive. Hence, by Lemma 12 we obtain $w \in$ $\operatorname{Fix}(G)=\operatorname{SGEP}(G)$ and $w \in \operatorname{Fix}(W)=\cap_{n=1}^{\infty} \operatorname{Fix}\left(T_{n}\right)$ (due to Lemma 11). Next, we prove that $w \in \cap_{m=1}^{N} I\left(B_{m}, R_{m}\right)$. As a matter of fact, since $B_{m}$ is $\eta_{m}$-inverse strongly monotone, $B_{m}$ is a monotone and Lipschitz continuous mapping. It follows from Lemma 19 that $R_{m}+B_{m}$ is maximal monotone. Let $(v, g) \in G\left(R_{m}+B_{m}\right)$, that is, $g-B_{m} v \in R_{m} v$. Again, since $\Lambda^{m} u_{n}=J_{R_{m}, \mu_{m}}\left(I-\mu_{m} B_{m}\right) \Lambda^{m-1} u_{n}, n \geq 1, m \in\{1,2, \ldots, N\}$, we have

$$
\Lambda^{m-1} u_{n}-\mu_{m} B_{m} \Lambda^{m-1} u_{n} \in\left(I+\mu_{m} R_{m}\right) \Lambda^{m} u_{n} .
$$

that is,

$$
\frac{1}{\mu_{m}}\left(\Lambda^{m-1} u_{n}-\Lambda^{m} u_{n}-\mu_{m} B_{m} \Lambda^{m-1} u_{n}\right) \in R_{m} \Lambda^{m} u_{n} .
$$


In terms of the monotonicity of $R_{m}$, we get

$$
\begin{aligned}
& \left\langle v-\Lambda^{m} u_{n}, g-B_{m} v\right. \\
& \left.\quad-\frac{1}{\mu_{m}}\left(\Lambda^{m-1} u_{n}-\Lambda^{m} u_{n}-\mu_{m} B_{m} \Lambda^{m-1} u_{n}\right)\right\rangle \\
& \quad \geq 0
\end{aligned}
$$

and hence

$$
\begin{aligned}
& \left\langle v-\Lambda^{m} u_{n}, g\right\rangle \\
& \geq\left\langle v-\Lambda^{m} u_{n}, B_{m} v\right. \\
& \left.\quad+\frac{1}{\mu_{m}}\left(\Lambda^{m-1} u_{n}-\Lambda^{m} u_{n}-\mu_{m} B_{m} \Lambda^{m-1} u_{n}\right)\right\rangle \\
& =\left\langle v-\Lambda^{m} u_{n}, B_{m} v-B_{m} \Lambda^{m} u_{n}+B_{m} \Lambda^{m} u_{n}-B_{m} \Lambda^{m-1} u_{n}\right. \\
& \left.\quad+\frac{1}{\mu_{m}}\left(\Lambda^{m-1} u_{n}-\Lambda^{m} u_{n}\right)\right\rangle \\
& \geq\left\langle v-\Lambda^{m} u_{n}, B_{m} \Lambda^{m} u_{n}-B_{m} \Lambda^{m-1} u_{n}\right\rangle \\
& \quad\left\langle v-\Lambda^{m} u_{n}, \frac{1}{\mu_{m}}\left(\Lambda^{m-1} u_{n}-\Lambda^{m} u_{n}\right)\right\rangle
\end{aligned}
$$

In particular,

$$
\begin{aligned}
\left\langle v-\Lambda^{m} u_{n_{i}}, g\right\rangle \geq & \left\langle v-\Lambda^{m} u_{n_{i}}, B_{m} \Lambda^{m} u_{n_{i}}-B_{m} \Lambda^{m-1} u_{n_{i}}\right\rangle \\
& +\left\langle v-\Lambda^{m} u_{n_{i}}, \frac{1}{\mu_{m}}\left(\Lambda^{m-1} u_{n_{i}}-\Lambda^{m} u_{n_{i}}\right)\right\rangle .
\end{aligned}
$$

Since $\left\|\Lambda^{m} u_{n}-\Lambda^{m-1} u_{n}\right\| \rightarrow 0$ (due to (102)) and $\| B_{m} \Lambda^{m} u_{n}-$ $B_{m} \Lambda^{m-1} u_{n} \| \rightarrow 0$ (due to the Lipschitz continuity of $B_{m}$ ), we conclude from $\Lambda^{m} u_{n_{i}} \rightarrow w$ and $\mu_{m} \in\left(0,2 \eta_{m}\right), m \in$ $\{1,2, \ldots, N\}$ that

$$
\lim _{i \rightarrow \infty}\left\langle v-\Lambda^{m} u_{n_{i}}, g\right\rangle=\langle v-w, g\rangle \geq 0
$$

It follows from the maximal monotonicity of $B_{m}+R_{m}$ that $0 \in\left(R_{m}+B_{m}\right) w$, that is, $w \in I\left(B_{m}, R_{m}\right)$. Therefore, $w \in$ $\cap_{m=1}^{N} I\left(B_{m}, R_{m}\right)$.

Next, we show that $w \in \operatorname{GMEP}(\Theta, \varphi, A)$. In fact, from $u_{n}=S_{r_{n}}^{(\Theta, \varphi)}\left(I-r_{n} A\right) x_{n}$, we know that

$$
\begin{aligned}
\Theta\left(u_{n}, y\right)+\varphi(y)-\varphi\left(u_{n}\right)+\left\langle A x_{n}, y-u_{n}\right\rangle \\
\quad+\frac{1}{r_{n}}\left\langle K^{\prime}\left(u_{n}\right)-K^{\prime}\left(x_{n}\right), y-u_{n}\right\rangle \geq 0, \quad \forall y \in C .
\end{aligned}
$$

From (H2) it follows that

$$
\begin{aligned}
& \varphi(y)-\varphi\left(u_{n}\right)+\left\langle A x_{n}, y-u_{n}\right\rangle \\
&+\frac{1}{r_{n}}\left\langle K^{\prime}\left(u_{n}\right)-K^{\prime}\left(x_{n}\right), y-u_{n}\right\rangle \geq \Theta\left(y, u_{n}\right), \\
& \forall y \in C .
\end{aligned}
$$

Replacing $n$ by $n_{i}$, we have

$$
\begin{aligned}
\varphi(y) & -\varphi\left(u_{n_{i}}\right)+\left\langle A x_{n_{i}}, y-u_{n_{i}}\right\rangle \\
+ & \left\langle\frac{K^{\prime}\left(u_{n_{i}}\right)-K^{\prime}\left(x_{n_{i}}\right)}{r_{n_{i}}}, y-u_{n_{i}}\right\rangle \geq \Theta\left(y, u_{n_{i}}\right),
\end{aligned}
$$

Put $u_{t}=t y+(1-t) w$ for all $t \in(0,1]$ and $y \in C$. Then, from (138) we have

$$
\begin{aligned}
\left\langle u_{t}-\right. & \left.u_{n_{i}}, A u_{t}\right\rangle \\
\geq & \left\langle u_{t}-u_{n_{i}}, A u_{t}\right\rangle-\varphi\left(u_{t}\right) \\
& +\varphi\left(u_{n_{i}}\right)-\left\langle u_{t}-u_{n_{i}}, A x_{n_{i}}\right\rangle \\
& -\left\langle\frac{K^{\prime}\left(u_{n_{i}}\right)-K^{\prime}\left(x_{n_{i}}\right)}{r_{n_{i}}}, u_{t}-u_{n_{i}}\right\rangle+\Theta\left(u_{t}, u_{n_{i}}\right) \\
\geq & \left\langle u_{t}-u_{n_{i}}, A u_{t}-A u_{n_{i}}\right\rangle \\
& +\left\langle u_{t}-u_{n_{i}}, A u_{n_{i}}-A x_{n_{i}}\right\rangle-\varphi\left(u_{t}\right)+\varphi\left(u_{n_{i}}\right) \\
& -\left\langle\frac{K^{\prime}\left(u_{n_{i}}\right)-K^{\prime}\left(x_{n_{i}}\right)}{r_{n_{i}}}, u_{t}-u_{n_{i}}\right\rangle+\Theta\left(u_{t}, u_{n_{i}}\right) .
\end{aligned}
$$

Since $\left\|u_{n_{i}}-x_{n_{i}}\right\| \rightarrow 0$ as $i \rightarrow \infty$, we deduce from the Lipschitz continuity of $A$ and $K^{\prime}$ that $\left\|A u_{n_{i}}-A x_{n_{i}}\right\| \rightarrow 0$ and $\left\|K^{\prime}\left(u_{n_{i}}\right)-K^{\prime}\left(x_{n_{i}}\right)\right\| \rightarrow 0$ as $i \rightarrow \infty$. Further, from the monotonicity of $A$, we have $\left\langle u_{t}-u_{n_{i}}, A u_{t}-A u_{n_{i}}\right\rangle \geq 0$. So, from (H4), the weakly lower semicontinuity of $\varphi,\left(K^{\prime}\left(u_{n_{i}}\right)-\right.$ $\left.K^{\prime}\left(x_{n_{i}}\right)\right) / r_{n_{i}} \rightarrow 0$ and $u_{n_{i}} \rightarrow w$, we have

$$
\left\langle u_{t}-w, A u_{t}\right\rangle \geq-\varphi\left(u_{t}\right)+\varphi(w)+\Theta\left(u_{t}, w\right), \quad \text { as } i \longrightarrow \infty .
$$


From (H1), (H4) and (140) we also have

$$
\begin{aligned}
0= & \Theta\left(u_{t}, u_{t}\right)+\varphi\left(u_{t}\right)-\varphi\left(u_{t}\right) \\
\leq & t \Theta\left(u_{t}, y\right)+(1-t) \Theta\left(u_{t}, w\right)+t \varphi(y) \\
& +(1-t) \varphi(w)-\varphi\left(u_{t}\right) \\
= & t\left[\Theta\left(u_{t}, y\right)+\varphi(y)-\varphi\left(u_{t}\right)\right] \\
& +(1-t)\left[\Theta\left(u_{t}, w\right)+\varphi(w)-\varphi(w)-\varphi\left(u_{t}\right)\right] \\
\leq & t\left[\Theta\left(u_{t}, y\right)+\varphi(y)-\varphi\left(u_{t}\right)\right]+(1-t)\left\langle u_{t}-w, A u_{t}\right\rangle \\
= & t\left[\Theta\left(u_{t}, y\right)+\varphi(y)-\varphi\left(u_{t}\right)\right]+(1-t) t\left\langle y-w, A u_{t}\right\rangle,
\end{aligned}
$$

and hence

$$
0 \leq \Theta\left(u_{t}, y\right)+\varphi(y)-\varphi\left(u_{t}\right)+(1-t)\left\langle y-w, A u_{t}\right\rangle .
$$

Letting $t \rightarrow 0$, we have, for each $y \in C$,

$$
0 \leq \Theta(w, y)+\varphi(y)-\varphi(w)+\langle A w, y-w\rangle
$$

This implies that $w \in \operatorname{GMEP}(\Theta, \varphi, A)$. Therefore, $w \in$ $\cap_{n=1}^{\infty} \operatorname{Fix}\left(T_{n}\right) \cap \operatorname{GMEP}(\Theta, \varphi, A) \cap \operatorname{SGEP}(G) \cap \cap_{i=1}^{N} I\left(B_{i}, R_{i}\right):=\Omega$. This shows that $\omega_{w}\left(x_{n}\right) \subset \Omega$. Consequently, from (128) and (129) we have

$$
\begin{gathered}
\limsup _{n \rightarrow \infty}\left\langle u+(\gamma f-\bar{V}) x^{*}, x_{n}-x^{*}\right\rangle \\
=\left\langle u+(\gamma f-\bar{V}) x^{*}, w-x^{*}\right\rangle \leq 0 .
\end{gathered}
$$

Step 6. Finally, we show that $x_{n} \rightarrow x^{*} \in \Omega$ as $n \rightarrow \infty$. Indeed, from (47) and (54), we have

$$
\begin{aligned}
\left\|y_{n}-x^{*}\right\|^{2} & \leq \delta_{n}\left\|G z_{n}-x^{*}\right\|^{2}+\left(1-\delta_{n}\right)\left\|W_{n} z_{n}-x^{*}\right\|^{2} \\
& \leq\left\|z_{n}-x^{*}\right\|^{2} \leq\left\|x_{n}-x^{*}\right\|^{2} .
\end{aligned}
$$

In terms of Lemma 6 we have

$$
\begin{aligned}
& \left\|x_{n+1}-x^{*}\right\|^{2} \\
& =\| \alpha_{n}\left(u+\gamma f\left(x_{n}\right)-\bar{V} x^{*}\right)+\beta_{n}\left(x_{n}-x^{*}\right) \\
& \quad+\left(\left(1-\beta_{n}\right) I-\alpha_{n} \bar{V}\right)\left(W_{n} y_{n}-x^{*}\right) \|^{2}
\end{aligned}
$$

$$
\begin{aligned}
\leq & \left\|\beta_{n}\left(x_{n}-x^{*}\right)+\left(\left(1-\beta_{n}\right) I-\alpha_{n} \bar{V}\right)\left(W_{n} y_{n}-x^{*}\right)\right\|^{2} \\
& +2 \alpha_{n}\left\langle u+\gamma f\left(x_{n}\right)-\bar{V} x^{*}, x_{n+1}-x^{*}\right\rangle \\
\leq & {\left[\left\|\left(\left(1-\beta_{n}\right) I-\alpha_{n} \bar{V}\right)\left(W_{n} y_{n}-x^{*}\right)\right\|+\beta_{n}\left\|x_{n}-x^{*}\right\|\right]^{2} 2 } \\
& +2 \alpha_{n} \gamma\left\langle f\left(x_{n}\right)-f\left(x^{*}\right), x_{n+1}-x^{*}\right\rangle \\
& +2 \alpha_{n}\left\langle u+\gamma f\left(x^{*}\right)-\bar{V} x^{*}, x_{n+1}-x^{*}\right\rangle \\
\leq & {\left[\left(1-\beta_{n}-\alpha_{n}(1+\mu) \bar{\gamma}\right)\left\|y_{n}-x^{*}\right\|+\beta_{n}\left\|x_{n}-x^{*}\right\|\right]^{2} } \\
& +2 \alpha_{n} \gamma l\left\|x_{n}-x^{*}\right\|\left\|x_{n+1}-x^{*}\right\| \\
& +2 \alpha_{n}\left\langle u+\gamma f\left(x^{*}\right)-\bar{V} x^{*}, x_{n+1}-x^{*}\right\rangle \\
\leq & \left(1-\alpha_{n}(1+\mu) \bar{\gamma}\right)^{2}\left\|x_{n}-x^{*}\right\|^{2} \\
& +\alpha_{n} \gamma l\left(\left\|x_{n}-x^{*}\right\|^{2}+\left\|x_{n+1}-x^{*}\right\|^{2}\right) \\
& +2 \alpha_{n}\left\langle u+\gamma f\left(x^{*}\right)-\bar{V} x^{*}, x_{n+1}-x^{*}\right\rangle,
\end{aligned}
$$

which leads to

$$
\left\|x_{n+1}-x^{*}\right\|^{2}
$$

$$
\begin{aligned}
& \leq \frac{1-2 \alpha_{n}(1+\mu) \bar{\gamma}+\alpha_{n}^{2}(1+\mu)^{2} \bar{\gamma}^{2}+\alpha_{n} \gamma l}{1-\alpha_{n} \gamma l} \\
& \times\left\|x_{n}-x^{*}\right\|^{2} \\
& +\frac{2 \alpha_{n}}{1-\alpha_{n} \gamma l}\left\langle u+\gamma f\left(x^{*}\right)-\bar{V} x^{*}, x_{n+1}-x^{*}\right\rangle \\
& =\left[1-\frac{2((1+\mu) \bar{\gamma}-\gamma l) \alpha_{n}}{1-\alpha_{n} \gamma l}\right]\left\|x_{n}-x^{*}\right\|^{2} \\
& +\frac{\left((1+\mu) \alpha_{n} \bar{\gamma}\right)^{2}}{1-\alpha_{n} \gamma l}\left\|x_{n}-x^{*}\right\|^{2} \\
& +\frac{2 \alpha_{n}}{1-\alpha_{n} \gamma l}\left\langle u+\gamma f\left(x^{*}\right)-\bar{V} x^{*}, x_{n+1}-x^{*}\right\rangle \\
& \leq\left[1-\frac{2((1+\mu) \bar{\gamma}-\gamma l) \alpha_{n}}{1-\alpha_{n} \gamma l}\right]\left\|x_{n}-x^{*}\right\|^{2} \\
& +\frac{2((1+\mu) \bar{\gamma}-\gamma l) \alpha_{n}}{1-\alpha_{n} \gamma l} \\
& \times\left\{\frac{\left(\alpha_{n}(1+\mu)^{2} \bar{\gamma}^{2}\right) M_{0}}{2((1+\mu) \bar{\gamma}-\gamma l)}+\frac{1}{(1+\mu) \bar{\gamma}-\gamma l}\right. \\
& \left.\times\left\langle u+\gamma f\left(x^{*}\right)-\bar{V} x^{*}, x_{n+1}-x^{*}\right\rangle\right\} \\
& =\left(1-\gamma_{n}\right)\left\|x_{n}-x^{*}\right\|^{2}+\sigma_{n} \gamma_{n}
\end{aligned}
$$


where $M_{0}=\sup \left\{\left\|x_{n}-x^{*}\right\|^{2}: n \geq 1\right\}, \gamma_{n}=2((1+\mu) \bar{\gamma}-$ $\gamma l) \alpha_{n} /\left(1-\alpha_{n} \gamma l\right)$ and $\sigma_{n}=\left(\left(\alpha_{n}(1+\mu)^{2} \bar{\gamma}^{2}\right) M_{0} / 2((1+\mu) \bar{\gamma}-\right.$ $\gamma l))+(1 /((1+\mu) \bar{\gamma}-\gamma l))\left\langle u+\gamma f\left(x^{*}\right)-\bar{V} x^{*}, x_{n+1}-x^{*}\right\rangle$. It is easy to see that $\sum_{n=1}^{\infty} \gamma_{n}=\infty$ and $\limsup _{n \rightarrow \infty} \sigma_{n} \leq 0$. Hence by Lemma 15 , we infer that the sequence $\left\{x_{n}\right\}$ converges strongly to $x^{*}$. This completes the proof.

Corollary 22. Let $C$ be a nonempty closed convex subset of a real Hilbert space $H$. Let $N$ be an integer. Let $\Theta$ be a bifunction from $\mathrm{C} \times \mathrm{C}$ to $\mathbf{R}$ satisfying $(\mathrm{H} 1)-(\mathrm{H} 4)$ and $\varphi: C \rightarrow \mathbf{R}$ be a lower semicontinuous and convex functional. Let $R_{i}: C \rightarrow 2^{H}$ be a maximal monotone mapping and let $A: H \rightarrow H$ and $B_{i}: C \rightarrow H$ be $\zeta$-inverse strongly monotone and $\eta_{i}$-inverse strongly monotone, respectively, where $i \in\{1,2, \ldots, N\}$. Let $\left\{T_{n}\right\}_{n=1}^{\infty}$ be a sequence of nonexpansive mappings on $H$ and $\left\{\lambda_{n}\right\}$ be a sequence in $(0, b]$ for some $b \in(0,1)$. Let $V$ be a $\bar{\gamma}$-strongly positive bounded linear operator and $f: H \rightarrow H$ be an l-Lipschitzian mapping with $\gamma l<(1+\mu) \bar{\gamma}$. Let $W_{n}$ be the $W$-mapping defined by (9). Assume that $\Omega:=\cap_{n=1}^{\infty} \operatorname{Fix}\left(T_{n}\right) \cap$ $\operatorname{GMEP}(\Theta, \varphi, A) \cap \cap_{i=1}^{N} I\left(B_{i}, R_{i}\right) \neq \emptyset$. Let $\left\{\alpha_{n}\right\},\left\{\beta_{n}\right\}$ and $\left\{\delta_{n}\right\}$ be three sequences in $[0,1]$. Assume that:

(i) $K: H \rightarrow \mathbf{R}$ is strongly convex with constant $\sigma>0$ and its derivative $K^{\prime}$ is Lipschitz continuous with constant $v>0$ such that the function $x \mapsto\left\langle y-x, K^{\prime}(x)\right\rangle$ is weakly upper semicontinuous for each $y \in H$;

(ii) for each $x \in H$, there exist a bounded subset $D_{x} \subset C$ and $z_{x} \in C$ such that for any $y \notin D_{x}$,

$$
\begin{aligned}
\Theta\left(y, z_{x}\right)+\varphi\left(z_{x}\right)-\varphi(y) \\
+\frac{1}{r}\left\langle K^{\prime}(y)-K^{\prime}(x), z_{x}-y\right\rangle<0 ;
\end{aligned}
$$

(iii) $\lim _{n \rightarrow \infty} \alpha_{n}=0, \sum_{n=1}^{\infty} \alpha_{n}=\infty, 0<\liminf _{n \rightarrow \infty} \beta_{n} \leq$ $\limsup \sup _{n \rightarrow \infty} \beta_{n}<1$ and $0<\liminf _{n \rightarrow \infty} \delta_{n} \leq$ $\lim \sup _{n \rightarrow \infty} \delta_{n}<1$

(iv) $\mu_{i} \in\left(0,2 \eta_{i}\right), i \in\{1,2, \ldots, N\}$, and $\left\{r_{n}\right\} \subset[0,2 \zeta]$ satisfies $0<\liminf _{n \rightarrow \infty} r_{n} \leq \limsup _{n \rightarrow \infty} r_{n}<2 \zeta$;

(v) $\lim _{n \rightarrow \infty}\left(\left|\delta_{n+1}-\delta_{n}\right|+\left|r_{n+1}-r_{n}\right|\right)=0$.

Given $x_{1} \in H$ arbitrarily, then the sequence $\left\{x_{n}\right\}$ generated iteratively by

$$
\begin{gathered}
u_{n}=S_{r_{n}}^{(\Theta, \varphi)}\left(I-r_{n} A\right) x_{n}, \\
z_{n}=J_{R_{N}, \mu_{N}}\left(I-\mu_{N} B_{N}\right) J_{R_{N-1}, \mu_{N-1}} \\
\times\left(I-\mu_{N-1} B_{N-1}\right) \cdots J_{R_{1}, \mu_{1}}\left(I-\mu_{1} B_{1}\right) u_{n}, \\
y_{n}=\delta_{n} z_{n}+\left(1-\delta_{n}\right) W_{n} z_{n}, \\
x_{n+1}=\alpha_{n}\left(u+\gamma f\left(x_{n}\right)\right)+\beta_{n} x_{n} \\
+\left(\left(1-\beta_{n}\right) I-\alpha_{n}(I+\mu V)\right) W_{n} y_{n}, \quad \forall n \geq 1,
\end{gathered}
$$

converges strongly to $x^{*} \in \Omega$ which solves the following optimization problem provided $S_{r}^{(\Theta, \varphi)}$ is firmly nonexpansive:

$$
\min _{x \in \Omega} \frac{\mu}{2}\langle A x, x\rangle+\frac{1}{2}\|x-u\|^{2}-h(x),
$$

where $h$ is the potential function of $\gamma f$.
Proof. In Theorem 21, putting $\Theta_{1} \equiv \Theta_{2} \equiv 0$ and $A_{1} \equiv A_{2} \equiv 0$, we get $G z_{n}=z_{n}$ and $\operatorname{SGEP}(G)=C$. Utilizing Theorem 21 we derive the desired result.

Corollary 23. Let $C$ be a nonempty closed convex subset of a real Hilbert space $H$. Let $\Theta, \Theta_{1}, \Theta_{2}$ be three bifunctions from $C \times C$ to $\mathbf{R}$ satisfying $(\mathrm{H1})-(\mathrm{H} 4)$ and $\varphi: C \rightarrow \mathbf{R}$ be a lower semicontinuous and convex functional. Let $R_{i}: C \rightarrow 2^{H}$ be a maximal monotone mapping and let $A, A_{k}: H \rightarrow$ $H$ and $B_{i}: C \rightarrow H$ be $\zeta$-inverse strongly monotone, $\zeta_{k^{-}}$ inverse strongly monotone and $\eta_{i}$-inverse strongly monotone, respectively, for $i=1,2$ and $k=1,2$. Let $\left\{T_{n}\right\}_{n=1}^{\infty}$ be a sequence of nonexpansive mappings on $H$ and $\left\{\lambda_{n}\right\}$ be a sequence in $(0, b]$ for some $b \in(0,1)$. Let $V$ be a $\bar{\gamma}$-strongly positive bounded linear operator and $f: H \rightarrow H$ be an l-Lipschitzian mapping with $\gamma l<(1+\mu) \bar{\gamma}$. Let $W_{n}$ be the $W$-mapping defined by (9). Assume that $\Omega:=\cap_{n=1}^{\infty} \operatorname{Fix}\left(T_{n}\right) \cap \operatorname{GMEP}(\Theta, \varphi, A) \cap$ $\operatorname{SGEP}(G) \cap I\left(B_{2}, R_{2}\right) \cap I\left(B_{1}, R_{1}\right) \neq \emptyset$ where $G$ is defined as in Proposition CY. Let $\left\{\alpha_{n}\right\},\left\{\beta_{n}\right\}$ and $\left\{\delta_{n}\right\}$ be three sequences in $[0,1]$. Assume that:

(i) $\mathrm{K}: H \rightarrow \mathbf{R}$ is strongly convex with constant $\sigma>0$ and its derivative $K^{\prime}$ is Lipschitz continuous with constant $v>0$ such that the function $x \mapsto\left\langle y-x, K^{\prime}(x)\right\rangle$ is weakly upper semicontinuous for each $y \in H$;

(ii) for each $x \in H$, there exist a bounded subset $D_{x} \subset C$ and $z_{x} \in C$ such that for any $y \notin D_{x}$,

$$
\begin{aligned}
& \Theta\left(y, z_{x}\right)+\varphi\left(z_{x}\right)-\varphi(y) \\
& \quad+\frac{1}{r}\left\langle K^{\prime}(y)-K^{\prime}(x), z_{x}-y\right\rangle<0
\end{aligned}
$$

(iii) $\lim _{n \rightarrow \infty} \alpha_{n}=0, \sum_{n=1}^{\infty} \alpha_{n}=\infty, 0<\liminf _{n \rightarrow \infty} \beta_{n} \leq$ $\limsup _{n \rightarrow \infty} \beta_{n}<1$ and $0<\liminf _{n \rightarrow \infty} \delta_{n} \leq$ $\lim \sup _{n \rightarrow \infty} \delta_{n}<1$;

(iv) $\nu_{k} \in\left(0,2 \zeta_{k}\right), \quad k=1,2, \quad \mu_{i} \in\left(0,2 \eta_{i}\right), i=1,2$, and $\left\{r_{n}\right\} \subset[0,2 \zeta]$ satisfies $0<\liminf _{n \rightarrow \infty} r_{n} \leq \limsup _{n \rightarrow \infty} r_{n}<$ $2 \zeta$;

(v) $\lim _{n \rightarrow \infty}\left(\left|\delta_{n+1}-\delta_{n}\right|+\left|r_{n+1}-r_{n}\right|\right)=0$.

Given $x_{1} \in H$ arbitrarily, then the sequence $\left\{x_{n}\right\}$ generated iteratively by

$$
\begin{gathered}
u_{n}=S_{r_{n}}^{(\Theta, \varphi)}\left(I-r_{n} A\right) x_{n}, \\
z_{n}=J_{R_{2}, \mu_{2}}\left(I-\mu_{2} B_{2}\right) J_{R_{1}, \mu_{1}}\left(I-\mu_{1} B_{1}\right) u_{n}, \\
y_{n}=\delta_{n} G z_{n}+\left(1-\delta_{n}\right) W_{n} z_{n}, \\
x_{n+1}=\alpha_{n}\left(u+\gamma f\left(x_{n}\right)\right)+\beta_{n} x_{n} \\
+\left(\left(1-\beta_{n}\right) I-\alpha_{n}(I+\mu V)\right) W_{n} y_{n}, \quad \forall n \geq 1,
\end{gathered}
$$

converges strongly to $x^{*} \in \Omega$ which solves the following optimization problem provided $S_{r}^{(\Theta, \varphi)}$ is firmly nonexpansive:

$$
\min _{x \in \Omega} \frac{\mu}{2}\langle A x, x\rangle+\frac{1}{2}\|x-u\|^{2}-h(x),
$$

where $h$ is the potential function of $\gamma f$. 
Corollary 24. Let $C$ be a nonempty closed convex subset of a real Hilbert space $H$. Let $\Theta, \Theta_{1}, \Theta_{2}$ be three bifunctions from $\mathrm{C} \times \mathrm{C}$ to $\mathbf{R}$ satisfying (H1)-(H4) and $\varphi: C \rightarrow \mathbf{R}$ be a lower semicontinuous and convex functional. Let $R: C \rightarrow 2^{H}$ be a maximal monotone mapping and let $A, A_{k}: H \rightarrow H$ and $B$ : $C \rightarrow H$ be $\zeta$-inverse strongly monotone, $\zeta_{k}$-inverse strongly monotone and $\eta$-inverse strongly monotone, respectively, for $k=1,2$. Let $\left\{T_{n}\right\}_{n=1}^{\infty}$ be a sequence of nonexpansive mappings on $H$ and $\left\{\lambda_{n}\right\}$ be a sequence in $(0, b]$ for some $b \in(0,1)$. Let $V$ be a $\bar{\gamma}$-strongly positive bounded linear operator and $f: H \rightarrow H$ be an l-Lipschitzian mapping with $\gamma l<(1+\mu) \bar{\gamma}$. Let $W_{n}$ be the $W$-mapping defined by (9). Assume that $\Omega:=$ $\cap_{n=1}^{\infty} \operatorname{Fix}\left(T_{n}\right) \cap \operatorname{GMEP}(\Theta, \varphi, A) \cap \operatorname{SGEP}(G) \cap I(B, R) \neq \emptyset$ where $G$ is defined as in Proposition CY. Let $\left\{\alpha_{n}\right\},\left\{\beta_{n}\right\}$ and $\left\{\delta_{n}\right\}$ be three sequences in $[0,1]$. Assume that:

(i) $K: H \rightarrow \mathbf{R}$ is strongly convex with constant $\sigma>0$ and its derivative $K^{\prime}$ is Lipschitz continuous with constant $v>0$ such that the function $x \mapsto\left\langle y-x, K^{\prime}(x)\right\rangle$ is weakly upper semicontinuous for each $y \in H$;

(ii) for each $x \in H$, there exist a bounded subset $D_{x} \subset C$ and $z_{x} \in C$ such that for any $y \notin D_{x}$,

$$
\begin{aligned}
& \Theta\left(y, z_{x}\right)+\varphi\left(z_{x}\right)-\varphi(y) \\
& +\frac{1}{r}\left\langle K^{\prime}(y)-K^{\prime}(x), z_{x}-y\right\rangle<0 ;
\end{aligned}
$$

(iii) $\lim _{n \rightarrow \infty} \alpha_{n}=0, \sum_{n=1}^{\infty} \alpha_{n}=\infty, 0<\liminf _{n \rightarrow \infty} \beta_{n} \leq$ $\lim \sup _{n \rightarrow \infty} \beta_{n}<1$ and $0<\liminf _{n \rightarrow \infty} \delta_{n} \leq$ $\lim \sup _{n \rightarrow \infty} \delta_{n}<1$

(iv) $v_{k} \in\left(0,2 \zeta_{k}\right), k=1,2, \lambda \in(0,2 \eta)$, and $\left\{r_{n}\right\} \subset[0,2 \zeta]$ satisfies $0<\liminf _{n \rightarrow \infty} r_{n} \leq \limsup _{n \rightarrow \infty} r_{n}<2 \zeta$;

(v) $\lim _{n \rightarrow \infty}\left(\left|\delta_{n+1}-\delta_{n}\right|+\left|r_{n+1}-r_{n}\right|\right)=0$.

Given $x_{1} \in H$ arbitrarily, then the sequence $\left\{x_{n}\right\}$ generated iteratively by

$$
\begin{gathered}
u_{n}=S_{r_{n}}^{(\Theta, \varphi)}\left(I-r_{n} A\right) x_{n}, \\
y_{n}=\delta_{n} G J_{R, \lambda}(I-\lambda B) u_{n} \\
+\left(1-\delta_{n}\right) W_{n} J_{R, \lambda}(I-\lambda B) u_{n}, \\
x_{n+1}=\alpha_{n}\left(u+\gamma f\left(x_{n}\right)\right)+\beta_{n} x_{n} \\
+\left(\left(1-\beta_{n}\right) I-\alpha_{n}(I+\mu V)\right) W_{n} y_{n}, \quad \forall n \geq 1,
\end{gathered}
$$

converges strongly to $x^{*} \in \Omega$ which solves the following optimization problem provided $S_{r}^{(\Theta, \varphi)}$ is firmly nonexpansive:

$$
\min _{x \in \Omega} \frac{\mu}{2}\langle A x, x\rangle+\frac{1}{2}\|x-u\|^{2}-h(x),
$$

where $h$ is the potential function of $\gamma f$.

Corollary 25. Let $C$ be a nonempty closed convex subset of a real Hilbert space $H$. Let $N$ be an integer. Let $\Theta, \Theta_{1}, \Theta_{2}$ be three bifunctions from $\mathrm{C} \times \mathrm{C}$ to $\mathbf{R}$ satisfying (H1)-(H4) and $\varphi: C \rightarrow$ $\mathbf{R}$ be a lower semicontinuous and convex functional. Let $R_{i}$ : $C \rightarrow 2^{H}$ be a maximal monotone mapping and let $A_{k}: H \rightarrow$ $H$ and $B_{i}: C \rightarrow H$ be $\zeta_{k}$-inverse strongly monotone and $\eta_{i^{-}}$ inverse strongly monotone, respectively, where $k \in\{1,2\}$ and $i \in\{1,2, \ldots, N\}$. Let $\left\{T_{n}\right\}_{n=1}^{\infty}$ be a sequence of nonexpansive mappings on $H$ and $\left\{\lambda_{n}\right\}$ be a sequence in $(0, b]$ for some $b \in$ $(0,1)$. Let $V$ be a $\bar{\gamma}$-strongly positive bounded linear operator and $f: H \rightarrow H$ be an l-Lipschitzian mapping with $\gamma l<$ $(1+\mu) \bar{\gamma}$. Let $W_{n}$ be the $W$-mapping defined by (9). Assume that $\Omega:=\cap_{n=1}^{\infty} \operatorname{Fix}\left(T_{n}\right) \cap \operatorname{MEP}(\Theta, \varphi) \cap \operatorname{SGEP}(G) \cap \cap_{i=1}^{N} I\left(B_{i}, R_{i}\right) \neq \emptyset$ where $G$ is defined as in Proposition CY. Let $\left\{\alpha_{n}\right\},\left\{\beta_{n}\right\}$ and $\left\{\delta_{n}\right\}$ be three sequences in $[0,1]$. Assume that:

(i) $K: H \rightarrow \mathbf{R}$ is strongly convex with constant $\sigma>0$ and its derivative $K^{\prime}$ is Lipschitz continuous with constant $v>0$ such that the function $x \mapsto\left\langle y-x, K^{\prime}(x)\right\rangle$ is weakly upper semicontinuous for each $y \in H$;

(ii) for each $x \in H$, there exist a bounded subset $D_{x} \subset C$ and $z_{x} \in C$ such that for any $y \notin D_{x}$,

$$
\begin{aligned}
& \Theta\left(y, z_{x}\right)+\varphi\left(z_{x}\right)-\varphi(y) \\
& +\frac{1}{r}\left\langle K^{\prime}(y)-K^{\prime}(x), z_{x}-y\right\rangle<0 ;
\end{aligned}
$$

(iii) $\lim _{n \rightarrow \infty} \alpha_{n}=0, \sum_{n=1}^{\infty} \alpha_{n}=\infty, 0<\liminf _{n \rightarrow \infty} \beta_{n} \leq$ $\lim \sup _{n \rightarrow \infty} \beta_{n}<1$ and $0<\liminf _{n \rightarrow \infty} \delta_{n} \leq$ $\lim \sup _{n \rightarrow \infty} \delta_{n}<1$;

(iv) $v_{k} \in\left(0,2 \zeta_{k}\right), k \in\{1,2\}, \mu_{i} \in\left(0,2 \eta_{i}\right), i \in$ $\{1,2, \ldots, N\}$, and $\left\{r_{n}\right\}$ is a bounded sequence in $(0, \infty)$ satisfying lim inf ${ }_{n \rightarrow \infty} r_{n}>0$;

(v) $\lim _{n \rightarrow \infty}\left(\left|\delta_{n+1}-\delta_{n}\right|+\left|r_{n+1}-r_{n}\right|\right)=0$.

Given $x_{1} \in H$ arbitrarily, then the sequence $\left\{x_{n}\right\}$ generated iteratively by

$$
\begin{aligned}
\Theta\left(u_{n}, y\right)+ & \varphi(y)-\varphi\left(u_{n}\right)+\frac{1}{r_{n}}\left\langle k^{\prime}\left(u_{n}\right)-k^{\prime}\left(x_{n}\right), y-u_{n}\right\rangle \\
\geq 0, \quad \forall y \in C, & \\
z_{n}= & J_{R_{N}, \mu_{N}}\left(I-\mu_{N} B_{N}\right) J_{R_{N-1}, \mu_{N-1}} \\
& \times\left(I-\mu_{N-1} B_{N-1}\right) \cdots J_{R_{1}, \mu_{1}}\left(I-\mu_{1} B_{1}\right) u_{n}, \\
& y_{n}=\delta_{n} G z_{n}+\left(1-\delta_{n}\right) W_{n} z_{n}, \\
x_{n+1}= & \alpha_{n}\left(u+\gamma f\left(x_{n}\right)\right)+\beta_{n} x_{n} \\
& +\left(\left(1-\beta_{n}\right) I-\alpha_{n}(I+\mu V)\right) W_{n} y_{n}, \quad \forall n \geq 1,
\end{aligned}
$$

converges strongly to $x^{*} \in \Omega$ which solves the following optimization problem provided $S_{r}^{(\Theta, \varphi)}$ is firmly nonexpansive:

$$
\min _{x \in \Omega} \frac{\mu}{2}\langle A x, x\rangle+\frac{1}{2}\|x-u\|^{2}-h(x),
$$

where $h$ is the potential function of $\gamma f$.

Proof. In Theorem 21, for all $n \geq 1, u_{n}=S_{r_{n}}^{(\Theta, \varphi)}\left(I-r_{n} A\right) x_{n}$ is equivalent to

$$
\begin{aligned}
& \Theta\left(u_{n}, y\right)+\varphi(y)-\varphi\left(u_{n}\right)+\left\langle A x_{n}, y-u_{n}\right\rangle \\
& +\frac{1}{r_{n}}\left\langle k^{\prime}\left(u_{n}\right)-k^{\prime}\left(x_{n}\right), y-u_{n}\right\rangle \geq 0, \quad \forall y \in C .
\end{aligned}
$$


Put $A \equiv 0$. Then it follows that

$$
\begin{aligned}
& \Theta\left(u_{n}, y\right)+\varphi(y)-\varphi\left(u_{n}\right)+\frac{1}{r_{n}}\left\langle k^{\prime}\left(u_{n}\right)-k^{\prime}\left(x_{n}\right), y-u_{n}\right\rangle \\
& \geq 0, \quad \forall y \in C .
\end{aligned}
$$

Observe that for all $\zeta \in(0, \infty)$

$$
\langle A x-A y, x-y\rangle \geq \zeta\|A x-A y\|^{2}, \quad \forall x, y \in H .
$$

So, whenever $0<\liminf _{n \rightarrow \infty} r_{n} \leq \limsup _{n \rightarrow \infty} r_{n}<2 \zeta$ for some $\zeta \in(0, \infty)$, we obtain the desired result by using Theorem 21.

Let $T: H \rightarrow H$ be a $\kappa$-strictly pseudocontractive mapping. For recent convergence result for strictly pseudocontractive mappings, we refer to [16]. Putting $A=I-T$, we know that for all $x, y \in H$

$$
\|(I-A) x-(I-A) y\|^{2} \leq\|x-y\|^{2}+\kappa\|A x-A y\|^{2} .
$$

Note that

$$
\begin{aligned}
\|(I-A) x-(I-A) y\|^{2}= & \|x-y\|^{2}+\|A x-A y\|^{2} \\
& -2\langle A x-A y, x-y\rangle .
\end{aligned}
$$

Hence we have for all $x, y \in H$

$$
\langle A x-A y, x-y\rangle \geq \frac{1-\kappa}{2}\|A x-A y\|^{2} .
$$

Consequently, if $T: H \rightarrow H$ is a $\kappa$-strictly pseudocontractive mapping, then the mapping $A=I-T$ is $(1-\kappa) / 2$-inverse strongly monotone.

Corollary 26. Let $C$ be a nonempty closed convex subset of a real Hilbert space $H$. Let $N$ be an integer. Let $\Theta, \Theta_{1}, \Theta_{2}$ be three bifunctions from $\mathrm{C} \times \mathrm{C}$ to $\mathbf{R}$ satisfying $(\mathrm{H1})-(\mathrm{H} 4)$ and $\varphi$ : $C \rightarrow \mathbf{R}$ be a lower semicontinuous and convex functional. Let $R_{i}: C \rightarrow 2^{H}$ be a maximal monotone mapping and let $T, A_{k}$ : $H \rightarrow H$ and $B_{i}: C \rightarrow H$ be $\kappa$-strictly pseudocontractive, $\zeta_{k^{-}}$ inverse strongly monotone and $\eta_{i}$-inverse strongly monotone, respectively, where $k \in\{1,2\}$ and $i \in\{1,2, \ldots, N\}$. Let $\left\{T_{n}\right\}_{n=1}^{\infty}$ be a sequence of nonexpansive mappings on $H$ and $\left\{\lambda_{n}\right\}$ be a sequence in $(0, b]$ for some $b \in(0,1)$. Let $V$ be a $\bar{\gamma}$-strongly positive bounded linear operator and $f: H \rightarrow H$ be an lLipschitzian mapping with $\gamma l<(1+\mu) \bar{\gamma}$. Let $W_{n}$ be the $W$ mapping defined by (9). Assume that $\Omega:=\cap_{n=1}^{\infty} \operatorname{Fix}\left(T_{n}\right) \cap$ $\operatorname{GMEP}(\Theta, \varphi, A) \cap \operatorname{SGEP}(G) \cap \cap_{i=1}^{N} I\left(B_{i}, R_{i}\right) \neq \emptyset$ where $A=I-T$ and $G$ is defined as in Proposition CY. Let $\left\{\alpha_{n}\right\},\left\{\beta_{n}\right\}$ and $\left\{\delta_{n}\right\}$ be three sequences in $[0,1]$. Assume that:

(i) $K: H \rightarrow \mathbf{R}$ is strongly convex with constant $\sigma>0$ and its derivative $K^{\prime}$ is Lipschitz continuous with constant $v>0$ such that the function $x \mapsto\left\langle y-x, K^{\prime}(x)\right\rangle$ is weakly upper semicontinuous for each $y \in H$;

(ii) for each $x \in H$, there exist a bounded subset $D_{x} \subset C$ and $z_{x} \in C$ such that for any $y \notin D_{x}$,

$$
\begin{aligned}
& \Theta\left(y, z_{x}\right)+\varphi\left(z_{x}\right)-\varphi(y) \\
& \quad+\frac{1}{r}\left\langle K^{\prime}(y)-K^{\prime}(x), z_{x}-y\right\rangle<0 ;
\end{aligned}
$$

(iii) $\lim _{n \rightarrow \infty} \alpha_{n}=0, \sum_{n=1}^{\infty} \alpha_{n}=\infty, 0<\liminf _{n \rightarrow \infty} \beta_{n} \leq$ $\limsup \operatorname{sum}_{n \rightarrow \infty} \beta_{n}<1$ and $0<\liminf _{n \rightarrow \infty} \delta_{n} \leq$ $\lim \sup _{n \rightarrow \infty} \delta_{n}<1$;

(iv) $v_{k} \in\left(0,2 \zeta_{k}\right), k \in\{1,2\}, \mu_{i} \in\left(0,2 \eta_{i}\right), i \in$ $\{1,2, \ldots, N\}$, and $\left\{r_{n}\right\} \subset[0,1-\kappa]$ satisfies

$$
0<\liminf _{n \rightarrow \infty} r_{n} \leq \limsup _{n \rightarrow \infty} r_{n}<1-\kappa ;
$$

(v) $\lim _{n \rightarrow \infty}\left(\left|\delta_{n+1}-\delta_{n}\right|+\left|r_{n+1}-r_{n}\right|\right)=0$.

Given $x_{1} \in H$ arbitrarily, then the sequence $\left\{x_{n}\right\}$ generated iteratively by

$$
\begin{gathered}
u_{n}=S_{r_{n}}^{(\Theta, \varphi)}\left(\left(1-r_{n}\right) x_{n}+r_{n} T x_{n}\right), \\
z_{n}=J_{R_{N}, \mu_{N}}\left(I-\mu_{N} B_{N}\right) J_{R_{N-1}, \mu_{N-1}} \\
\times\left(I-\mu_{N-1} B_{N-1}\right) \cdots J_{R_{1}, \mu_{1}}\left(I-\mu_{1} B_{1}\right) u_{n}, \\
y_{n}=\delta_{n} G z_{n}+\left(1-\delta_{n}\right) W_{n} z_{n}, \\
x_{n+1}=\alpha_{n}\left(u+\gamma f\left(x_{n}\right)\right)+\beta_{n} x_{n} \\
+\left(\left(1-\beta_{n}\right) I-\alpha_{n}(I+\mu V)\right) W_{n} y_{n}, \quad \forall n \geq 1,
\end{gathered}
$$

converges strongly to $x^{*} \in \Omega$ which solves the following optimization problem provided $S_{r}^{(\Theta, \varphi)}$ is firmly nonexpansive:

$$
\min _{x \in \Omega} \frac{\mu}{2}\langle A x, x\rangle+\frac{1}{2}\|x-u\|^{2}-h(x),
$$

where $h$ is the potential function of $\gamma f$.

Proof. Since $T$ is a $\kappa$-strictly pseudocontractive mapping, the mapping $A=I-T$ is $(1-\kappa) / 2$-inverse strongly monotone. In this case, put $\zeta=(1-\kappa) / 2$. Moreover, we obtain that

$$
\begin{aligned}
u_{n} & =S_{r_{n}}^{(\Theta, \varphi)}\left(I-r_{n} A\right) x_{n} \\
& =S_{r_{n}}^{(\Theta, \varphi)}\left(x_{n}-r_{n}(I-T) x_{n}\right) \\
& =S_{r_{n}}^{(\Theta, \varphi)}\left(\left(1-r_{n}\right) x_{n}+r_{n} T x_{n}\right) .
\end{aligned}
$$

So, from Theorem 21, we obtain the desired result.

Corollary 27. Let $C$ be a nonempty closed convex subset of a real Hilbert space $H$. Let $N$ be an integer. Let $\Theta, \Theta_{1}, \Theta_{2}$ be three bifunctions from $\mathrm{C} \times \mathrm{C}$ to $\mathbf{R}$ satisfying $(\mathrm{H1})-(\mathrm{H} 4)$ and $\varphi$ : $C \rightarrow \mathbf{R}$ be a lower semicontinuous and convex functional. Let $R_{i}: C \rightarrow 2^{H}$ be a maximal monotone mapping and let $A, A_{k}$ : $H \rightarrow H$ and $B_{i}: C \rightarrow H$ be $\zeta$-inverse strongly monotone, $\zeta_{k^{-}}$ inverse strongly monotone and $\eta_{i}$-inverse strongly monotone, respectively, where $k \in\{1,2\}$ and $i \in\{1,2, \ldots, N\}$. Let $V$ be a $\bar{\gamma}$-strongly positive bounded linear operator and $f: H \rightarrow H$ be an l-Lipschitzian mapping with $\gamma l<(1+\mu) \bar{\gamma}$. Assume that $\Omega:=\operatorname{GMEP}(\Theta, \varphi, A) \cap \operatorname{SGEP}(G) \cap \cap_{i=1}^{N} I\left(B_{i}, R_{i}\right) \neq \emptyset$ where $G$ is defined as in Proposition CY. Let $\left\{\alpha_{n}\right\},\left\{\beta_{n}\right\}$ and $\left\{\delta_{n}\right\}$ be three sequences in $[0,1]$. Assume that:

(i) $\mathrm{K}: H \rightarrow \mathbf{R}$ is strongly convex with constant $\sigma>0$ and its derivative $K^{\prime}$ is Lipschitz continuous with constant $v>0$ such that the function $x \mapsto\left\langle y-x, K^{\prime}(x)\right\rangle$ is weakly upper semicontinuous for each $y \in H$; 
(ii) for each $x \in H$, there exist a bounded subset $D_{x} \subset C$ and $z_{x} \in C$ such that for any $y \notin D_{x}$,

$$
\begin{aligned}
& \Theta\left(y, z_{x}\right)+\varphi\left(z_{x}\right)-\varphi(y) \\
& +\frac{1}{r}\left\langle K^{\prime}(y)-K^{\prime}(x), z_{x}-y\right\rangle<0 ;
\end{aligned}
$$

(iii) $\lim _{n \rightarrow \infty} \alpha_{n}=0, \sum_{n=1}^{\infty} \alpha_{n}=\infty, 0<\liminf _{n \rightarrow \infty} \beta_{n} \leq$ $\limsup \sup _{n \rightarrow \infty} \beta_{n}<1$ and $0<\liminf _{n \rightarrow \infty} \delta_{n} \leq$ $\lim \sup _{n \rightarrow \infty} \delta_{n}<1$;

(iv) $v_{k} \in\left(0,2 \zeta_{k}\right), k \in\{1,2\}, \mu_{i} \in\left(0,2 \eta_{i}\right), i \in$ $\{1,2, \ldots, N\}$, and $\left\{r_{n}\right\} \subset[0,2 \zeta]$ satisfies

$$
0<\liminf _{n \rightarrow \infty} r_{n} \leq \limsup _{n \rightarrow \infty} r_{n}<2 \zeta
$$

(v) $\lim _{n \rightarrow \infty}\left(\left|\delta_{n+1}-\delta_{n}\right|+\left|r_{n+1}-r_{n}\right|\right)=0$.

Given $x_{1} \in H$ arbitrarily, then the sequence $\left\{x_{n}\right\}$ generated iteratively by

$$
\begin{gathered}
u_{n}=S_{r_{n}}^{(\Theta, \varphi)}\left(I-r_{n} A\right) x_{n}, \\
z_{n}=J_{R_{N}, \mu_{N}}\left(I-\mu_{N} B_{N}\right) J_{R_{N-1}, \mu_{N-1}} \\
\times\left(I-\mu_{N-1} B_{N-1}\right) \cdots J_{R_{1}, \mu_{1}}\left(I-\mu_{1} B_{1}\right) u_{n}, \\
y_{n}=\delta_{n} G z_{n}+\left(1-\delta_{n}\right) z_{n}, \\
x_{n+1}=\alpha_{n}\left(u+\gamma f\left(x_{n}\right)\right)+\beta_{n} x_{n} \\
+\left(\left(1-\beta_{n}\right) I-\alpha_{n}(I+\mu V)\right) y_{n}, \quad \forall n \geq 1,
\end{gathered}
$$

converges strongly to $x^{*} \in \Omega$ which solves the following optimization problem provided $S_{r}^{(\Theta, \varphi)}$ is firmly nonexpansive:

$$
\min _{x \in \Omega} \frac{\mu}{2}\langle A x, x\rangle+\frac{1}{2}\|x-u\|^{2}-h(x),
$$

where $h$ is the potential function of $\gamma f$.

Proof. Put $T_{n} x=x$ for all integers $n \geq 1$ and all $x \in H$. Then, the desired result follows from Theorem 21 .

Corollary 28. Let $C$ be a nonempty closed convex subset of a real Hilbert space $H$. Let $N$ be an integer. Let $\Theta_{1}$ and $\Theta_{2}$ be two bifunctions from $\mathrm{C} \times \mathrm{C}$ to $\mathbf{R}$ satisfying $(\mathrm{H1})-(\mathrm{H} 4)$. Let $\mathrm{R}_{i}: \mathrm{C} \rightarrow$ $2^{H}$ be a maximal monotone mapping and let $A_{k}: H \rightarrow H$ and $B_{i}: C \rightarrow H$ be $\zeta_{k}$-inverse strongly monotone and $\eta_{i^{-}}$ inverse strongly monotone, respectively, where $k \in\{1,2\}$ and $i \in\{1,2, \ldots, N\}$. Let $\left\{T_{n}\right\}_{n=1}^{\infty}$ be a sequence of nonexpansive mappings on $H$ and $\left\{\lambda_{n}\right\}$ be a sequence in $(0, b]$ for some $b \in$ $(0,1)$. Let $V$ be a $\bar{\gamma}$-strongly positive bounded linear operator and $f: H \rightarrow H$ be an l-Lipschitzian mapping with $\gamma l<$ $(1+\mu) \bar{\gamma}$. Let $W_{n}$ be the $W$-mapping defined by (9). Assume that $\Omega:=\cap_{n=1}^{\infty} \operatorname{Fix}\left(T_{n}\right) \cap \operatorname{SGEP}(G) \cap \cap_{i=1}^{N} I\left(B_{i}, R_{i}\right) \neq \emptyset$ where $G$ is defined as in Proposition CY. Let $\left\{\alpha_{n}\right\},\left\{\beta_{n}\right\}$ and $\left\{\delta_{n}\right\}$ be three sequences in $[0,1]$. Assume that:

(i) $\lim _{n \rightarrow \infty} \alpha_{n}=0, \sum_{n=1}^{\infty} \alpha_{n}=\infty, 0<\liminf _{n \rightarrow \infty} \beta_{n} \leq$ $\limsup \sup _{n \rightarrow \infty} \beta_{n}<1$ and $0<\liminf _{n \rightarrow \infty} \delta_{n} \leq$ $\lim \sup _{n \rightarrow \infty} \delta_{n}<1$; (ii) $v_{k} \in\left(0,2 \zeta_{k}\right), k \in\{1,2\}, \mu_{i} \in\left(0,2 \eta_{i}\right), i \in\{1,2, \ldots, N\}$, and $\left\{r_{n}\right\} \subset[0,2 \zeta]$ satisfies

$$
0<\liminf _{n \rightarrow \infty} r_{n} \leq \limsup _{n \rightarrow \infty} r_{n}<2 \zeta ;
$$

(iii) $\lim _{n \rightarrow \infty}\left(\left|\delta_{n+1}-\delta_{n}\right|+\left|r_{n+1}-r_{n}\right|\right)=0$.

Given $x_{1} \in C$ arbitrarily, then the sequence $\left\{x_{n}\right\}$ generated iteratively by

$$
\begin{aligned}
z_{n}= & J_{R_{N}, \mu_{N}}\left(I-\mu_{N} B_{N}\right) J_{R_{N-1}, \mu_{N-1}} \\
& \times\left(I-\mu_{N-1} B_{N-1}\right) \cdots J_{R_{1}, \mu_{1}}\left(I-\mu_{1} B_{1}\right) x_{n}, \\
& y_{n}=\delta_{n} G z_{n}+\left(1-\delta_{n}\right) W_{n} z_{n}, \\
x_{n+1}= & \alpha_{n}\left(u+\gamma f\left(x_{n}\right)\right)+\beta_{n} x_{n} \\
+ & \left(\left(1-\beta_{n}\right) I-\alpha_{n}(I+\mu V)\right) W_{n} y_{n}, \quad \forall n \geq 1,
\end{aligned}
$$

converges strongly to $x^{*} \in \Omega$ which solves the following optimization problem provided $S_{r}^{(\Theta, \varphi)}$ is firmly nonexpansive:

$$
\min _{x \in \Omega} \frac{\mu}{2}\langle A x, x\rangle+\frac{1}{2}\|x-u\|^{2}-h(x),
$$

where $h$ is the potential function of $\gamma f$.

Proof. Put $\Theta(x, y)=0, \varphi(x)=0$ for all $x, y \in C, A x=0$ for all $x \in H$ and $r_{n}=1$. Take $K(x)=(1 / 2)\|x\|^{2}$ for all $x \in H$. Then we get $u_{n}=x_{n}$ in Theorem 21 and the conclusion follows.

\section{Conflict of Interests}

The authors declare that there is no conflict of interests regarding the publication of this paper.

\section{Acknowledgment}

This article was funded by the Deanship of Scientific Research (DSR), King Abdulaziz University, Jeddah. The authors, therefore, acknowledge with thanks DSR for the technical and financial support. This research was partially supported to first author by the National Science Foundation of China (11071169), Innovation Program of Shanghai Municipal Education Commission (09ZZ133) and Ph.D. Program Foundation of Ministry of Education of China (20123127110002). The authors are thankful to the reviewers for their valuable suggestions/comments with appreciation.

\section{References}

[1] J. L. Lions, Quelques Méthodes de Résolution des Problèmes Aux Limites non Linéaire, Dunod, Paris, France, 1969.

[2] J.-W. Peng and J.-C. Yao, "A new hybrid-extragradient method for generalized mixed equilibrium problems, fixed point problems and variational inequality problems," Taiwanese Journal of Mathematics, vol. 12, no. 6, pp. 1401-1432, 2008.

[3] Q. H. Ansari and J.-C. Yao, "Systems of generalized variational inequalities and their applications," Applicable Analysis, vol. 76, no. 3-4, pp. 203-217, 2000. 
[4] Q. H. Ansari, S. Schaible, and J.-C. Yao, "The system of generalized vector equilibrium problems with applications," Journal of Global Optimization, vol. 22, no. 1-4, pp. 3-16, 2002.

[5] L.-J. Lin and Q. H. Ansari, "Collective fixed points and maximal elements with applications to abstract economies," Journal of Mathematical Analysis and Applications, vol. 296, no. 2, pp. 455472, 2004.

[6] S. Al-Homidan, Q. H. Ansari, and S. Schaible, "Existence of solutions of systems of generalized implicit vector variational inequalities," Journal of Optimization Theory and Applications, vol. 134, no. 3, pp. 515-531, 2007.

[7] S. Al-Homidan, Q. H. Ansari, and J.-C. Yao, "Collectively fixed point and maximal element theorems in topological semilattice spaces," Applicable Analysis, vol. 90, no. 6, pp. 865-888, 2011.

[8] S. Al-Homidan and Q. H. Ansari, "Fixed point theorems on product topological semilattice spaces, generalized abstract economies and systems of generalized vector quasi-equilibrium problems," Taiwanese Journal of Mathematics, vol. 15, no. 1, pp. 307-330, 2011.

[9] G. Cai and S. Q. Bu, "Strong and weak convergence theorems for general mixed equilibrium problems and variational inequality problems and fixed point problems in Hilbert spaces," Journal of Computational and Applied Mathematics, vol. 247, pp. 34-52, 2013.

[10] L. C. Ceng, H.-Y. Hu, and M. M. Wong, "Strong and weak convergence theorems for generalized mixed equilibrium problem with perturbation and fixed pointed problem of infinitely many nonexpansive mappings," Taiwanese Journal of Mathematics, vol. 15, no. 3, pp. 1341-1367, 2011.

[11] L.-C. Ceng, Q. H. Ansari, and S. Schaible, "Hybrid extragradient-like methods for generalized mixed equilibrium problems, systems of generalized equilibrium problems and optimization problems," Journal of Global Optimization, vol. 53, no. 1, pp. 69-96, 2012.

[12] L.-C. Ceng, S.-M. Guu, and J.-C. Yao, "Hybrid iterative method for finding common solutions of generalized mixed equilibrium and fixed point problems," Fixed Point Theory and Applications, vol. 2012, article 92, 2012.

[13] L.-C. Ceng and A. Petruşel, "Relaxed extragradient-like method for general system of generalized mixed equilibria and fixed point problem," Taiwanese Journal of Mathematics, vol. 16, no. 2, pp. 445-478, 2012.

[14] Y. Yao, Y.-C. Liou, and J.-C. Yao, "New relaxed hybridextragradient method for fixed point problems, a general system of variational inequality problems and generalized mixed equilibrium problems," Optimization, vol. 60, no. 3, pp. 395-412, 2011.

[15] Y. Yao, Y. J. Cho, and Y.-C. Liou, "Algorithms of common solutions for variational inclusions, mixed equilibrium problems and fixed point problems," European Journal of Operational Research, vol. 212, no. 2, pp. 242-250, 2011.

[16] L.-C. Ceng, Q. H. Ansari, S. Schaible, and J.-C. Yao, "Iterative methods for generalized equilibrium problems, systems of general generalized equilibrium problems and fixed point problems for nonexpansive mappings in Hilbert spaces," Fixed Point Theory, vol. 12, no. 2, pp. 293-308, 2011.

[17] L.-C. Ceng and J.-C. Yao, "A relaxed extragradient-like method for a generalized mixed equilibrium problem, a general system of generalized equilibria and a fixed point problem," Nonlinear Analysis. Theory, Methods \& Applications, vol. 72, no. 3-4, pp. 1922-1937, 2010.
[18] L.-C. Ceng, C.-Y. Wang, and J.-C. Yao, "Strong convergence theorems by a relaxed extragradient method for a general system of variational inequalities," Mathematical Methods of Operations Research, vol. 67, no. 3, pp. 375-390, 2008.

[19] R. T. Rockafellar, "Monotone operators and the proximal point algorithm," SIAM Journal on Control and Optimization, vol. 14, no. 5, pp. 877-898, 1976.

[20] L.-C. Ceng and J.-C. Yao, "A hybrid iterative scheme for mixed equilibrium problems and fixed point problems," Journal of Computational and Applied Mathematics, vol. 214, no. 1, pp. 186201, 2008.

[21] J. G. O’Hara, P. Pillay, and H.-K. Xu, "Iterative approaches to convex feasibility problems in Banach spaces," Nonlinear Analysis. Theory, Methods \& Applications, vol. 64, no. 9, pp. 2022-2042, 2006.

[22] Y. Yao, Y.-C. Liou, and J.-C. Yao, "Convergence theorem for equilibrium problems and fixed point problems of infinite family of nonexpansive mappings," Fixed Point Theory and Applications, vol. 2007, Article ID 64363, 12 pages, 2007.

[23] K. Goebel and W. A. Kirk, Topics in Metric Fixed Point Theory, vol. 28, Cambridge University Press, Cambridge, UK, 1990.

[24] T. Suzuki, “Strong convergence of Krasnoselskii and Mann's type sequences for one-parameter nonexpansive semigroups without Bochner integrals," Journal of Mathematical Analysis and Applications, vol. 305, no. 1, pp. 227-239, 2005.

[25] H.-K. Xu, "Iterative algorithms for nonlinear operators," Journal of the London Mathematical Society, vol. 66, no. 1, pp. 240-256, 2002.

[26] N.-J. Huang, "A new completely general class of variational inclusions with noncompact valued mappings," Computers \& Mathematics with Applications, vol. 35, no. 10, pp. 9-14, 1998.

[27] L.-C. Zeng, S.-M. Guu, and J.-C. Yao, "Characterization of $H$-monotone operators with applications to variational inclusions," Computers \& Mathematics with Applications, vol. 50, no. 3-4, pp. 329-337, 2005.

[28] L.-C. Ceng, Q. H. Ansari, M. M. Wong, and J.-C. Yao, "Mann type hybrid extragradient method for variational inequalities, variational inclusions and fixed point problems," Fixed Point Theory, vol. 13, no. 2, pp. 403-422, 2012.

[29] Y. Yao, M. A. Noor, S. Zainab, and Y. C. Liou, "Mixed equilibrium problems and optimization problems," Journal of Mathematical Analysis and Applications, vol. 354, pp. 319-329, 2009. 


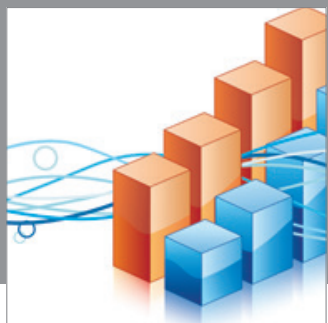

Advances in

Operations Research

mansans

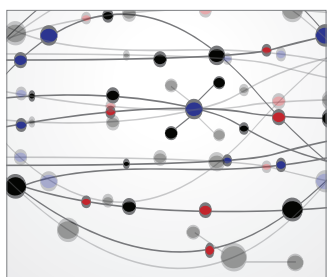

The Scientific World Journal
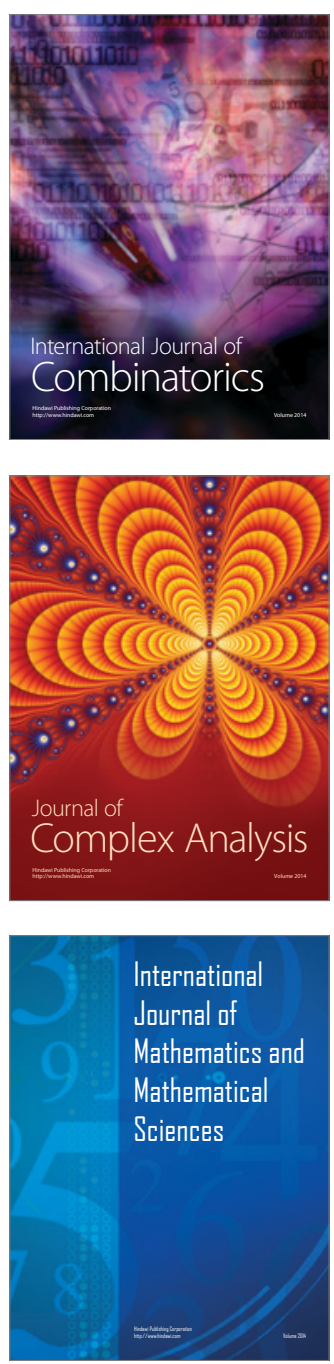
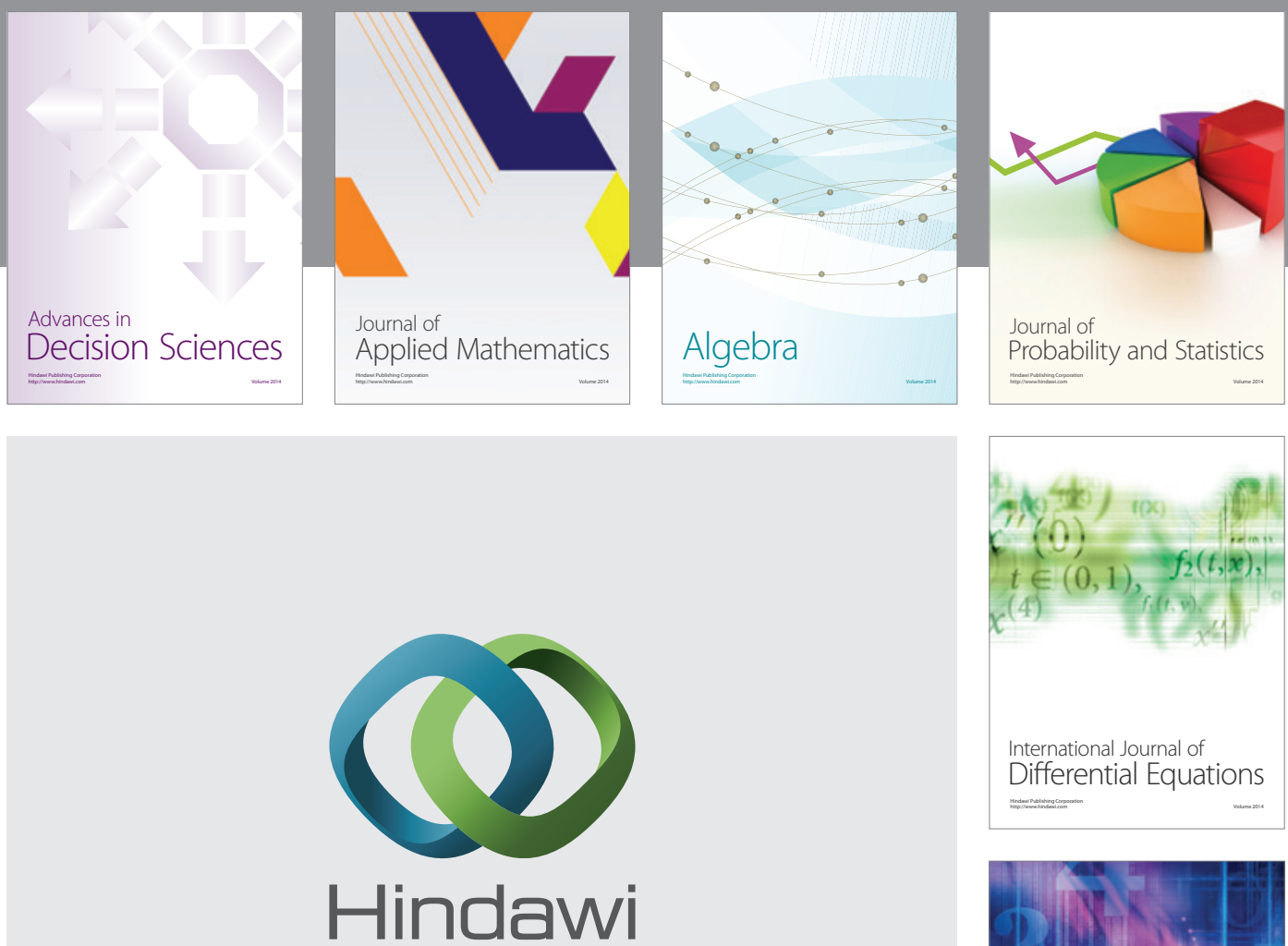

Submit your manuscripts at http://www.hindawi.com
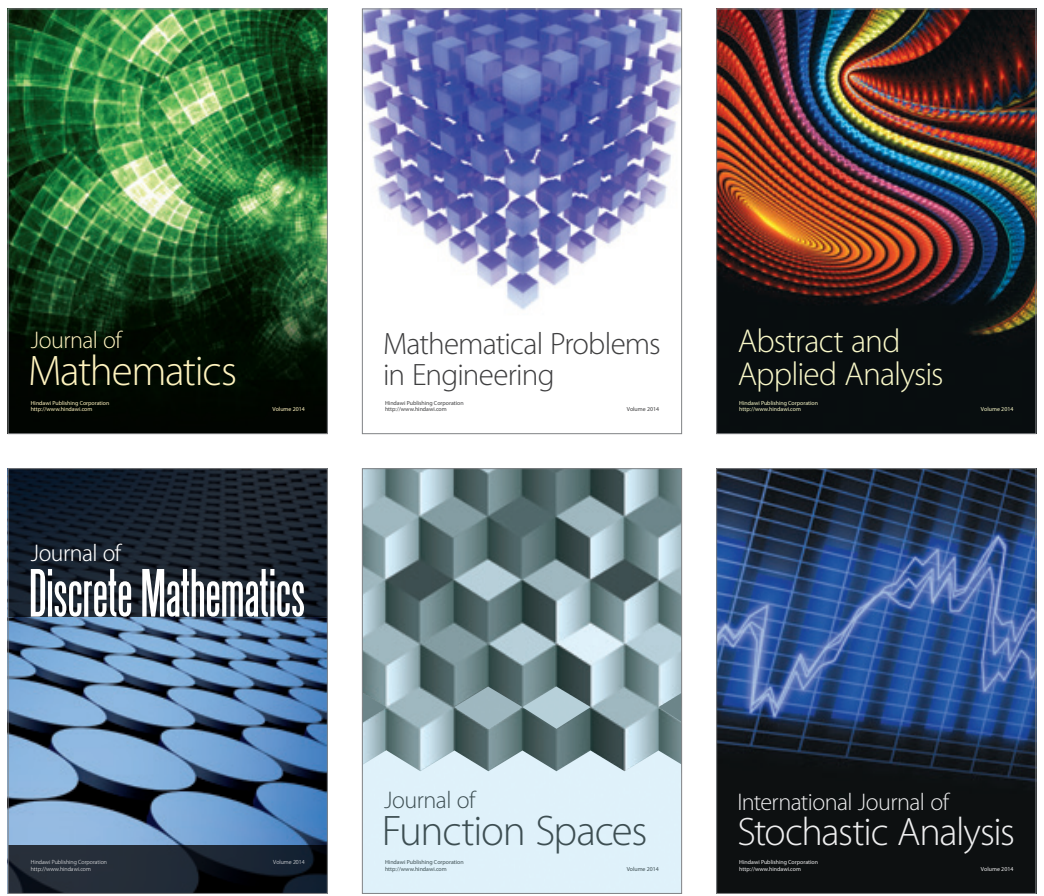

Journal of

Function Spaces

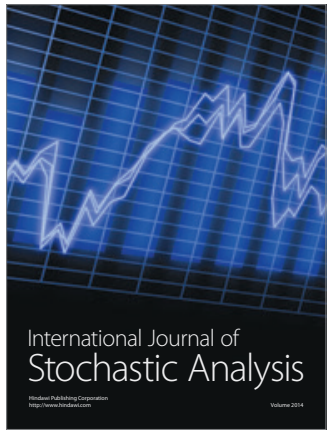

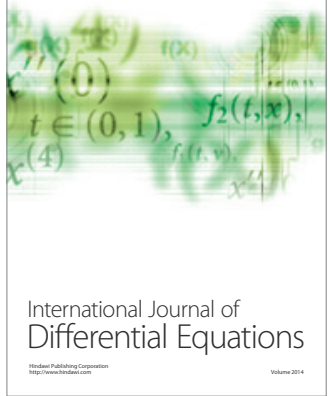
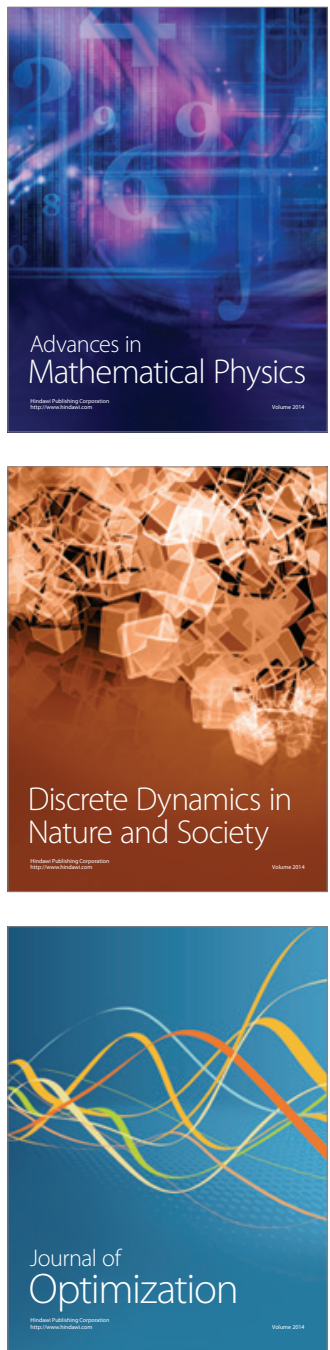